\title{
Identification of a novel member of the family Betaflexiviridae from the hallucinogenic plant Salvia divinorum
}

\author{
C. J. GOH, Y. HAHN ${ }^{*}$
}

Department of Life Science, Chung-Ang University, 84 Heukseok-ro, Dongjak-gu, Seoul 06974, Republic of Korea

Received April 1, 2019; accepted April 4, 2019

\begin{abstract}
Summary. - Betaflexiviridae is a family of plant-infecting RNA viruses with 11 recognized genera, of which genomes have diverse organization with three to six open reading frames (ORFs). A genome sequence of a novel Betaflexiviridae species, named Salvia divinorum RNA virus 1 (SdRV1), was identified in Salvia divinorum, herbal mint plant with hallucinogenic properties. The SdRV1 genome was predicted to have four ORFs encoding a replicase polyprotein (REP), a movement protein (MP), a coat protein (CP), and a putative nucleic acid-binding protein (NBP). Phylogenetic analyses based on the REP, MP, and CP sequences indicated that SdRV1 is most closely related to members of the genus Citrivirus. However, the genome organization of SdRV1 is the same as that of the genus Prunevirus. Moreover, the SdRV1 NBP had greatest sequence similarity with members of the genus Carlavirus. A complex evolutionary history involving ancestors of these three genera might have resulted in the unique phylogenetic position of SdRV1, which could be considered the founding member of a new genus in the family Betaflexiviridae. The genome sequence of SdRV1 might be useful for studies on the evolution of Betaflexiviridae.
\end{abstract}

Keywords: Salvia divinorum RNA virus 1; Betaflexiviridae; Salvia divinorum

\section{Introduction}

Betaflexiviridae is a family of plant-infecting viruses of the order Tymovirales, and consists of 11 approved genera: Capillovirus, Carlavirus, Chordovirus, Citrivirus, Divavirus, Foveavirus, Prunevirus, Robigovirus, Tepovirus, Trichovirus, and Vitivirus (Adams et al., 2012, 2016). Betaflexiviridae viruses have a flexuous particle and a positive-sense singlestranded RNA genome of approximately $6-9 \mathrm{~kb}$. The genome contains three to six open reading frames (ORFs) depending on the genus (Adams et al., 2012). The 11 genera can

"Corresponding author. E-mail: hahny@cau.ac.kr; phone: +82-2820-5812.

Abbreviations: $\mathrm{CLBV}=$ citrus leaf blotch virus; $\mathrm{CP}=$ coat protein; $\mathrm{MP}=$ movement protein; $\mathrm{NCBI}=$ National Center for Biotechnol ogy Information; NBP = nucleic acid-binding protein; $\mathrm{ORF}=$ open reading frame; $\mathrm{REP}=$ replicase polyprotein; $\mathrm{RdRp}=\mathrm{RNA}$-dependent RNA polymerase; SdRV1 = Salvia divinorum RNA virus 1; $\mathrm{TGB}=$ triple gene block be classified into five groups based on genome organization. The viruses of six of the genera (Capillovirus, Chordovirus, Citrivirus, Divavirus, Tepovirus, and Trichovirus) have three ORFs. ORF1 encodes a replicase polyprotein (REP), which has viral methyltransferase, 2OG-Fe(II) oxygenase superfamily, viral RNA helicase, and RNA-dependent RNA polymerase (RdRp) domains. ORF2 and ORF3 encode a movement protein (MP) and a coat protein $(\mathrm{CP})$, respectively (Adams et al., 2012; Rubino et al., 2012; Chavan et al., 2013; Marais et al., 2015). Viruses of the genus Prunevirus have four ORFs encoding REP, MP, CP, and a nucleic acidbinding protein (NBP) (Veerakone et al., 2018). Viruses of the genus Vitivirus have five ORFs encoding REP, a 20-K protein, MP, CP, and NBP (Martelli et al., 1997; Adams et al., 2012; Diaz-Lara et al., 2018). Viruses of the genera Foveavirus and Robigovirus have five ORFs encoding REP, three triple gene block proteins (TGB1, TGB2, and TGB3), and CP (Morozov and Solovyev, 2003; Prosser et al., 2015; Jo et al., 2017). Viruses of the genus Carlavirus have six ORFs encoding REP, TGB1, TGB2, TGB3, CP, and NBP (Adams et al., 2012; Li et al., 2013). 
Table 1. ORFs and functional domains of the SdRV1 genome

\begin{tabular}{|c|c|c|c|c|c|c|}
\hline ORF & nt position & nt length & aa length & Domain & Pfam & aa position \\
\hline \multirow[t]{4}{*}{ Replicase polyprotein } & $76-6219$ & 6144 & 2047 & Viral methyltransferase & PF01660 & $44-347$ \\
\hline & & & & 2OG-Fe(II) oxygenase superfamily & PF13532 & $939-1047$ \\
\hline & & & & Viral (superfamily 1) RNA helicase & PF01443 & $1249-1500$ \\
\hline & & & & RNA-dependent RNA polymerase & PF00978 & $1622-2024$ \\
\hline Movement protein & $6228-7253$ & 1026 & 341 & Viral movement protein & PF01107 & $14-196$ \\
\hline Coat protein & $7500-8591$ & 1092 & 363 & Viral coat protein & PF00286 & $169-315$ \\
\hline Putative nucleic acid-binding protein & $8705-9067$ & 363 & 120 & Viral nucleic acid-binding & PF05515 & $1-89$ \\
\hline
\end{tabular}

Salvia divinorum, which belongs to the Lamiaceae family, is a herbal mint plant with transient psychoactive properties. Owing to its hallucinogenic effects, for many centuries the Mazatec people of Oaxaca, Mexico, have ingested it as a tea or smoked it in traditional spiritual practices (Valdes, 1994; Chavkin et al., 2004). The hallucinatory compound found in S. divinorum is called salvinorin A; it has a highly selective and efficient agonistic effect on kappa-opioid receptors in humans (Roth et al., 2002; Chavkin et al., 2004). These receptors are located in parts of the central nervous system, and are known to be involved in psychiatric disorders such as anxiety, depression, and addiction (Butelman and Kreek, 2015; Anderson and Becker, 2017).

Salvinorin A from $S$. divinorum is considered a new medical substance for the treatment of mental disorders (Kivell et al., 2014; Riley et al., 2014). Several transcriptomic analyses using next-generation sequencing technology have been conducted to investigate the genetic features and biochemistry of S. divinorum (Chen et al., 2017; Pelot et al., 2017). Plant transcriptome data often contain sequences from RNA viruses that infect the plant samples, which may be identified as novel RNA viruses (Goh et al., 2018; Kim et al., 2018). In the present study, we analyzed a previously reported transcriptome dataset from the leaf trichomes of S. divinorum (Pelot et al., 2017), and identified a novel RNA virus of the family Betaflexiviridae.

\section{Materials and Methods}

Transcriptome dataset. We analyzed a previously reported plant transcriptome dataset isolated from the leaf trichomes of S. divinorum (6.8 gigabases of paired-end reads with a single run) (Pelot et al., 2017). The transcriptome dataset is available from the Sequence Read Archive (SRA) at the National Center for Biotechnology Information (NCBI; Acc. No. SRR3716680). Raw RNA-seq reads were filtered to collect high-quality reads using the Sickle program (version 1.33; https://github.com/najoshi/sickle) with the option “-q 30 -1 55." De novo assembly was conducted using the SPAdes Genome Assembler (version 3.10.1; http://cab.spbu.ru/software/ spades) with the "--rna" option (Bankevich et al., 2012).

Identification of viral genome contig. All the RNA contigs were examined to determine whether they contain a viral RdRp motif. The Pfam database (release 31.0; http://pfam.xfam.org) was parsed to collect reference sequences of viral RdRp motifs. Pfam Acc. Nos. with viral RdRp motifs include PF00602, PF00603, PF00604, PF00680, PF00946, PF00972, PF00978, PF00998, PF02123, PF03431, PF04196, PF04197, PF05788, PF05919, PF07925, PF08467, PF08716, PF08717, and PF12426. Representative viral RdRp motif sequences derived from 394 viruses were converted to a BLAST-searchable database. A BLASTX search was performed with the parameter "-evalue e-5" to compare the assembled contigs with the custom-built viral RdRp database. RNA-seq reads were mapped to a viral contig using the BWA program (version 0.7.16a-r1181;

\section{SdRV1 (9214 nt)}

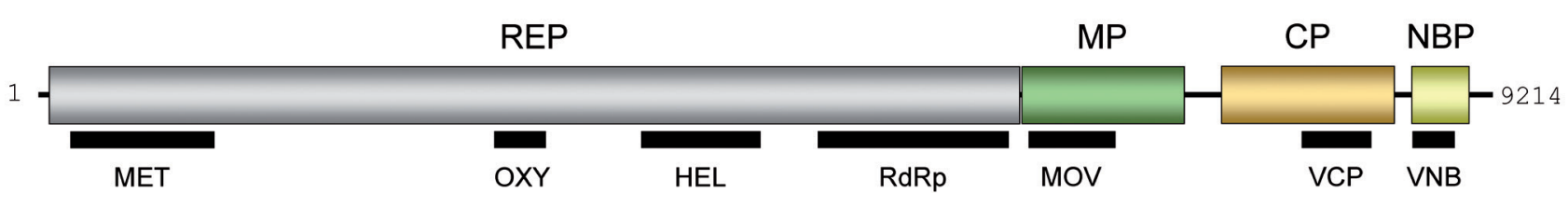

Fig. 1

Schematic representation of SdRV1 genome organization

Open reading frames (ORFs) are depicted as gray boxes: REP, replicase polyprotein; MP, movement protein; CP, coat protein; and NBP, putative nucleic acid-binding protein. Predicted Pfam domains are marked by lines below proteins: MET, viral methyltransferase; OXY, 2OG-Fe(II) oxygenase superfamily; HEL, viral RNA helicase; RdRp, RNA-dependent RNA polymerase; MOV, viral movement protein; VCP, viral coat protein; and VNB, viral nucleic acid-binding. The coordinates and lengths of ORFs and domains are presented in Table 1. 


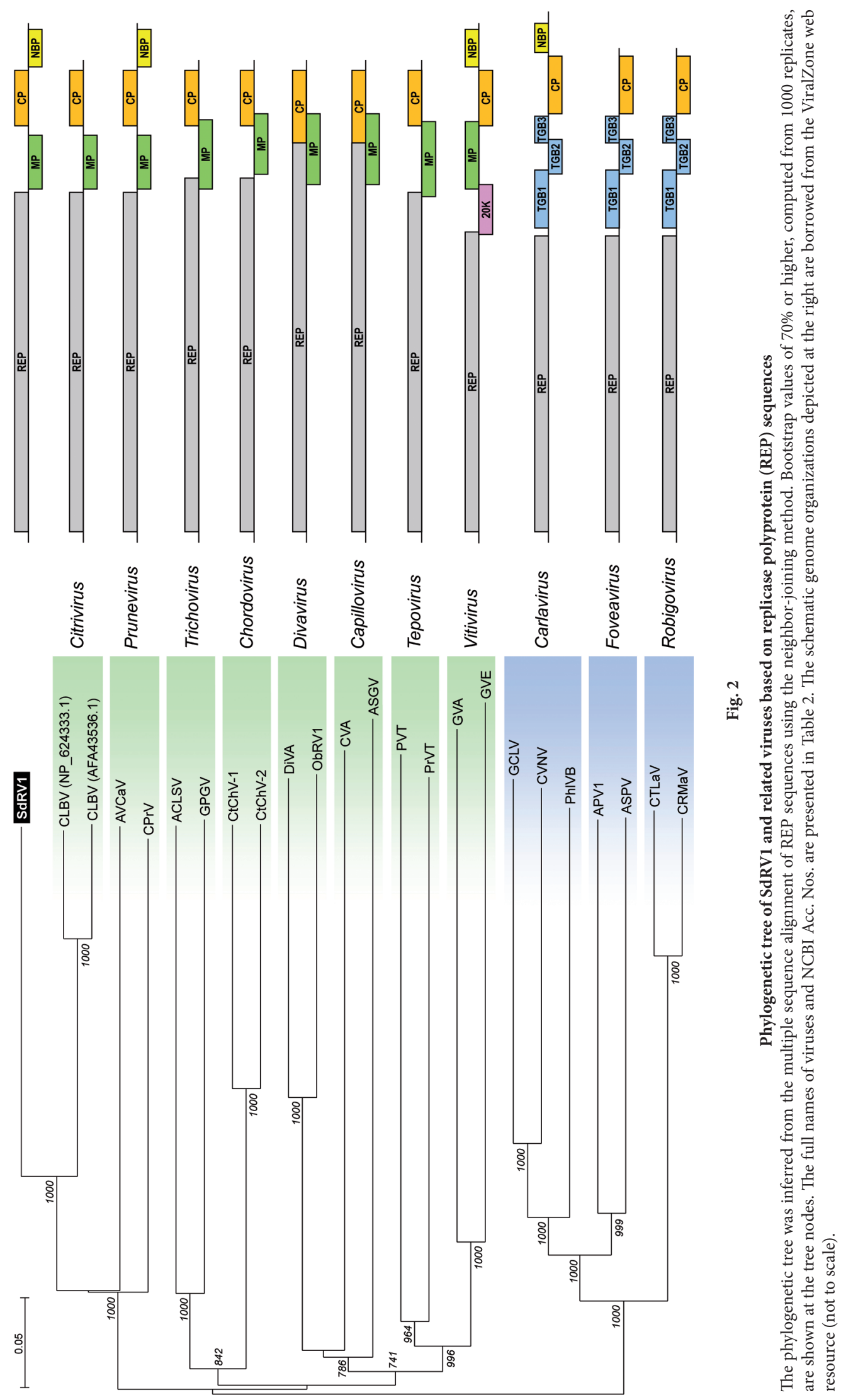


Table 2. Comparison of the sequence of replicase polyprotein (REP) from SdRV1 with REP sequences from representative members of Betaflexiviridae

\begin{tabular}{|c|c|c|c|c|c|c|c|}
\hline No. & Acronym & Full name & Genus & NCBI & Identity & Similarity & Overlap length \\
\hline 1 & CLBV & Citrus leaf blotch virus & Citrivirus & NP_624333.1 & $48.8 \%$ & $71.9 \%$ & 2085 \\
\hline 2 & CLBV & Citrus leaf blotch virus & Citrivirus & AFA43536.1 & $48.2 \%$ & $71.9 \%$ & 2084 \\
\hline 3 & $\mathrm{AVCaV}$ & Apricot vein clearing associated virus & Prunevirus & YP_008997790.1 & $36.7 \%$ & $57.7 \%$ & 2104 \\
\hline 4 & $\mathrm{CPrV}$ & Caucasus prunus virus & Prunevirus & AKN08994.1 & $38.2 \%$ & $67.3 \%$ & 2093 \\
\hline 5 & ACLSV & Apple chlorotic leaf spot virus & Trichovirus & NP_040551.1 & $30.8 \%$ & $56.9 \%$ & 2107 \\
\hline 6 & GPGV & Grapevine Pinot gris virus & Trichovirus & YP_004732978.2 & $32.0 \%$ & $57.5 \%$ & 2089 \\
\hline 7 & CtChV-1 & Carrot $\mathrm{Ch}$ virus 1 & Chordovirus & YP_009103999.1 & $31.1 \%$ & $58.6 \%$ & 2093 \\
\hline 8 & $\mathrm{CtChV}-2$ & Carrot $\mathrm{Ch}$ virus 2 & Chordovirus & YP_009103996.1 & $30.4 \%$ & $57.7 \%$ & 2100 \\
\hline 9 & DiVA & Diuris virus A & Divavirus & YP_006905850.1 & $26.5 \%$ & $51.1 \%$ & 2084 \\
\hline 10 & ObRV1 & Ocimum basilicum RNA virus 1 & Divavirus & YP_009408144.1 & $27.2 \%$ & $51.3 \%$ & 2073 \\
\hline 11 & CVA & Cherry virus A & Capillovirus & NP_620106.1 & $32.4 \%$ & $62.3 \%$ & 1052 \\
\hline 12 & ASGV & Apple stem grooving virus & Capillovirus & NP_044335.1 & $31.9 \%$ & $60.9 \%$ & 1026 \\
\hline 13 & PVT & Potato virus $\mathrm{T}$ & Tepovirus & YP_002019748.1 & $34.5 \%$ & $64.0 \%$ & 989 \\
\hline 14 & PrVT & Prunus virus $\mathrm{T}$ & Tepovirus & YP_009051684.1 & $28.6 \%$ & $54.6 \%$ & 2084 \\
\hline 15 & GVA & Grapevine virus A & Vitivirus & NP_619662.1 & $27.0 \%$ & $52.5 \%$ & 2077 \\
\hline 16 & GCLV & Garlic common latent virus & Carlavirus & YP_004936159.1 & $29.4 \%$ & $56.0 \%$ & 2116 \\
\hline 17 & CVNV & Coleus vein necrosis virus & Carlavirus & YP_001430021.1 & $27.6 \%$ & $56.0 \%$ & 2106 \\
\hline 18 & PhlVB & Phlox virus B & Carlavirus & YP_001552317.1 & $28.6 \%$ & $55.8 \%$ & 2177 \\
\hline 19 & APV1 & Asian prunus virus 1 & Foveavirus & YP_009094347.1 & $28.8 \%$ & $58.2 \%$ & 2125 \\
\hline 20 & ASPV & Apple stem pitting virus & Foveavirus & NP_604464.1 & $28.8 \%$ & $56.7 \%$ & 2243 \\
\hline 21 & CTLaV & Cherry twisted leaf associated virus & Robigovirus & YP_009046478.1 & $29.5 \%$ & $58.4 \%$ & 2123 \\
\hline 22 & $\mathrm{CRMaV}$ & Cherry rusty mottle associated virus & Robigovirus & YP_007761581.1 & $29.6 \%$ & $57.6 \%$ & 2117 \\
\hline
\end{tabular}

https://github.com/lh3/bwa) (Li and Durbin, 2009). Sequence variants were identified using SAMtools/BCFtools programs (version 1.6; http://www.htslib.org) (Li et al., 2009).

Sequence comparison and phylogenetic analysis. NCBI BLAST searches were performed to identify and collect closely related viruses (https://blast.ncbi.nlm.nih.gov/Blast.cgi). Protein coding regions were predicted based on the outputs of BLASTX and ORFfinder (https://www.ncbi.nlm.nih.gov/orffinder). Functional domains were predicted by Pfam. Pair-wise identities among protein sequences were calculated using the FASTA program (version 36.3.6; https://fasta.bioch.virginia.edu/fasta_www2/fasta_down. $\mathrm{shtml}$ ). Multiple sequence alignments of sequences were generated using the MUSCLE program (version 3.8.425; https://www.drive5. $\mathrm{com} /$ muscle) (Edgar, 2004). Phylogenetic trees were inferred using the neighbor-joining method implemented in ClustalW2 software (version 2.1; http://www.clustal.org) (Larkin et al., 2007). We referred to the ViralZone web resource (https://viralzone.expasy. org) for the genome organizations of viruses (Hulo et al., 2011).

\section{Results and Discussion}

A BLASTX search was performed to compare the assembled contigs of the $S$. divinorum transcriptome dataset (SRR3716680) against the custom-built viral RNA-depend- ent RNA polymerase (RdRp) database. A 9214 nucleotide (nt) contig had sequence similarity with the RdRp motif sequence of the citrus leaf blotch virus (CLBV) (UniProt Acc. No. Q91QZ3). A BLASTX search of the contig against the NCBI non-redundant protein database confirmed that it is a viral genome closely related to CLBV, the type species of the genus Citrivirus of the family Betaflexiviridae (Vives et al., 2001). The contig was considered a novel Betaflexiviridae virus and named as Salvia divinorum RNA virus 1 (SdRV1).

A total of 26,102 reads from the RNA-seq data SRR3716680 were assembled for the SdRV1 genome contig. There were five single-nucleotide polymorphisms at nt positions 2748 , 4892, 6506, 6984, and 8054 (Supplementary Table S1), indicating that the SdRV1 genome contig is a composite sequence derived from a rather homogeneous viral population.

The SdRV1 genome was predicted to have four open reading frames (ORFs) (Table 1 and Fig. 1). ORF1 encodes a 2047 amino acid (aa) replicase polyprotein (REP). REP is composed of four functional domains predicted by Pfam: a viral methyltransferase (Pfam Acc. No. PF01660) at aa position 44-347, 2OG-Fe(II) oxygenase superfamily (PF13532) at 939-1047, viral (superfamily 1) RNA helicase (PF01443) at 1249-1500, and RdRp (PF00978) at 1622-2024. ORF2 encodes a 341 aa movement protein (MP), which has a viral movement protein domain (PF01107) at 14-196. ORF3 en- 


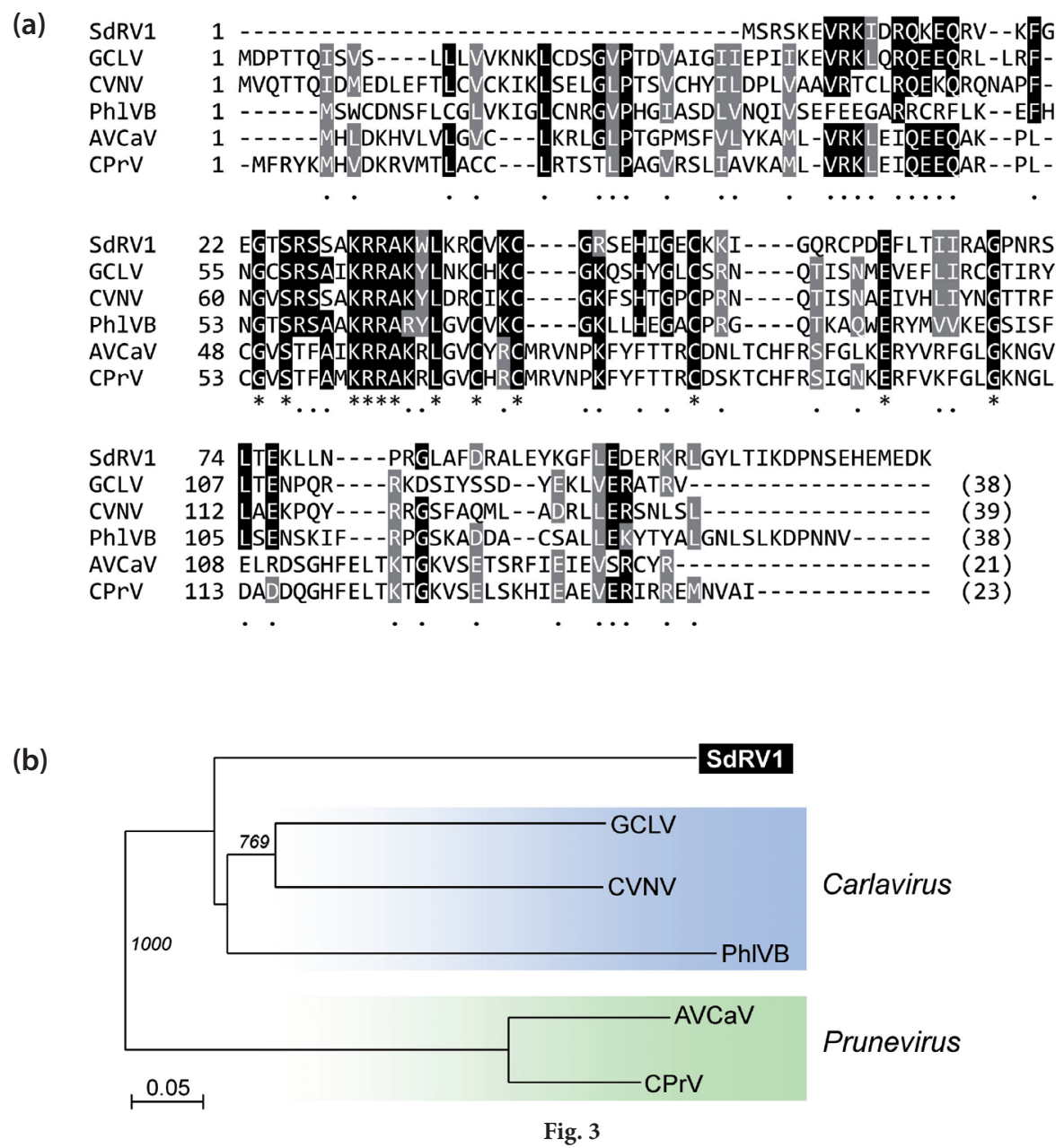

Multiple sequence alignment and phylogenetic tree of nucleic acid-binding protein (NBP) sequences (a) The NBP sequences of SdRV1, carlaviruses (GCLV, CVNV, and PhlVB), and pruneviruses (AVCaV and CPrV) were multiply aligned. (b) The phylogenetic tree inferred from multiple sequence alignment of NBP sequences is shown. The full names of the viruses are presented in Table 2. The NCBI Acc. Nos. of the NBP sequences are: SdRV1, AYE54587.1; GCLV, YP_004936164.1; CVNV, YP_001430026.1; PhlVB, YP_001552322.1; AVCaV, YP_008997793.1; and CPrV, AKN08997.1.

codes a 363 aa coat protein (CP), which contains a viral coat protein domain (PF00286) at 169-315. ORF4 encodes a 120 aa putative nucleic acid-binding protein (NBP) with a viral nucleic acid binding domain (PF05515) at 1-89.

NCBI BLAST searches were performed to identify and collect viruses related to SdRV1. A total of 23 viruses from 11 genera of the family Betaflexiviridae were selected including CLBV (Citrivirus), apricot vein clearing associated virus (AVCaV) (Prunevirus) (Elbeaino et al., 2014), apple chlorotic leaf spot virus (ACLSV) (Trichovirus), carrot Ch virus 1 (CtChV-1) (Chordovirus), Diuris virus A (DiVA) (Divavirus), cherry virus A (CVA) (Capillovirus), potato virus T (PVT) (Tepovirus), and grapevine virus A (GVA) (Vitivirus), garlic common latent virus (GCLV) (Carlavirus), apple stem pitting virus (ASPV) (Foveavirus), cherry twisted leaf associated virus (CTLaV) (Robigovirus).
A sequence comparison of REP sequences of SdRV1 and representative Betaflexiviridae viruses was conducted using the FASTA program (Table 2). The SdRV1 REP had the highest sequence similarity with the CLBV REP (NCBI Acc. No. NP_624333.1), which is a member of the genus Citrivirus, with $48.8 \%$ identity and 2085 aa overlap. The SdRV1 REP also had high sequence similarity with viruses of the genus Prunevirus: AVCaV with $36.7 \%$ identity and 2104 aa overlap; and Caucasus prunus virus (CPrV) (Marais et al., 2015) with $38.2 \%$ identity and 2093 aa overlap. Other members of the family Betaflexiviridae also had significant sequence similarity. The sequence comparison indicated that SdRV1 is closely related to the genus Citrivirus.

A multiple sequence alignment of full-length REP sequences of SdRV1 and representative Betaflexiviridae viruses was generated by MUSCLE (Supplementary Fig. S1). The 
SdRV1 REP had greatest sequence similarity with the REPs of citriviruses and pruneviruses. The SdRV1 MP and CP sequences also had greatest sequence similarity with the MPs and CPs of citriviruses (Supplementary Figs. S2 and S3).

We conducted a phylogenetic analysis using the multiply aligned REP sequences of SdRV1 and selected viruses of the family Betaflexiviridae using ClustalW2 (Fig. 2). SdRV1 formed a clade with members of the genus Citrivirus (CLBV), in agreement with the protein sequence comparisons. The next closest viruses were members of the genus Prunevirus (AVCaV and CPrV). The phylogenetic analysis implied that SdRV1 is a novel species that is most closely related to the genus Citrivirus in the family Betaflexiviridae.

However, citriviruses have only three ORFs encoding REP, MP, and CP (Hajeri et al., 2010; Elbeaino et al., 2014; Marais et al., 2015). In addition to REP, MP, and CP, the SdRV1 genome was predicted to have an additional fourth ORF encoding a 120 aa NBP. NBP is commonly found in viruses of the genera Prunevirus, Vitivirus, and Carlavirus (Adams et al., 2012; Rubino et al., 2012; Veerakone et al., 2018), but not in the genus Citrivirus. Among these viruses, only pruneviruses have the same genome organization as SdRV1, implying a possible affiliation between SdRV1 and pruneviruses (Fig. 2).

More interestingly, the SdRV1 NBP had greatest sequence similarity with the NBPs of carlaviruses, with 38-39 identical residues (Fig. 3a). The NBP sequences of SdRV1 and pruneviruses had the next greatest sequence similarity, with 21-23 identical residues. A phylogenetic analysis of NBP sequences also confirmed the closest relationship of SdRV1 and carlaviruses (Fig. 3b). It is worth noting that the SdRV1 NBP is most similar to the NBPs of carlaviruses, which have six ORFs with triple gene block (TGB) proteins instead of MP.

Phylogenetic analysis of SdRV1 proteins and comparison of genome organization revealed conflicting phylogenetic relationships. The sequences of SdRV1 REP, MP, and CP support the closest relationship with the genus Citrivirus. However, the genome organization of SdRV1 is the same as that found in the genus Prunevirus. Moreover, SdRV1 NBP had the highest sequence similarity with the NBPs of the genus Carlavirus. The uniqueness of the SdRV1 genome might be due to a complex evolutionary history involving the ancestors of the genera Citrivirus, Prunevirus, and Carlavirus. SdRV1 may be classified as a novel genus in the family Betaflexiviridae.

In conclusion, we identified a novel RNA virus named SdRV1 from S. divinorum. The genome organization and protein sequences revealed that SdRV1 has a unique phylogenetic position. SdRV1 could be considered the founding species of a novel genus in the family Betaflexiviridae. The genome sequence of SdRV1 identified in the present study might be useful for inferring the evolution of Betaflexiviridae viruses.
Acknowledgments. We thank Professor Philipp Zerbe (Department of Plant Biology, University of California-Davis) for information on the $S$. divinorum transcriptome data. This research was supported by the National Research Foundation of Korea funded by the Government of Korea (grant Nos. NRF-2017R1A1B4005866 and NRF-2018R1A5A1025077).

Supplementary information is available in the online version of the paper.

\section{References}

Adams MJ, Candresse T, Hammond J, Kreuze JF, Martelli GP, Namba S, Pearson MN, Ryu KH, Saldarelli P, Yoshikawa N (2012): Family - Betaflexiviridae. In AMQ King, MJ Adams, EB Carstens, and EJ Lefkowitz (Eds): Virus Taxonomy: classification and nomenclature of viruses Ninth report of the International Committee on Taxonomy of Viruses. Elsevier, San Diego, pp. 920-941. https://doi. org/10.1016/B978-0-12-384684-6.00078-1

Adams MJ, Lefkowitz EJ, King AMQ, Harrach B, Harrison RL, Knowles NJ, Kropinski AM, Krupovic M, Kuhn JH, Mushegian AR, Nibert M, Sabanadzovic S, Sanfaçon H, Siddell SG, Simmonds P, Varsani A, Zerbini FM, Gorbalenya AE, Davison AJ (2016): Ratification vote on taxonomic proposals to the International Committee on Taxonomy of Viruses (2016). Arch. Virol. 161, 2921-2949. https://doi.org/10.1007/s00705-016-2977-6

Anderson RI, Becker HC (2017): Role of the dynorphin/kappa opioid receptor system in the motivational effects of ethanol. Alcohol. Clin. Exp. Res. 41, 1402-1418. https:// doi.org/10.1111/acer.13406

Bankevich A, Nurk S, Antipov D, Gurevich AA, Dvorkin M, Kulikov AS, Lesin VM, Nikolenko SI, Pham S, Prjibelski AD, Pyshkin AV, Sirotkin AV, Vyahhi N, Tesler G, Alekseyev MA, Pevzner PA (2012): SPAdes: a new genome assembly algorithm and its applications to single-cell sequencing. J. Comput. Biol. 19, 455-477. https://doi.org/10.1089/ cmb.2012.0021

Butelman ER, Kreek MJ (2015): Salvinorin A, a kappa-opioid receptor agonist hallucinogen: pharmacology and potential template for novel pharmacotherapeutic agents in neuropsychiatric disorders. Front. Pharmacol. 6, 190. https://doi.org/10.3389/fphar.2015.00190

Chavan RR, Blouin AG, Cohen D, Pearson MN (2013): Characterization of the complete genome of a novel citrivirus infecting Actinidia chinensis. Arch. Virol. 158, 1679-1686. https://doi.org/10.1007/s00705-013-1654-2

Chavkin C, Sud S, Jin W, Stewart J, Zjawiony JK, Siebert DJ, Toth BA, Hufeisen SJ, Roth BL (2004): Salvinorin A, an active component of the hallucinogenic sage Salvia divinorum is a highly efficacious kappa-opioid receptor agonist: structural and functional considerations. J. Pharmacol. Exp. Ther. 308, 1197-1203. https://doi.org/10.1124/ jpet.103.059394 
Chen X, Berim A, Dayan FE, Gang DR (2017): A (-)-kolavenyl diphosphate synthase catalyzes the first step of salvinorin A biosynthesis in Salvia divinorum. J. Exp. Bot. 68, 1109-1122. https://doi.org/10.1093/jxb/erw493

Diaz-Lara A, Golino D, Al Rwahnih M (2018): Genomic characterization of grapevine virus J, a novel virus identified in grapevine. Arch. Virol. 163, 1965-1967. https://doi. org/10.1007/s00705-018-3793-y

Edgar R (2004): MUSCLE: multiple sequence alignment with high accuracy and high throughput. Nucleic Acids Res. 32, 1792-1797. https://doi.org/10.1093/nar/gkh340

Elbeaino T, Giampetruzzi A, De Stradis A, Digiaro M (2014): Deep-sequencing analysis of an apricot tree with vein clearing symptoms reveals the presence of a novel betaflexivirus. Virus Res. 181, 1-5. https://doi.org/10.1016/j. virusres.2013.12.030

Goh CJ, Park D, Lee JS, Sebastiani F, Hahn Y (2018): Identification of a novel plant amalgavirus (Amalgavirus, Amalgaviridae) genome sequence in Cistus incanus. Acta Virol. 62, 122-128. https://doi.org/10.4149/av $2018 \quad 201$

Hajeri S, Ramadugu C, Keremane M, Vidalakis G, Lee R (2010): Nucleotide sequence and genome organization of dweet mottle virus and its relationship to members of the family Betaflexiviridae. Arch. Virol. 155, 1523-1527. https://doi. org/10.1007/s00705-010-0758-1

Hulo C, De Castro E, Masson P, Bougueleret L, Bairoch A, Xenarios I, Le Mercier P (2011): ViralZone: a knowledge resource to understand virus diversity. Nucleic Acids Res. 39, D576-582. https://doi.org/10.1093/nar/gkq901

Jo Y, Song M-K, Choi H, Park J-S, Lee J-W, Lian S, Lee BC, Cho WK (2017): Genome sequence of grapevine virus T, a novel foveavirus infecting grapevine. Genome Announc. 5, e00995-00917. https://doi.org/10.1128/genome A.00995-17

Kim H, Park D, Hahn Y (2018): Identification of novel RNA viruses in alfalfa (Medicago sativa): an Alphapartitivirus, a Deltapartitivirus, and a Marafivirus. Gene 638, 7-12. https://doi.org/10.1016/j.gene.2017.09.069

Kivell BM, Ewald AW, Prisinzano TE (2014): Salvinorin A analogs and other kappa-opioid receptor compounds as treatments for cocaine abuse. Adv. Pharmacol. 69, 481-511. https://doi.org/10.1016/B978-0-12-420118-7.00012-3

Larkin MA, Blackshields G, Brown NP, Chenna R, McGettigan PA, McWilliam H, Valentin F, Wallace IM, Wilm A, Lopez R, Thompson JD, Gibson TJ, Higgins DG (2007): Clustal W and Clustal X version 2.0. Bioinformatics 23, 2947-2948. https://doi.org/10.1093/bioinformatics/btm404

Li H, Durbin R (2009): Fast and accurate short read alignment with Burrows-Wheeler transform. Bioinformatics 25, 17541760. https://doi.org/10.1093/bioinformatics/btp324

Li H, Handsaker B, Wysoker A, Fennell T, Ruan J, Homer N, Marth G, Abecasis G, Durbin R, 1000 Genome Project Data Processing Subgroup (2009): The Sequence Alignment/Map format and SAMtools. Bioinformatics 25, 2078-2079. https://doi.org/10.1093/bioinformatics/btp352
Li YY, Zhang RN, Xiang HY, Abouelnasr H, Li DW, Yu JL, McBeath JH, Han CG (2013): Discovery and characterization of a novel carlavirus infecting potatoes in China. PLOS ONE 8, e69255. https://doi.org/10.1371/journal.pone.0069255

Marais A, Faure C, Mustafayev E, Candresse T (2015): Characterization of new isolates of apricot vein clearing-associated virus and of a new Prunus-infecting virus: Evidence for recombination as a driving force in Betaflexiviridae evolution. PLOS ONE 10, e0129469. https://doi.org/10.1371/ journal.pone.0129469

Martelli GP, Minafra A, Saldarelli P (1997): Vitivirus, a new genus of plant viruses. Arch. Virol. 142, 1929-1932. https://doi. org/10.1007/s007050050088

Morozov SY, Solovyev AG (2003): Triple gene block: modular design of a multifunctional machine for plant virus movement. J. Gen. Virol. 84, 1351-1366. https://doi. org/10.1099/vir.0.18922-0

Pelot KA, Mitchell R, Kwon M, Hagelthorn DM, Wardman JF, Chiang A, Bohlmann J, Ro DK, Zerbe P (2017): Biosynthesis of the psychotropic plant diterpene salvinorin A: Discovery and characterization of the Salvia divinorum clerodienyl diphosphate synthase. Plant J. 89, 885-897. https://doi.org/10.1111/tpj.13427

Prosser SW, Xiao H, Li C, Nelson RS, Meng B (2015): Subcellular localization and membrane association of the replicase protein of grapevine rupestris stem pitting-associated virus, family Betaflexiviridae. J. Gen. Virol. 96, 921-932. https://doi.org/10.1099/jgv.0.000019

Riley AP, Groer CE, Young D, Ewald AW, Kivell BM, Prisinzano TE (2014): Synthesis and $\kappa$-opioid receptor activity of furan-substituted salvinorin A analogues. J. Med. Chem. 57, 10464-10475. https://doi.org/10.1021/jm501521d

Roth BL, Baner K, Westkaemper R, Siebert D, Rice KC, Steinberg S, Ernsberger P, Rothman RB (2002): Salvinorin A: A potent naturally occurring nonnitrogenous $\kappa$-opioid selective agonist. Proc. Natl. Acad. Sci. USA 99, 11934-11939. https://doi.org/10.1073/pnas.182234399

Rubino L, Russo M, De Stradis A, Martelli GP (2012): Tepovirus, a novel genus in the family Betaflexiviridae. Arch. Virol. 157, 1629-1633. https://doi.org/10.1007/s00705-012$\underline{1342-7}$

Valdes LJ 3rd (1994): Salvia divinorum and the unique diterpene hallucinogen, salvinorin (divinorin) A. J. Psychoactive Drugs 26, 277-283. https://doi.org/10.1080/02791072. 1994.10472441

Veerakone S, Liefting LW, Tang J, Ward LI (2018): The complete nucleotide sequence and genome organisation of a novel member of the family Betaflexiviridae from Actinidia chinensis. Arch. Virol. 163, 1367-1370. https://doi. org/10.1007/s00705-017-3701-X

Vives MC, Galipienso L, Navarro L, Moreno P, Guerri J (2001): The nucleotide sequence and genomic organization of Citrus leaf blotch virus: candidate type species for a new virus genus. Virology 287, 225-233. https://doi.org/10.1006/ viro.2001.1040 


\title{
SUPPLEMENTARY INFORMATION
}

\section{Identification of a novel member of the family Betaflexiviridae from the hallucinogenic plant Salvia divinorum}

\author{
C. J. GOH, Y. HAHN ${ }^{*}$
}

Department of Life Science, Chung-Ang University, 84 Heukseok-ro, Dongjak-gu, Seoul 06974, Republic of Korea

Received April 1, 2019; accepted April 4, 2019

Table S1. Sequence variations of SdRV1

\begin{tabular}{|c|c|c|c|c|c|c|c|}
\hline Position & Con $^{\mathbf{a}}$ & Alt $^{\mathbf{b}}$ & Quality $^{\mathbf{c}}$ & Con \# & Alt \# & Con \% & Alt $\%$ \\
\hline 2748 & A & G & 201 & 146 & 51 & 74.11 & 25.89 \\
\hline 4892 & G & A & 222 & 186 & 198 & 48.44 & 51.56 \\
\hline 6506 & C & T & 39.1421 & 215 & 48 & 81.75 & 18.25 \\
\hline 6984 & T & C & 222 & 148 & 123 & 54.61 & 45.39 \\
\hline 8054 & C & T & 130 & 188 & 55 & 77.37 & 22.63 \\
\hline
\end{tabular}

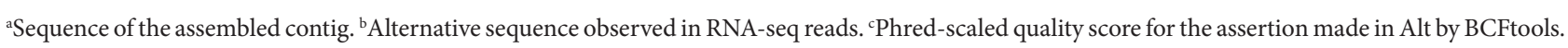


Fig. S1

Multiple sequence alignment of replicase polyprotein (REP) sequences of SdRV1 and representative members of Betaflexiviridae

SdRV1: AYE54584 CLBV:NP 624333.1 CLBV:AFA 43536.1 AVCaV:YP_008997790.1 CPrV:AKNō8994.1 ACLSV:NP 040551.1 GPGV:YP_-004732978.2 CtChV-1:YP_009103999.1 CtChV-2:YP 009103996.1 DiVA:YP $00 \overline{6} 905850.1$ ObRV1:YP_009408144.1 CVA:NP_620106.1 ASGV:NP 044335.1 PVT:YP_ō02019748.1 PrVT:YP 009051684.1 GVA:NP 619662.1 GVE:YP_002117775.1 GCLV:YP_004936159.1 CVNV:YP 001430021.1

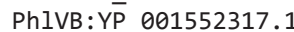
APV1:YP_ō09094347.1 ASPV:NP_604464.1 CTLaV:YP 009046478.1 CRMaV:YP_007761581.1

SdRV1:AYE54584 CLBV:NP_624333.1 CLBV:AFĀ43536.1 AVCaV:YP_008997790.1 CPrV:AKNō8994.1 ACLSV:NP_040551.1 GPGV:YP 004732978.2 CtChV-1:YP_009103999.1 CtChV-2:YP_009103996.1 DiVA:YP 006̄905850.1 ObRV1:YP 009408144.1 CVA:NP_620106.1 ASGV:NP 044335.1 PVT:YP $\overline{0} 02019748.1$

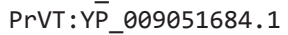
GVA:NP_-619662.1 GVE:YP 002117775.1

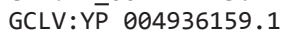
CVNV:YP_001430021.1 PhIVB:YP_001552317.1 APV1:YP 009094347.1 ASPV:NP_604464.1 CTLaV:YP_009046478.1 CRMaV:YP_007761581.1

SdRV1:AYE54584 CLBV:NP 624333.1 CLBV:AFĀ43536.1 AVCaV:YP_008997790.1 CPrV:AKN08994.1 ACLSV:NP_040551.1 GPGV:YP_ō04732978.2 CtChV-1:YP_009103999.1 CtChV-2:YP-009103996.1 DiVA:YP_006-605850.1 ObRV1:YP_009408144.1 CVA:NP 620106.1 ASGV:NP_044335.1 PVT:YP_0̄02019748.1 PrVT:Y $\bar{P}$ 009051684.1 GVA:NP 619662.1 GVE:YP_002117775.1 GCLV:YP 004936159.1 CVNV:YP 001430021.1 PhIVB:YP_001552317.1 APV1:YP_ō09094347.1 ASPV:NP 604464.1

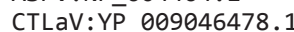
CRMaV:YP_007761581.1
- -MSLLVSKTPVECMLGNFEKSDIKRIYEPAVNYISSTA-IFRNKHFAYAMEPFLKKKLSS - - IGIELYPNGYIVHSHPFSKTLENHLLFDVLPGIIHDQ - -MALMSNKTAIESILGNFEKKHVDAIYNAAAOTILSHS-EFRNKHFAYSLNSYOKKIASK - -VGIELYPNGYLPHSHPLSKIFENHLLFDVLPGVVNTS - MALMSNKTAIESILGNFEKKHVDAVYNAAAQTIISHS-EFRNKHFAYALTSYQKKIASK - -VGIELYPNGYMPHSHPLSKIFENHILFDVLPDVVSTS - - MALLYRTPQVNLLGSFPQKHVEIIYNLQFERFKKICICRFCIFLTHSEKNQKKVASIRGWLGVPLHPTPYLAHSHPFSKMLENHILLNVLPGHITG- -MASVTVRTPMEKFFAANDKNDQRSILTSGVNFVKKFC-DDKGIHFAYYVNDRKKEALTN- - LGVTLHPIPFLTHSHPFCKTLENHLLINVLPNLLGNG - - -MAFSYRTPOEELLSRLPOSOOEVISGFOYERIOKEE -EKKVENFSFYLPEKTREWFTK - -SGVYLSPFAYVNHSHPGCKTLENHLLFNVVASYISK - - MTFFYRTPTEELISKFTSEEQARIYAPSQRYIENTEIGSNSLFFSYNLKEIQKKFFIS- -NGIELSPFSFKAHSHPACKTLENYFLFSFLPSFISH- - MSYGFRTPQEKILSTFSPSFIDNVQSTSGRTFEDEE-NRIGKFFNFNLDDRKKEFASN- - SGIYLSPYSYKSHSHPLCKTIENHLLYVVIPPLIQN - - MSYSYRTPOEKILSTLNPTLIDGVASFSGRIFEENE-SRLGKYFNYHLSDKKKEFAAO- - AGIYLSPYSFOSHSHPLCKTIENYLLYVVMPPMIAN- - MALSYRTPTEYLINQLPARLTDNIAVKQVDLIQSDE-DCYGSYLNYNLSKEQKKFLVD- -KGLYLSPYSWRHHSHPACKTIENWLLYKEIGSYAKHV - - MSLIYRTPVENLINQLPSRLTENVAVKQVDILQNME-ESIGKYFNFNLSKEQKKFLVD- -KGVYLSPFSWKHHSHPGCKTIENWLLYNEIGFHIRHI -MAFVAKFAEENYFNSLPSNVTDAFLRDGFNAEHNRF-EILSRHFAFALKPSORTYLND- - CGIOLAPIASKTHPHPVSKIIENHLLYCVVSNMISN- -MAFTYRNPLEIAINKLPSKOSDOLLSLTTDEIEKTL -EVTNRFFSFSITPEDQELLTK - - HGLTLAPIGFKSHSHPISKMIENHLLYICVPSLLSS - - -MSFSFRTPAELFVQSLPKEYAEACFKSHAANFQIRS-DKGVGLFDACSSVVKERLTK - -AGIPVSAFCNQEHSHPASKMIENHLLYNILPNYLNLK - -MAFSYRTPAEEFLSKLPSASQETVDEYALRSLRDQE-KASSRNYSYHLTDTQKSFCSK - -VGVPLSVNNFMVHPHPYCKTMENFLLHDNLFHYRNLI MSISVSSORVAVSNLYTNGSEESVKAIKELKSKRLLETE - TRLDGLFDYYIPDTLREILTG - -YGMEFSVHSFOGHAHPVSKMIENHMLYRVAPNYFSS MSLGASSQRVAYANLYANIGSDKLSEVRDRKASTVNSIE -AYASGLFDYYVSDDVYDFLAS - KGLPLSINCFRTHSHPISKMIENHFIFNLIGNNLAK - - MALTYRSPLEEVLTSFSASEQSLIAEPAITSYRGLE-RDLFHFFNYNVGPYAKEKLIG - AGVYLSPFSGVPHSHPVCKTLENHLLYRVLPSILDN - - MALTFRSPLEEVLTNFSSTEQSLISKTAINHYSNLE-SSLFNFFNLNVDAYSKEKLIN - SGIYLSPFSGVPHSHPVCKTLENHILYRVLPSLIDS - - -MALTYRSPLEENVAAYDASVQSTIASTSACYYKDTE-ADKFRFFNYFVNPTAKKKLIE - -AGLYLSPYSAMPHSHPVCKTLENYFLFEVIPSKIDN - - MALTYRSPIGEVLNRFTSEEQSRVSSTSVSRLTQFE -VNNHKLFSFAMSEKAKEKLIK - -VGIYLSPFSFEPHSHPVCKTLENHILYNILVNKLDN - -MALLSRTAAEEVIASFTSEEQSRISTQAVLALTNVE-KDKHDLFNYALPELAKMRLFN - SGIYLSPHSYRPHSHPVCKTLENNILFNILPSYLDN - - MALHTITPAENVLAQFSSEEASRIGASAISNFSKLE-ADYHNLFHFHLPAYAKKKLSE - RGFYLSPFSYETHSHPVSKTIESHLINIKLPNYINE - - MALHTITPAEGVLAQFSSEEASRIGASAISNFSKLE-SEYHSLFHFHLPAYAKSKLSN - -RGFYLSPFSYETHSHPVSKTIESHLINVKLPNYITE*.**.* :*.: :

A-- - FIFCSIKECKIRTFKA- - - - - - - - KSTSANISFLNRLIDGRDVARYPEVTH- - - - - - SSESQYLKGNSDSSPFSNSFKRT

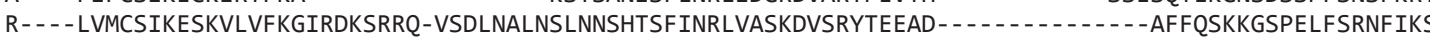

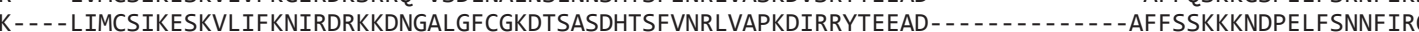

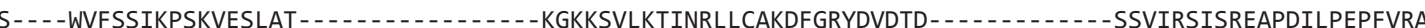

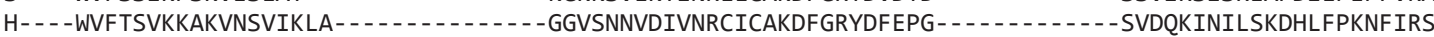

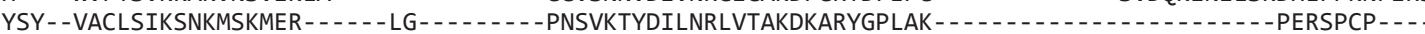
SGIRELFLFSIKKAKVTRLKN-

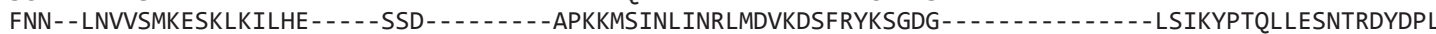
FNN - - LYVVSMKESKLRILHS- - - - - NS - - - - - - QLTGISLNLINRLVDVKDSFRYKDGGD - - - - - - YSSVKYPTDLLKWNSDKKYDPLLDC SKQQTIAFISLREGKLNAIKKIHFEKKNN- - - - - KVACEKICSFNRYYHTKDRLRYSDSSG -

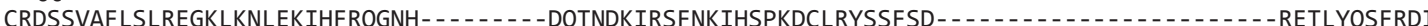

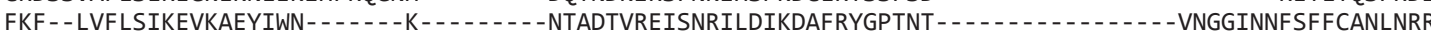

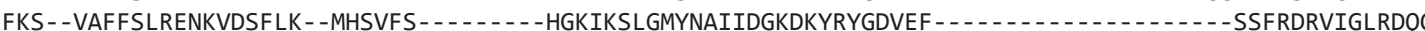
$\mathrm{N}-\mathrm{n}^{-}-\mathrm{Y}$ TAISIKDSKVRKLLK $-\ldots$

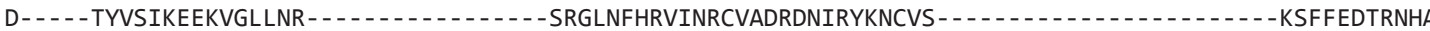

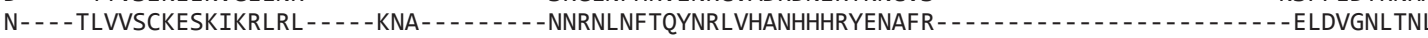
D- - - STFISFKEDKLVLLEN- - - - KK - - - - SRIDGNTCIINRLVHAKDALRYKDPLR S- - - - FLFVGIKESKLNFLRA- - - - - R- - - - - HQNLSMVELVNRYVTSADRTRYPNDFH - LSITAEQCFNRYDGFKHLGPGLRKLLPHCITV T- - - -FTFVGIKEAKLQFLRQ-- - - - R-- - - - -HSHLNLVQLINRYVTSADRLRYPSEFH- - ITPSKAIEVCEKWGRFGKSDSLRELLPACITH R- - - -FFFVGIKNEKLSMLKA- - - - - R- - - - - - NTHLSTVEKVNRYVTSADKVRYGSDFV - -VRPTEKIPGLIRORPCLDGVTLKDLVPELMRK S-- - FYAIGIKGSKVNFLKS- - - - - R- - - - - SKNLSMLEYMNRIVTSADKYRYGSDFT - - TLSTQQIDNLCSKQSLEKCGTLRELVPLVKMSK S--- - FYLVSIKKNKVDFLKR - - - - - R- - - - - HPDLQMVETINRYISSIDKTRYGGFFHVSPSKISAKFKCDRRTGFEDDASLIDLIPGCMEG D- - - FLIVGIKDNKLSVLRK- - - - - - EKKLRFLEAVNRCVTSHDIQRYGPSFH- - FEKAKSNWKTDFSEVNLSAGVQSLLPRVLFDK D--- FLIVGIKENKLSVLRK-- - - - - - DKKMRFLEALNRCVTSHDIQRYGPSFH-- -FEKARSNWRSDFSGVNLSAGVQSLLPRILFDK

VG- - - QGKNFFFHDEVHHWSQKDIFRFLKDFSP-KRLVFTVVYPAELLAGYANSQNPKMYKFK-IDG- - D- -KLFFFPDGVTSEGYQQPL - NLNWLFK LE - - - NKEAVFFHDEVHHWTKAOMFSFLKSTKV-KRFIFTVVYPPEILKKFANSONPKVYDFK -VDK - - - - - RLFFFPDGVKTEAYEOKL - NMEWLFS IS - - - NKEAVFFHDEVHHWTKAQMFSFLKRTKV-RRFIFTIVYPPELLKKFANSQNPKVYDFK -VDK - - - - - RLFFFPDGVKTEAYEQKL -NMEWLFS V-- - - KGRNVMIHDEVHHWTLDDMLGFLDRARP-NRFVFSVVYPVELLAGILESQNPKMYKFQDSKS - - - D- -KIVFFPDGRASEGYEQRA-NLRWLFC V- - - - RKKKIFIHDEVHHWSHLNMIOFLEETAT-PLLLCSVVFPPELLGGIKTPONSALYGFO-VDG - - D- -KLFFFPDGSRSEMYEOPS-NLNWLFE - - - - KKTNIFIHDEIHYWSRDQLETFLQVHRP-KNLWATLVFPPEILAGYKSSVLPFLYQFE-IHG - - K- -DLVYMPDGVRSESYTQPL-ENGFLLS K-- - - TGLDIFIHDEIHHWSKAQLISFLEVHRP-RNIMATVVFPIEILGGFKSSVLKFLYEFE-CRN-- - - - KLFYYPDGVMSEAYVQTL-ESSYLFK MDVRIEKGSNFLFHDELHYWTFSMMLDFLEKFEP-SHVICTAVFPVEILEGIKQSLYPEVYSFEILQT - - - - - NFVFAPDGVYSESYEQSV-NMKWLFS SIE - - KGRNFLFHDEMHYWSFDMMLDFLRKFEP-DNVLCTVIFPVEIFSGVKOSLFPDVYSFOIIKN- - -K - RFVFAPDGCYSESYEQSV-DMKWLFS GDQIG-PRASFYIHDECHYWSPNDLSNFLSRTKA-ESILATVIHPTEIDVGKDCSHLPFLYEFE - VSD- - - N- -NIFFFPDGNRSEGYEQPK - TAGWWLK GMKME -KRSCYFIHDECHYWNPADLDRFIRYTDA-ESIMFTVIHPVEVDVGKTSSHLPFLYEFC-IDG - - E - - TLHFFPDGNKSEGYEQPL-SAGWWLK FNNRAIKPDCFFIHDEVHFWSPAELCEFLFTVEP-KNVLATVVIPPELLEGLDYSFNSVAYDFKKVDG- - - - - - LYYFPDKSKGKPYOOPM-DP-WLLK LTRNK -FPKVLFLHDELHFLSPFDMAFLFETIPEIDRVVATTVFPIELLFGDKVSKEPRVYTYK - VHG - - - S- - SFSFYPDGVASECYEQNLANSKWPFT FIARVHHSTRIFLFDELHYWSMNSLSDFLDRSNV-KELLATIVFPIEILLGSKRSLNPELYEFE-ISR - - - G- - KLHFFPDGCTSESYSQP - - KDCDILK PN- - - OSKSWFFHDELHHWDVNEFADFLGNFKP-KKVIASCVFPVEVFSS-DESCNPSFYRYL - IHEKKKGEVRFTFFPDGSKESSYFOR - -SSDWIFR INKED-QSECIFIHDEVQYWSLDEMQRFLGSLSKVDRVVYSIIYPSEVEAGYSQSLFPEAYTFD-LKD- - - R- - RLVWYPDGKAEGAYTQPV-NP-WLLR MKGLA-EPDRVIIHDEVHYWNLKDFQRFLSYINC- -PLIYTVIYPAELHAGYPFSLYPELYDFR-VSE - - -DGKTFTWMPDGKCCGSYKQPV-NP-WLLS - - - - - KPRRLFLHDELHYWCERDLVTFLSAVKP-EKVLGTVVYPPELLKGVKFSLNKWCYDFD-VEG- - -D - -DLIFYPDGVRTESYTQPL - SGCFYLK - - - - - KPRALFLHDELHYWNLKELKVFLLAVKP-EKLLGTLVFPPELLQGVHESLNPWCYTFE-VDD- - - K - -WLHYYPDGVKTEGYTQPR-NSGYLLR - - - - SAKHLFMHDEIHYWSARDLITFLEVIQP-EVMYATMVYPPELLVGSKFSLFKWCYEFD-VIG - - - N- -DFFFYPDGVRTEGYLQPL-KNGYLLK - - - - KKRNFFLHDELHYWSSHDLMLFLDSINP-DHLLATIVFPPEILAGAQESLNPWCYSFQ-RHG - - - - - KLTFFPDGVQSESYTQPL-SAGYILQ - - - - ARKRFFFHDELHYWTKEALITFLDHVKP-EVMLASIVFPPEILAGAKESLNPWCYTFR - IVG - - K - -DLVFFPDGEQSEAYIQPV-AGSYLLR GRI - - QDAQIFLYDELHYWSMRDIVDFLEITRA-KTLIGSFVFPTEILAGSDRSLNPWAYDFK - IQG - - - D- -KLIYAPDGVWAESYEQPL-AAGQILK GKM- - - FDSQIFLYDELHYWSMKDIVDFLEISKA-KTIIGSFVFPSEILAGARTSLNPWAYEFK - IKG - - -D- -KLIYAPDGVWSESYEQPL-SAGQLLK : $* *$ * 
SdRV1: AYE54584 CLBV:NP_624333.1 CLBV:AFÄ43536.1 AVCaV:YP_008997790.1 CPrV:AKN08994.1 ACLSV:NP_040551.1 GPGV:YP_ō04732978.2 CtChV-1:YP 009103999.1 CtChV-2:YP_009103996.1 DiVA:YP_006̄905850.1 ObRV1:YP 009408144.1 CVA:NP_620106.1 ASGV:NP 044335.1 PVT:YP_ō02019748.1 PrVT:YP_009051684.1 GVA:NP 619662.1 GVE:YP_002117775.1 GCLV:YP 004936159.1 CVNV:YP 001430021.1

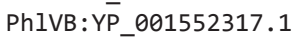
APV1:YP_0̄09094347.1 ASPV:NP 604464.1 CTLaV:YP_009046478.1 CRMaV:YP_007761581.1

SdRV1: AYE54584 CLBV:NP_624333.1 CLBV:AFA 43536.1 AVCaV:YP 008997790.1 CPrV:AKN0̈8994.1 ACLSV:NP 040551.1 GPGV:YP $\overline{0} 04732978.2$ CtChV-1:YP_009103999.1 CtChV-2:YP_009103996.1 DiVA:YP_006̄905850.1 ObRV1:YP_009408144.1 CVA:NP 620106.1 ASGV:NP_044335.1 PVT:YP $\overline{0} 02019748.1$ PrVT:YP_009051684.1 GVA:NP_б̄19662.1 GVE:YP 002117775.1 GCLV:YP_004936159.1 CVNV:YP 001430021.1 PhIVB:YP 001552317.1 APV1:YP_ō09094347.1 ASPV:NP 604464.1 CTLaV:YP 009046478.1 CRMaV:YP_007761581.1

\section{SdRV1: AYE54584} CLBV:NP 624333.1 CLBV:AFĀ43536.1 AVCaV:YP_008997790.1 CPrV:AKN08994.1 ACLSV:NP_040551.1 GPGV:YP_ō04732978.2 CtChV-1:YP_009103999.1 CtChV-2:YP_009103996.1 DiVA:YP $00 \overline{6} 905850.1$ ObRV1:YP_009408144.1 CVA:NP_620106.1 ASGV:NP 044335.1 PVT:YP_002019748.1 PrVT:YP_009051684.1 GVA:NP_619662.1 GVE:YP_002117775.1 GCLV:YP̄_004936159.1 CVNV:YP_001430021.1 PhIVB:YP_o01552317.1 APV1:YP 009094347.1 ASPV:NP_604464.1 CTLaV:YP_009046478.1 CRMaV:YP_007761581.1
A---SHLVDGDK-V---- -WTVVRHCSKFSHHLFEILPGE-LHTDSTFFFNDFDVVNMSIMFKNR-FRYYDL- -FPVNYQHLFKVYSYLNCLKNADVQSG A---SHLRSGDC -V-- - - WTVTRHKSIYAHHLFEISIGE-LVTDSKLFFSDYNSIDMSKIFLDR-FRSYEV--FPISIEHLYKVYSYLLCLKKPDLESG A---SHFKSGDQ-T-- - - WTVTRHKSIYSHHLFEVSMGE-LISDSKIFFSDYGSIDMSKIFLDR-FRSYEV--FPIAIEHLYKVYSYLLCLKKPDLESG A---SHFRTSGS-I - - - -WTVKRIYSAYSHHLFEVVPGN-YFTDEIRFFNDFETIDLQCIFKSR-FLCRDF- -VPISKDLVERVYSYLICLKKPDMQSA A-- -SYIHTSAG-T-- - - YTVKMVGSFYAHHLFOISKGE-KITDSVRFFADFNTIDMSVIHKER -FKYYDL - - IPIKKSHIEKIYTYLLCLKKPDVESA S---SSIIIKNRVTGVEIRYQVSLVYSLGSHHLFHIYPAEDLMKEEVRRFGPYDLFDVGSLFVKP-VRV-PI - QDFPLSVFKKIFIYMSSLKKPDVQSA T- - -NLIKTSKG-H- - - -YSVSLHRSVGSHHFFQISKYESEILQSHRAFGPYDVLDVGSLFRGK -VRV-SI - EGVSLTHFKKILIYLMSLKKPDVNSA A - - SSFKVRDQ - I - - - -YSVDLIKTIGAHHLFTIVKGR-RISRSIRLFKGFDTLDMSAFLGTK-YKM-PI - ADVHFSFFKKIVIYLKSLKKPDTQSA A---ASFVVDDL - L - - - -YSVDLIKTVGAHHLFAITKGK-RIVKEIRFFREFDILDLSAFSGTE-YKL-PV- -VDVHFSFFKKIVIYLKSLKKPDSQSA M- - -RRFYSDGE-V-- - - YSVTLLRTIGPFHLIYISRGS-LASESRRFFDDFNILDLPVKYAKNNLIKKMK - - LLLRNNFMIKIVSYIKSLKKPDKESA M- - -SRFISRGE-V--- - YNVTLLRSIGPFHLMFLSRGG-RVVESKRFFTDFNILDIPSRFNHQHFTRDVN- -MILRANFIKKIISYIKSLKKPDRESA CNKISMIKNGEIFS - - - - YSIGLLESVGANHLFSFQRNK - -VVESTRFFNDFDCLDMRKLLPIN-VENGKIKGYNIRTWVFKKILSYIVCLKKGDSESS C - - SGIQWANR - K - - - - IRVTKLQSLFAHHVFSFDRGR - -ACNEFNHFDKPSCLLAEEMRLLT - - KRFDK - -AVINRSTVSSLSTYMACLKTANAASA V- - -NRIVTKTGKI - - - - FSVELIHTIGANHMVMIKEGS-FDVDSERFFDRSSALTTSLLMPTR - - AGKA- - LRIRRKFLLRLIIYLFSLKKPDHHSA F - - -KYFEIGSE - I - - - - YTLSFLRSIKCHHLILIEKGK-LETNDYYISDEAECASLRMFGTAV-AGK - IT - -APIRVEVLKREMIYLLSLKKSDMNSA C - - -SKTEDSKGRS - - - - WTITKLOTIGAHHLFSAIKGS-YLTEESYKYDNFTIINPNDVLKGK - - RGGKP- - LYLRARMIKPTLLYLLALKKSDSNSA T- - -NKTMDLKGRT - - - - -WTLTKLESVGSHHLFLCTPGD-LVTEDEAVYNDFTLVDPKLFSSLT-QRM- - - - PKLRAGYMHKVIHYLMALRKPDAASA V- - -GTLKLLDGSV-- - - YHIDVVHSTFCHHLISITAGE-AVRAPTNSFSNFDATTCRGLPNIAFRSLGPC- - IAVPYPVISRVYRYLRTLQKPDLQSA L - - -SKIHLSDGST - - - - YCVDLVYSCYSHHIIALTLGD-AVRKPFNAFSGFDATTFQGLKKLDLRGIGPC - LPISFSVVNRIYRYLRTLQKPDLASA A- - -KRILLPDGGV - - - - YHVDVVQSKFAHHLIAITRGE-SMGPNVRAFGPFDATTCQGLEPLT-SDVSHC - FPVSFEVVSRVYRYLRTLKKPDVQSA A- - -SKIVCPSGQV - - - - YSVDLVYSAFAHHIVSITKGD-LPGLERRFFSNFEATGVKHLSGLS-YSVRDC - - IPVSSELISKLYRYIRTLKKPDLQSC T- - -GKITTPSGDI - - - - FQLDLLKSSFSHHLISITKGE-AIGQKMRFFNGFEAVAMKGLNPLR-RKVESC - - LPISKNTILKIYRYLRTLKKPDLQSA Y---NKIITQQG-V-- - - YSVQVRDSIYSHCLVIINRDN-LLNEEFRVYSEFDAVSIRRLNYLG-GNSDDI - - IPVRYEVILSVFKYIRTLKKPDLQSG F - - -NKIMTRNG - S- - - - YSVQVRDSIYSHCLVIINRDE - LLCEEFRVFSDFDAISIRKIGYLG-GNADDI - - IPVRHEVVLSIFKYIRTLKKPDLQSG

LAKLRQILGEDIEVKEFLFFEKFCTRVIEKGT - SYGLFGHSLFEKLS-CNFIKCLPNSVARLF - -PSWRKMN-TFDFLYNLDTLNIEVKRGKVFRDVLE LAKLRQIIGDDVEIKEFLFFEQFCKRLIERQT - SWGLFGHSFFEKLT-DMALSSLPNSIARIF - PQWKKKN - TFEFLFSLGTLVVDVERKVCFEHVLE LAKLRQIIGDDVEIKEFLFFEQFCKRLIERQT - -SWGLFGYSFFDKLT -DLALSKMPNVVARMF - PQWKKKN -TFEFLFSLGTLVVEIERRVCFEHILE MAKLKQLMGDDLDVRVQVFFRSLVHRILNESE - -CFSLFDVSIVNKWK - KKFLDFAPDWLLNGF - -MTWKSGN -FIKKLGEHEAAR-QVQEGAYPEGWMI IAKLRQLMEDEQDCRVVEFFCTFAKKLITDTK-GAINLFGDSFLQKAK-DSFIMALPNSIASCF- -DRWHGLN-IFHFLFTLDTIRVKVETKVVDRGYRT VAKLRQLSDADISIESVFMIQEFASRIEKNGV - - - ESWSCSFWGCMK - DWFFDKLP - -YREVL - -EKIGLADDFTRRLMKIKPLAFDIHTTDRPLTVRM VAKLRQLSNEDVDTNEMWVIRDLAERITH-- - - GVSKWSNVGIRTILRDWMVDLCP--FKKNF - -ERYHLIDDFDRYLMSIKPLSFSFNCSDDVDYAFE VAKLRQLVGDSISLTEVLFIEDFASRFQEHGA---AKLSNDGIFDQFI-HSLKSILPEFVRRLM- -GSFQRDN-LLKQISEAKAFEVRIETCSINRNYDN VAKLRQLVGDNISLTEMLFIEDFAERFQQLGS - - AKFSIDGIFDQMA-ESLKSLLPLFFRKML - -GSFGRDN-IMKOIAMAEPFSVSIETCRVDKNYDN IAKLRMLSEDEFSLEEMIFVDGLVDTLLKNGY - - KSIWENGWVEWFI-CGLKDCLPDALHSAMFRSHFKAKQ-NLDLLMNMKTLSIVVETEDFYPYSKV IAKLRMMSEDDFSIEEMLFVEGLIESMLKDGI - - -RSIWEKGWMDGFM-ATLRDLLPPSIHSALFRKDFKARE -NLMVLIDLKPLTITLPTADYHMR - - LAKLRQLSDSSPSSDELLLIGDFFDLMTRVKIFNKRSPWSFLSDAKNYVDSWIIOSP-FLRRIFPVGSRAITELIRDWIANAESLKIQTTCSSL - - - - VAKLRQLEKRDLYPDELNFVYSFGEHFKNFGM- - -RDDFDVSVLQWVK-DKFCQVMPHFIAASFFEPTEFHLN-MRKLLNDLATKGIEVPLSVIILDKVIAKIRQSSDDSIFCDEIMLADHVGKIFEKLDP - - ASPFGVKGVFDLLTSIFKDIF - - LLDGLFNWSDRRKSEKFVEFMRALDYQTNKVVTCTFSGGVMR AAKLRQLSQEDYHPQELCFFLNLAGRIESV-- - - KGIFKDRGLVSTVIDCFAQTFPDRIARWI - -SKTTDENSFLRLISSMEGMKLTIKRESFPVENFL VAKLRMLSSREENMDEALFVAQLAKQIKDTAL - - YDKMGNPNLRSILSESFYDIAGSLFTRLFNRPEYDARC - LEKFIRSCETTEIHVERR-YMEGIRR VSKLROLSKGDETTDEMIFSGTIATOIAELKY - -FTEVGGLIDLTRALKVGFARLFGOSI -EYFSNKKWYHLDCFTNTVOFMVKAEVRIKMGYR - - - - - MAKLSQIMPEPTGY -QIKFMTEFSKMVIDTPV - GASFFQVAIFEKFK - GFLTTFFPKYVAARF - DVARIRS - LDEFVEHMCPYTFKIKAVVHDMRLHQ MAKLSQILPEPTSF-QIKFIREFSELVIKTET - -CSNLFQNNVLLDIK -NFFASQLPKRLAATV - DAYKISS - LDEFLEKMEPYSFTIKLVEATALM - MAKLSQLIPEPSGV-EIKFLQDFASLIISTNT - - IKSILCPSRLPLFL-GGWLRKLPKPLAQRF - KTVAEVS - LDDFISIMEPFSFRQELCDVDWNYFH MAKLSQIVPNPTGT - EIKFTEDFARLTMDVQP - - MRSMLMPETAKLIGSLFSSFLPFKMARFF - DCYKACS-LDKFIHDLEPFSFSVETKVINSRSNP MAKLSQVCKDPNGY -EIKFFEEFSKLCLKCDT - - LNTNMIPDMKRIVQGFFLKLFPNPISRNF - KVVQQLH-LDNFIETLEEFNFSINTESLSLNWKD MAKHRQLVDEPTGF - EVRFIEDFVQFILENHE - KFNLIGQKFSNFLS-SACIEVLPRYMQRFF- -KSFKGYS-LGKFIEEIEPFSFTVKCRTYSRFSFK MAKHRQLVDNPTGF - EIRFIEDFVQFILEHHE - KFNLIEQKFSNFFS-SACINLLPRYMQRFF--NSFKGYS-LGKFIEEIEPFSFTLRCSTYSRFGFK

KC--- -PIEIIR - LNCEVFLDPLPFFSENENF - - - NDERVD- -DVVLETVILRGWNHQKRFNPENSCYRESAFDILSRTINSKDGISQGTSGSLKGGVV EW - - - GFEVVI - TDENAYLDPLSIFAINENF - - - NEDRVD- -DGYLERIRLPFWNLNDYDLKRKRVNAYNILSYRFEEERKIESAQKGPNK - - - - - EW- - - -GFEVVI - TDENAYLDPLSVFALNENF - - - -NEERVD- -DGYLDRVKLPFWNLKDYDPKRGRANKYDLLCYKFEEERKNDLRERGPHK - - - - - N-1- - LFLANY-Q- - - - MFDDLE -VKDDVMSTKLPEAIDTLFF - - -GGQLVK--GGDRRTVVIKSLDGLIKFSRSKNLYKMHIIFLLNPSLIRGN- . - . - . - . - . II - - - -DQIWEERLSSFDNISNIVFYGRREWLNNGVLPKVKK - -KGLAKLIPGREVDSHNYPREIYSDLLSSTSIWRSYDDDFRHRSA- - - - - - - - PEEDLPDFIDDFWFGSEKKMIRDPLVKMIEMGRESQGYSVVP - -TSYQQAAVCIFSMDENCIGWRNRGVKVLLRASRSSYT - .

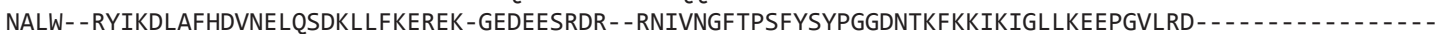

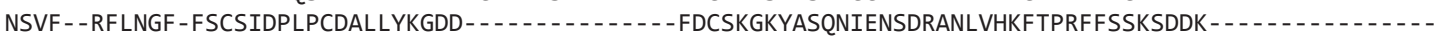
DCI - - KEIKEYFLNSCDYLQRDNIDK- - - SIIHRSVGIDIQYLN- - TFSDSFEMIKTDSISEFGEHILGNIS- - NFIETR-FHARMFDIAQAIGVNLDLL S----GFLAEF-FLDNDCEASEGL - - EFDVKDGISEQFILDLMEEKMSGGTDAKMDT- - - - - - - E- -GASFKV-QNVMDWVEDDSANALSEVN-

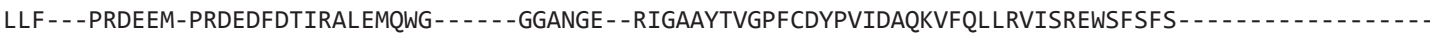
- - - DIIFNFDRDIEGFEHEVDIVAELEAFANPKNPHAHN- -AKTTPYFCEPYCDAVEFCVQKMAKLVLVK -

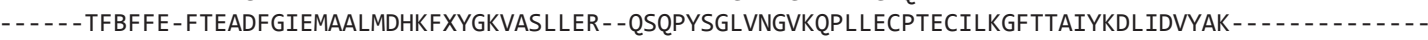
- - - - MFEAVTLGETMSSEEVLKILAKLDMGAPTSLEGLTGKFKYSSEAYHGLHHVLDGCGPHVFRRLVRKIADSFCNEFGIYRKSSYVSSVAITVLQR DL - - - EFVNLT - FGDTDFNVEDSFAEAWGTK - - - - KDVVN - - ITTVHHSPYLVSKFESYDHQFHSILSVKSISALTRIAKIVLSLYDPCV- - - - - N- - - - SFIEEEEARERDGDPIYKQFSKSQSF - - - - - NST - -AYPDCLFHASQSVFSNPHPNLIRRLVTLFISTWVGKTEADYYTSLLSL - - - - - - T- - - - SFIEEE - EAAVADKDPLCLTLKLANN - - - KPSCFE - -SYPDLIFNAHTLVFASSHPSITLMLVKSFINMWVGKTNDRYYOSLVAL - - - - - - - - 
SdRV1: AYE54584 CLBV:NP_624333.1

CLBV: AFA43536.1 AVCaV:YP_008997790.1 CPrV:AKN08994.1 ACLSV:NP_040551.1 GPGV:YP_o 04732978.2 CtChV-1:YP 009103999.1 CtChV-2:YP_009103996.1 DiVA:YP_006905850.1 ObRV1:YP_009408144.1 CVA:NP_620106.1 ASGV:NP 044335.1 PVT:YP_002019748.1 PrVT:YP_009051684.1 GVA:NP 619662.1 GVE:YP_002117775.1 GCLV:YP 004936159.1 CVNV:YP_001430021.1 PhIVB:YP_001552317.1 APV1:YP_o09094347.1 ASPV:NP_604464.1 CTLaV:YP_009046478.1 CRMaV:YP_007761581.1

SdRV1:AYE54584 CLBV:NP_624333.1 CLBV:AFĀ43536.1 AVCaV:YP_008997790.1 CPrV:AKNO8994.1 ACLSV:NP 040551.1 GPGV:YP 004732978.2 CtChV-1:YP_009103999.1 CtChV-2:YP 009103996.1 DiVA:YP_006905850.1 ObRV1:YP_009408144.1 CVA:NP 620106.1 ASGV:NP_044335.1 PVT:YP_002019748.1 PrVT:YP 009051684.1 GVA:NP_619662.1 GVE:YP 002117775.1 GCLV:YP_004936159.1 CVNV:YP_001430021.1 PhIVB:YP 001552317.1 APV1:YP_o09094347.1 ASPV:NP_604464.1 CTLaV:YP 009046478.1 CRMaV:YP_007761581.1

\section{SdRV1: AYE54584} CLBV:NP_624333.1 CLBV:AFA43536.1 AVCaV:YP_008997790.1 CPrV:AKN08994.1 ACLSV:NP_040551.1 GPGV:YP_004732978.2 CtChV-1:YP 009103999.1 CtChV-2:YP_009103996.1 DiVA:YP 006905850.1 ObRV1:Y $\bar{P}$ 009408144.1 CVA:NP_620106.1 ASGV:NP 044335.1 PVT:YP_-002019748.1 PrVT:YP_009051684.1 GVA:NP 619662.1 GVE:YP_002117775.1 GCLV:YP 004936159.1 CVNV:YP_001430021.1 PhIVB:YP_001552317.1 APV1:YP 009094347.1 ASPV:NP_604464.1 CTLaV:YP 009046478.1 CRMaV:YP_007761581.1

PTIKRICW-VSGGPFLTGLDSILGYEVQLSNKRKNFLFDFVCHILREEKIHIDHQDSKFLENMNVCLSIEKLGIIEAVIKMGERAAIINRSMIDFGAKLL - -MLQIEW-YG - - - - - - IKEF - - - - - -KVDPFISNSITEFTLLEALLGKRIDPKKYSYSKQACTLSNYLTFLCAEGLDGFNLEEHLERRL - - - - - - - MLQIEW-YG - - - - - - - IREF - - - - - - -DDPFIANGISEFTILEALIGKRIHKERYSYSKQADVLAKCLSFVCEIGGGGEGLEFVLERRL - - - - - -

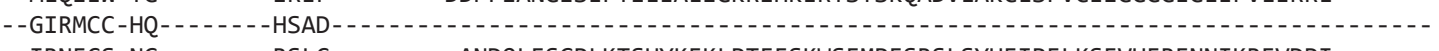
- - IRNFCS-NG - - - - - - RSLC - - - - - - ANDQLESGPLKTSHYKFKLPTFFSKWSEMPFSRSLSYHEIPFLKSFVHFRENNIKRFVDPI - . - . - -SPLVIL-RS - - - NRAY - - - - - -

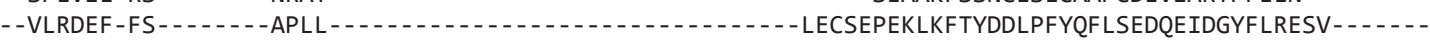

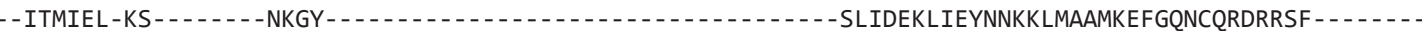
- $M S K S D F-Y R-\ldots$ --IIRSSF-RG- - - - - EYIY- -DEQMEF-KG- - - - - - VQED- - - - - -

-GKRFDY- - - DEVISR-FD- -

- EQRIEI-PG- -EIQELSFEEARFYHGIPGMKRAFD- - - - - - - - FLDISW-ND- - _ -EKRREY-QG--.-- - - LRGV-MVQYCE-RG -

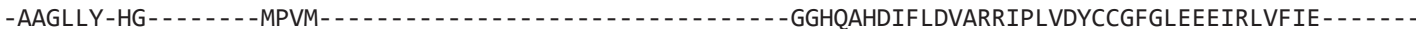
-DVISCW-VA- - - - - VDVE- KTNSNLSFIFG - $\ldots$ TDLN - -VEAFSE-SR - - - - VTNL - -KKSLSQ-KG-RQALNQ-KG-

IKERNDLRLMSKSNYDFLIESGGLSKTCFDCFFEAQLEADPFLEEYADCFSSNPCSHFFNCDIDVDG-NLKEVTFCRDGEFVQLIWENSLKTRGTVPDFP - -KAAGHDVSDDEEEELTSAEQA - . - .

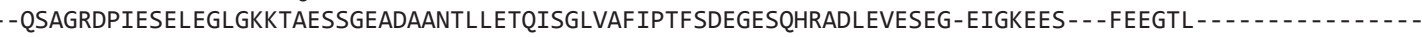
- FDMIIDEMNQLDLDIMNDGEEAAVEILDVNSNLDGKQSEHHDEEFV-HRREKKELSLKCLDFHI - _ -LDSIKDKAFHYSEGFF - _ -

-
- RKLSLAADIK- _. -

-RAHELI - - EAIADG-GVPERK - - - RQPYSM- - - - - - - - -

LATYNKYVHMRHSDWKRR

-KFIGLVRKHSRS-

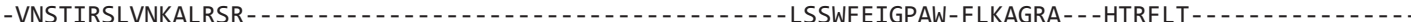
KFLRSPIWPNDLGLVEFR- -

- -KRAQGKMRKRFHFELGIR-KRLRLRNS-

TLNRTPSNDVCTSSSSDVSSCFGENVDDDCQNVDYSKMFCRVECDSLLGDIIDVPADGDCFLWSYKKAMNIES - TCDEIRSAFSEWL - -AH- -SKNLGVL - - - - - - EPSLEERGQFSTDYHSEKFEINYNDIFNPHNCMNTHGDEIPTPSDGNCFFSAFTETFEVE - -RPDTLRSDFSDWLMEFN- - GGSYASL

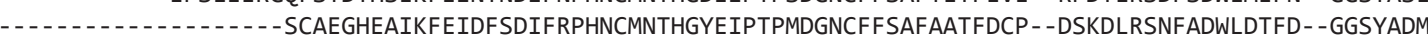
- - VSFPGDSCSVLMGNEPIAVADE- - (1) - LNTLDKVIAENGEKSD- -VPILSAD SCVHETLDDMLDVLSEIKMAYPLTTDV P P P

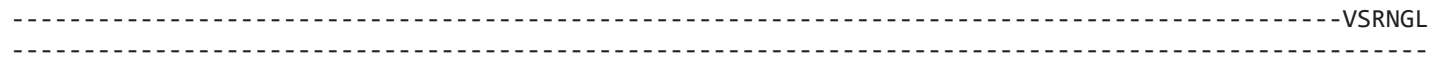
-SAHALRVNIKDRT--.-- - PNPYGPM -VLLTNYAHWGLPKR--EGIREVV CAYPDQAKSYKALDEAY--STVVADL CTPEAIVATKLMRGMWDNVVNELAAS SYPHDAIVYKEAKQGWS - -HVLKELA QTAPTNSKSNTSSSEGASNHQREORDOCDEATLOVN--FQREIEF -DQFIKGCQRDNSLHNGR--MSLRQVL SSVVSELLSISSECPPSSTLTKTSGLOOEL - -WKMKKEV CYKSLLADFKKMSEECS--PILTLIA

813 
SdRV1: AYE54584 CLBV:NP_624333.1 CLBV:AFA43536.1 AVCaV:YP_008997790.1 CPrV:AKN08994.1 ACLSV:NP_040551.1 GPGV:YP_ō04732978.2 CtChV-1:YP_009103999.1 CtChV-2:YP_009103996.1 DiVA:YP_006905850.1 ObRV1:YP 009408144.1 CVA:NP_620106.1 ASGV:NP 044335.1 PVT:YP_002019748.1 PrVT:YP_009051684.1 GVA:NP 619662.1 GVE:YP_002117775.1

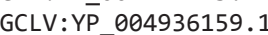
CVNV:YP 001430021.1 PhIVB:YP_001552317.1 APV1:YP_0̄09094347.1 ASPV:NP 604464.1 CTLaV:YP_009046478.1 CRMaV:YP_007761581.1

SdRV1: AYE 54584 CLBV:NP_624333.1 CLBV:AFA43536.1 AVCaV:YP 008997790.1 CPrV:AKNÖ8994.1 ACLSV:NP 040551.1 GPGV:YP_004732978.2 CtChV-1:YP_009103999.1 CtChV-2:YP_009103996.1 DiVA:YP_006̄ 905850.1 ObRV1:YP_009408144.1 CVA:NP 620106.1 ASGV:NP_044335.1 PVT:YP_ō02019748.1 PrVT:YP 009051684.1 GVA:NP_6̄19662.1 GVE:YP 002117775.1 GCLV:YP_004936159.1 CVNV:YP 001430021.1 PhIVB:YP 001552317.1 APV1:YP_009094347.1 ASPV:NP ${ }^{-} 604464.1$ CTLaV:YP 009046478.1 CRMaV:YP_007761581.1

\section{SdRV1: AYE54584} CLBV:NP 624333.1 CLBV:AFĀ43536.1 AVCaV:YP_008997790.1 CPrV:AKN08994.1 ACLSV:NP_040551.1 GPGV:YP_004732978.2 CtChV-1:YP_009103999.1 CtChV-2:YP_009103996.1 DiVA:YP_006905850.1 ObRV1:YP_009408144.1 CVA:NP_620106.1 ASGV:NP 044335.1 PVT:YP_002019748.1 PrVT:YP_009051684.1 GVA:NP_619662.1 GVE:YP_002117775.1 GCLV:Ȳ̄_004936159.1 CVNV:YP_001430021.1 PhIVB:YP_001552317.1 APV1:YP 009094347.1 ASPV:NP_604464.1 CTLaV:YP_009046478.1 CRMaV:YP_007761581.1
ADSVSVSNTFFEHEHVYFFAE - - - -FAQVTICVHSVEATFLFGDSGCKIHLNCDGTHFSPIILYNEKKKTFDQ- AEMIRPNGVFMEAELIYLFCVFRGVTLIIHDRTHEKENVYAVHRGFEEGHMVHRGNHFVGIETYNISTLTSDP GVKIRPNGVFMEAELIYLFCIYREVTLIMHDRTNDRESVFAIHLGFEEGHMVQRGDHFLGIETYRIDGFASDPSSGVSKEHTGLLGKTTYPSGP _. EAQILREGEQFDEWMIMFTVN- - - -VMNLSLKIFQGDVSIPRTLSPKLVNTHGNDAKEIAILH-RGNHFLGLLKKCTSESGAHDFDGNQPVEASSDNHFD

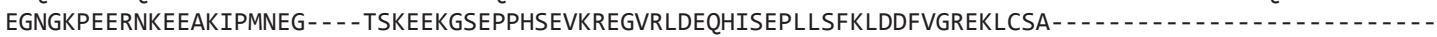
ESSESVNVKLLNESLTYDAEP - - -SECEVPSVESKHEPEQIESSGEIIPESWTFVDNNNKEFYCDGAFSLEL IQLEKDVETKLEEISANANEN- - - EDSVTMVSVSSSSSSSTSGREIEIDTSDLSDHLNDIRGIKPDDRWSDL IISPMPQIVTVEEIKDCLSVA- - - EVESSIDPQEGNFQEKAEGSEENLFFSNSDDSEMLDNIKRVKIEDENL - - - - - - LSYGYSHRIDSLCE - - FGNNHMEKMRIENRFWFLNDESSDYFDTMDLVEGINPLVNRS- - FDFIKNCYPDSFDIIRNNKEFQ VDSMRQNTSFSCEVFVDLFPS- $-1, \ldots$ - EAESEEYFSENGYIFMPSK- $-\ldots$ KARTPSVVLHREYITKVEFSSMWDAEEEISQKEGGLKVMEGV-IHPNTLSSDCAEDSESCGSVSGNDESMSSEEKEECEYINGRFSPNG QAGLEEGVITEEEAELRLLPT - - - TKPKITEIHMDDDTPGTSGE- - - - SDVEKFKSVRSLCREEIYSEKL CDAGDPVGELNEFKRMLGHPN- $\ldots$ LGSLARNKLIISAAAPKFDKT - - -SKVPREGVAEEIPAHCARTSAAFASAPCGSTASSECCVFELNPPAQIA SALIKRIGITLSDRNTPVAPL - - - - - EVVLPPAPEPIKQEGSNGSVGVSNELAEDAIANFTPPAFIRNF GGSRSLKHREMDYWFEKDRSS- - - KDSGGTVENDEDDKGEPEGVVSSSSDDGSDDKSSQEYIEVDTKVVRRMCMGSLPEANSFKSFTPCVEAVAETSYEVQPLEPEPEANGLVLGCIPDASTPAFTFNSANQKDRLFSSVVEENI KGPKLQALFDVSELSIIHNVE - - - MENAPEAGSTLDAGIKPTSSPLEVVPIENARCNLAPCKCDLNCFIQPA VTKQAPVLNEEQKPYKIPSER - - - EKCSTQAIEKNEEPKSEVVSTKGETPPETSKTTSKFGELLATPEATAV- - - - - - SEPTDNVLASSDLFIS TGGPFEQLNAMKKDFVLINQPSVGKFEKVKKCISKSTDASPDGGKEHKLEPQHSEGVNAKTDEVIPKRVDQPE- - - - - PKTEEGKTGSSDQFIS

SEIDGCFKVMNDGRLSQFNFDSSDSLNFKW--RGRESAFFSKVDA-DYGHNGMIYPHNGWVDDFDKIVQICD- -PSG- -NYNSVLVQW-YKKGAGIGLHR LLGDIPCGFSEEITKFHFRPDHFNCAQF - - - RGRKAAFITKVDA-DYGHNGMVYPHNSWVPSLEEIIQICG- - QGD - -DFNCALINF - YEANSSLGFHR NLSELPCGYSEELRNFHFKPEHFNCAQF - - - RGRKGAFLTKVDA-DYGHNGMVYPHNAWVPSLDEIIRICD--HGD- -DFNCALINF-YGPNSSLGFHR - IINALQGLKPKSF - - - - RGRSSFFFARSNEIDYGHNGFKYRTENWFAELDDFIPSDL - - - - - - IFNACLVQV-YDKGSKIGFHK ETFETGFNDFEELRKVVGSISMFKKTPL-- - KNRDAFFFSESKSIDYGHNRIKYAHNHW-NGVDQLLPSSL - - RN - -DYNAMLIQV-YKEGGSIGMHR - - - - - GLIKTVGNDYLLLARQIECMPLSQL - RGKKAAYFCLDFPMVYFHDKVSYPTFEATGEIRHVMMKARSKWGI - -DFNSALIQV-YNDGCRLPLHS - - - - - - - DFASILEGLKPLNL - - -RGRKAFFFHSNGL-PYFHDSVLYRTLEVPGWLEEIFFKANEHFDV--NFNSCLMQV-YEQDGSVGWHF VVEIKDSSFLQCGIDLDNLLKGIKSQKL - - - KTRKAFYFCKDANFDYGHDKVKYQNMGWPNFISELNKIACDVTGF - -KFNSVLINE - YTRGGRIHWHA - - - - - - - - - - - NLQERKIEGMKGKKI - - - -SNPERNWILNSGSLKIDYSRLVRARR - -FRLRRDFLDPIS-- - - - -KGKSPRKQLFLESTGNIKSNP KAKKVNVLKERLTGEVSFEEARLORMRYSDS - . - . - . - . - - OFVAGLAPK-LRYVKMLTWKK SFVESLSMLLNSKAAYLHGGRKTILFSTVTGLDYGFGPHKYKQIP-CDFMEDLLKKVG- - - - - FNSCLAQM-YGEGGSIGAHY -KGREVAFYSRHSK-EYKYNGGSHRSLGWDEALNELTQELG - LDD--SYDHCLIQR-YTAGGSIGFHA

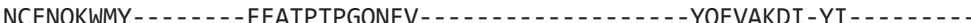

DLSCSCDRTLAVLNVLAYEALELEFPDQLPGCRAGWYVKGPQVADVGGFLGCQQLRWLDFFDSFLELHG-AQPG--SYNSCFAQV-FDLDSTFEYHS - - - SCSCGLEMPISSVVGGDFVYFDLPDVLPGRRAAWFTKDGSTAYTYKGGKHASMGWDERLDLLLEIHG-FEGS--LFDSALVQE-YEQGARIGFHS - - - - TCSCGVSMEIKTLLGSDMHAFKAKDRLKGRVGGWYSKDGT - PYLYTGGSHISQGWPDWLELWMQINK - -VPD- -KYNSCLYQI -YEEGASVGYHA - - - - - TDIGILSPMELVLPSSAEATPL - - - NGRQCYFFTRCGCIDYGHNKIRYKPNKWFSGLDSILKDEST- - - - - YYNACPLQI -YEAGAGIGFHS -DVNSLHGNLVFLDFIGGSKGRGASFYSRDLK-GYSYTGFSHVSRGWPAFLDKFLSDNK - IPLN - -FYNOCLVOE-YSTGHGLSMHK SIIKTGPFGDHGVIEFIRSLSFNDGHSH - - - NGRKALFFSRGGF-AYGFNSVTYQSSGWPSAFEEI - - - - - - YGD- - RFNSCLVQK - YEKSAKLGLHK SIIKVGPFKEPSTISFVEGLDFSKGHNH - - - KGRKSLFFSEGGF-SYGFGSIVYPSQGWPNAFKEL - - - - - - YGD - - RFNSCLVQK - YNADATLGFHA

DNESVYG - -DDPILTVNLSGKCTFSVEVEGNLK- - - - - - SFELSDFDYFVMPHNFQK - - RARHSVHAI -DARVSLTFRLHKR - - - - - - - DDEDCYD--DDPILTMNFNGTALFEIKNVV- - - - - - SSKLDHKDFILMKSGLQK - - REKHRVQYTSEGRISLTLRVQKR - - - - - - - DDENVYDLDRNPVLTVNMIGEGMFSVKMGKYEH- - - - - - - - SFPMSPGDMILMKNGAQK - - - RMKHSVIAK-DRRVSLTFREQIR - - - - - - - - - DDEHVYDSSDNPVLTINARGRGIFKYKVNKTGAVT - - - - - ECLLDEGEAILMKEGAQL - - RGKHSVHAISQ-RISITLRDQKR - - - - - - - - NIKHV - - - -DDPFSTMEKHKRDRLNKV - - - - - - - - IKYYIGGVEYEMPSSQVS - - ELEEITEITPINRLSSSNPMDEK - - - - - - - -

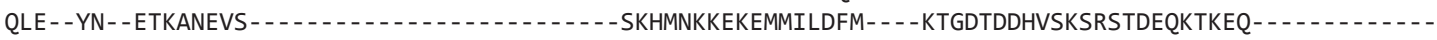

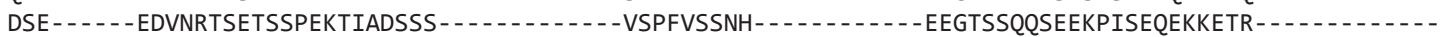

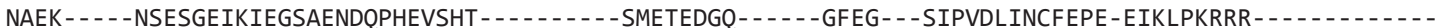

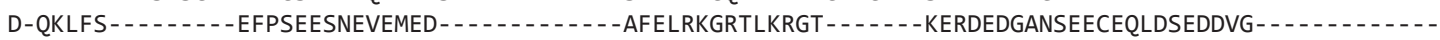
DDEKVYD--DDEILTWNLEGEADFTMFKKTGTE- - - - - - - SVHLTRNQVLVMPKGCSRGEEKFKHAVENCTEGRISITFRHQKR - - - - - - - DDEPCYL - PGGSVVTVNLHGDATFEVKENQSGKIE - - - - - - KKELHDGDVYVMGPGMQQ - - - THKHRVTSHTDGRCSITLRNKTV - - - - - - - - - - - KVYLITNPPLHELLLEGLITLE - .

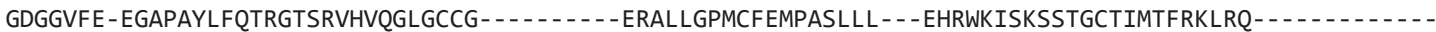
DDESIFK-VGSEILTMQLKGTSRFAIQGSRCYG - - - - - - -SSALLGGCHFTMPAGFQE - - - THKHSVAECSSGRTSITFRVLKG - - - - - - - - DDEPIFK-KGEE IHTCNLSGKAVFEIKCRNGAG - - - - - - GEYLEGPMQFTMPMDFQG - - THKHSVSGTTKGRESVTFRVLRA - . - . - . - . DNEKVYH--RSPIKTINFCGEADFVVKAKGKRDVGVNA - - - - TCHMKTGQFFTMDSNFQS - - YYYOHSVONCSEGRVSLTFRYHVN - . - . - . - . DDESIYD-INHQVLTVNYSGDAIFCIECLGSGF - - - - - - EIPLSGPQMLLMPFGFQK - - EHRHGIKSPSKGRISLTFRLTKEGDSQVPIQEVVTI DDEDCYD-DEHEVMTVNLFGTATLIFTADGAKGLERADPSKFLEITLSHGEYLLMPNGFQK - - -KFKHGVSCTSAGRISLTLRKQAR - - - - - - - - - . DDEQCYD-QDHEVLTINLFGSATICFTKGDFSALNTSNPKLYLEVGLDHCDWLLMPRGFQR - - NYKHSIKGTSEGRISLTFRKQRR - . - . - . - . - -
990 
SdRV1:AYE54584

CLBV:NP_624333.1

CLBV:AFA43536.1

AVCaV:YP_008997790.1

CPrV:AKN08994.1

ACLSV:NP_040551.1

GPGV:YP_- 004732978.2

CtChV-1:YP 009103999.1

CtChV-2:YP_009103996.1

DiVA:YP_006-̄905850.1

ObRV1:YP 009408144.1

CVA:NP_620106.1

ASGV:NP 044335.1

PVT:YP_002019748.1

PrVT:YP_009051684.1

GVA:NP 619662.1

GVE:YP_002117775.1

GCLV:YP 004936159.1

CVNV:YP 001430021.1

PhIVB:YP_001552317.1

APV1:YP_009094347.1

ASPV:NP_604464.1

CTLaV:YP_009046478.1

CRMaV:YP_007761581.1

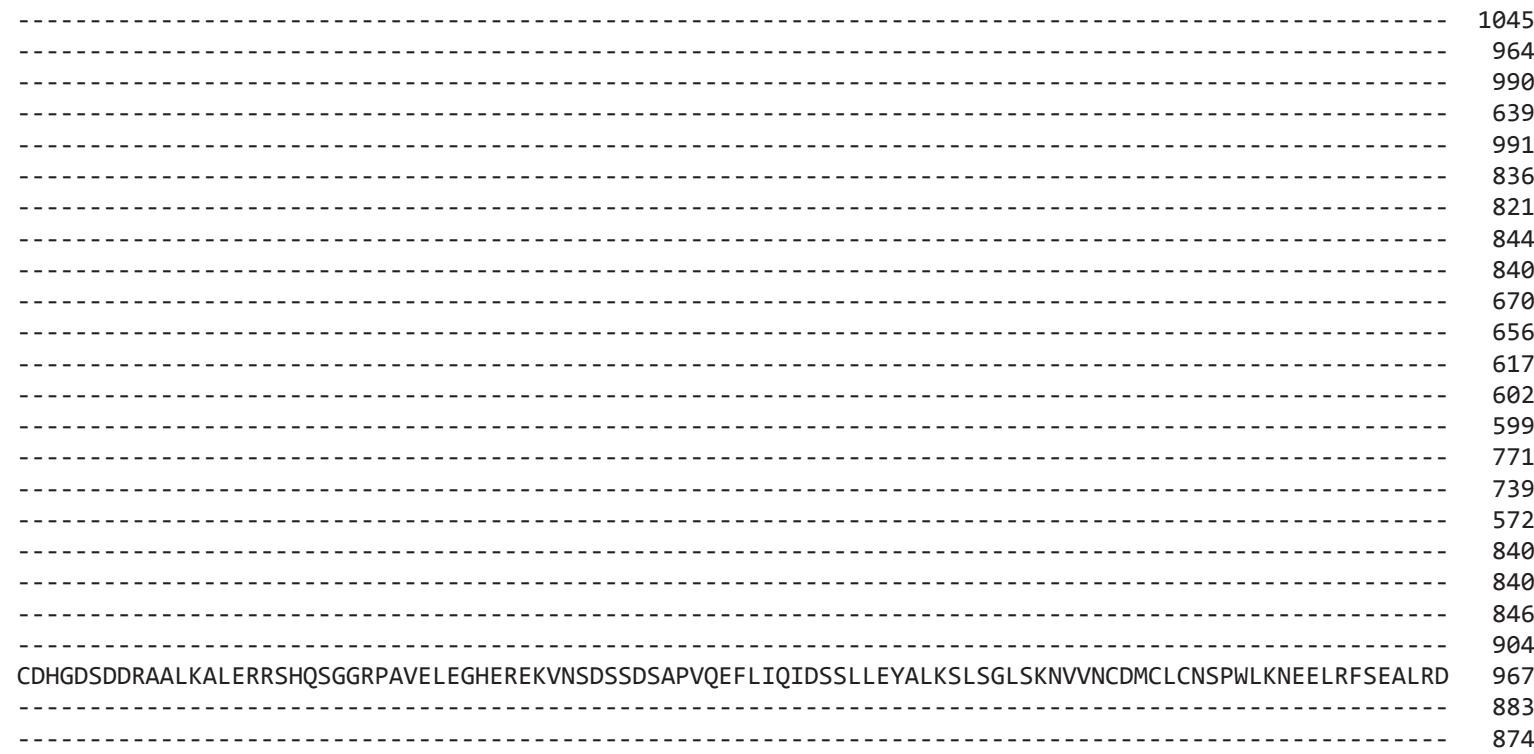

SdRV1:AYE54584

CLBV:NP_624333.1

CLBV:AFA43536.1

AVCaV:YP 008997790.1

CPrV:AKNÖ8994.1

ACLSV:NP 040551.1

GPGV:YP_-004732978.2

CtChV-1:YP_009103999.1

CtChV-2:YP_009103996.1

DiVA:YP_006905850.1

ObRV1:YP_009408144.1

CVA:NP_620106.1

ASGV:NP_044335.1

PVT:YP_002019748.1

PrVT:YP_009051684.1

GVA:NP_619662.1

GVE:YP 002117775.1

GCLV:YP_004936159.1

CVNV:YP_001430021.1

PhIVB:YP 001552317.1

APV1:YP_009094347.1

ASPV:NP_604464.1

CTLaV:YP 009046478.1

CRMaV:YP_007761581.1

SdRV1: AYE54584

CLBV:NP 624333.1

CLBV:AFA43536.1

AVCaV:YP_008997790.1

CPrV: AKN08994.1

ACLSV:NP_040551.1

GPGV:YP_004732978.2

CtChV-1:YP_009103999.1

CtChV-2:YP_009103996.1

DiVA:YP 006905850.1

ObRV1:YP_009408144.1

CVA:NP_620106.1

ASGV:NP 044335.1

PVT:YP_002019748.1

PrVT:YP 009051684.1

GVA:NP 619662.1

GVE:YP_002117775.1

GCLV:YP 004936159.1

CVNV:YP_001430021.1

PhIVB:YP_001552317.1

APV1:YP 009094347.1

ASPV:NP_604464.1

CTLaV:YP_009046478.1

CRMaV:YP_007761581.1

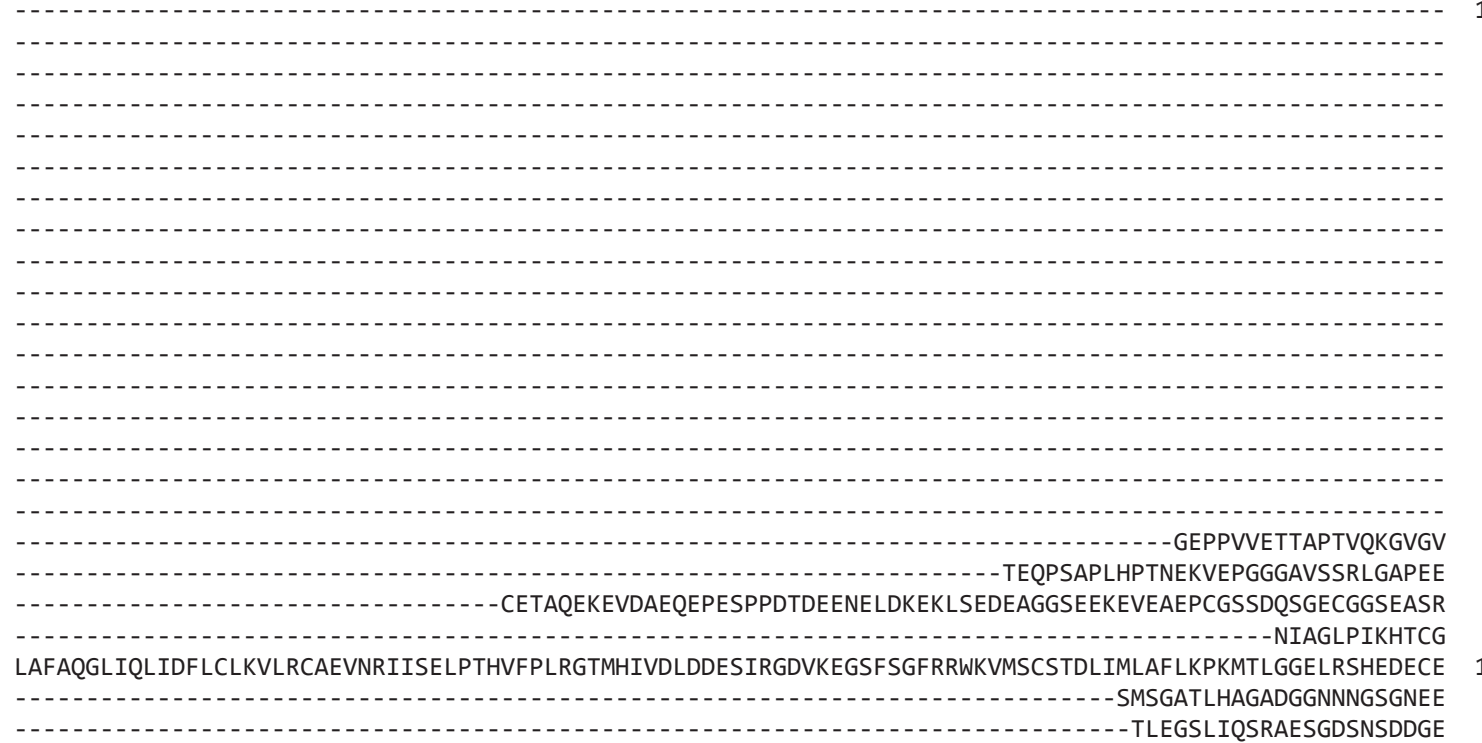

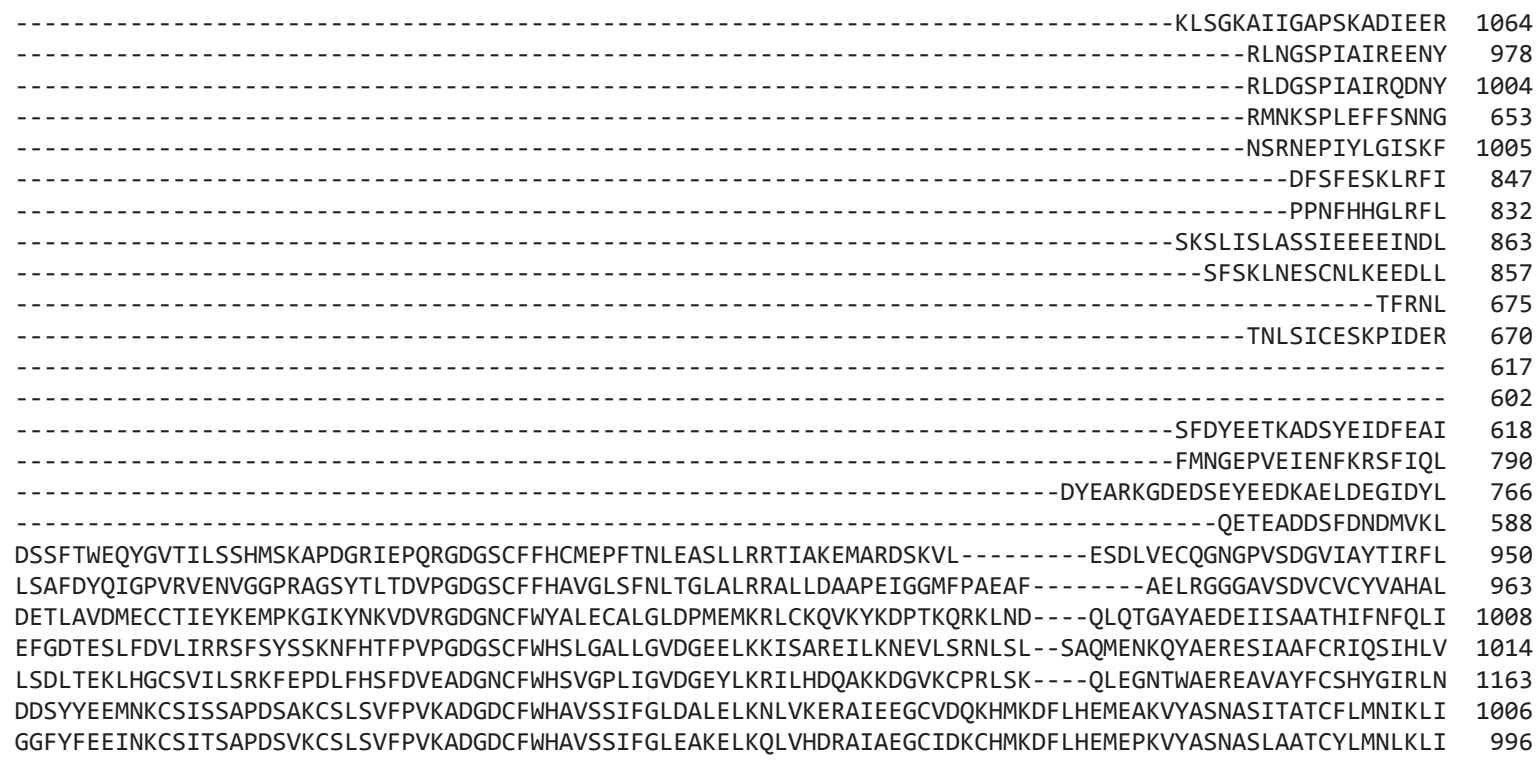


SdRV1: AYE54584 CLBV:NP_624333.1 CLBV:AFÄ43536.1 AVCaV:YP_008997790.1 CPrV:AKN08994.1 ACLSV:NP_040551.1 GPGV:YP_004732978.2 CtChV-1:YP_009103999.1 CtChV-2:YP_009103996.1 DiVA:YP_006905850.1 ObRV1:YP 009408144.1 CVA:NP_620106.1 ASGV:NP 044335.1 PVT:YP_ō02019748.1 PrVT:YP_009051684.1 GVA:NP_619662.1 GVE:YP_002117775.1 GCLV:YP 004936159.1 CVNV:YP 001430021.1

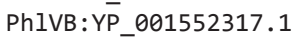
APV1:YP_0̄09094347.1 ASPV:NP 604464.1 CTLaV:YP_009046478.1 CRMaV:YP_007761581.1

SdRV1: AYE54584 CLBV:NP_624333.1 CLBV:AFA 43536.1 AVCaV:YP 008997790.1 CPrV:AKNÖ8994.1 ACLSV:NP 040551.1 GPGV:YP_004732978.2 CtChV-1:YP_009103999.1 CtChV-2:YP_009103996.1 DiVA:YP_006̄ 905850.1 ObRV1:YP_009408144.1 CVA:NP 620106.1 ASGV:NP_044335.1 PVT:YP $\overline{0} 02019748.1$

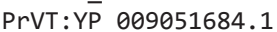
GVA:NP_б- 19662.1 GVE:YP 002117775.1 GCLV:YP_004936159.1 CVNV:YP 001430021.1 PhIVB:YP 001552317.1 APV1:YP_009094347.1 ASPV:NP_604464.1 CTLaV:YP 009046478.1 CRMaV:YP_007761581.1

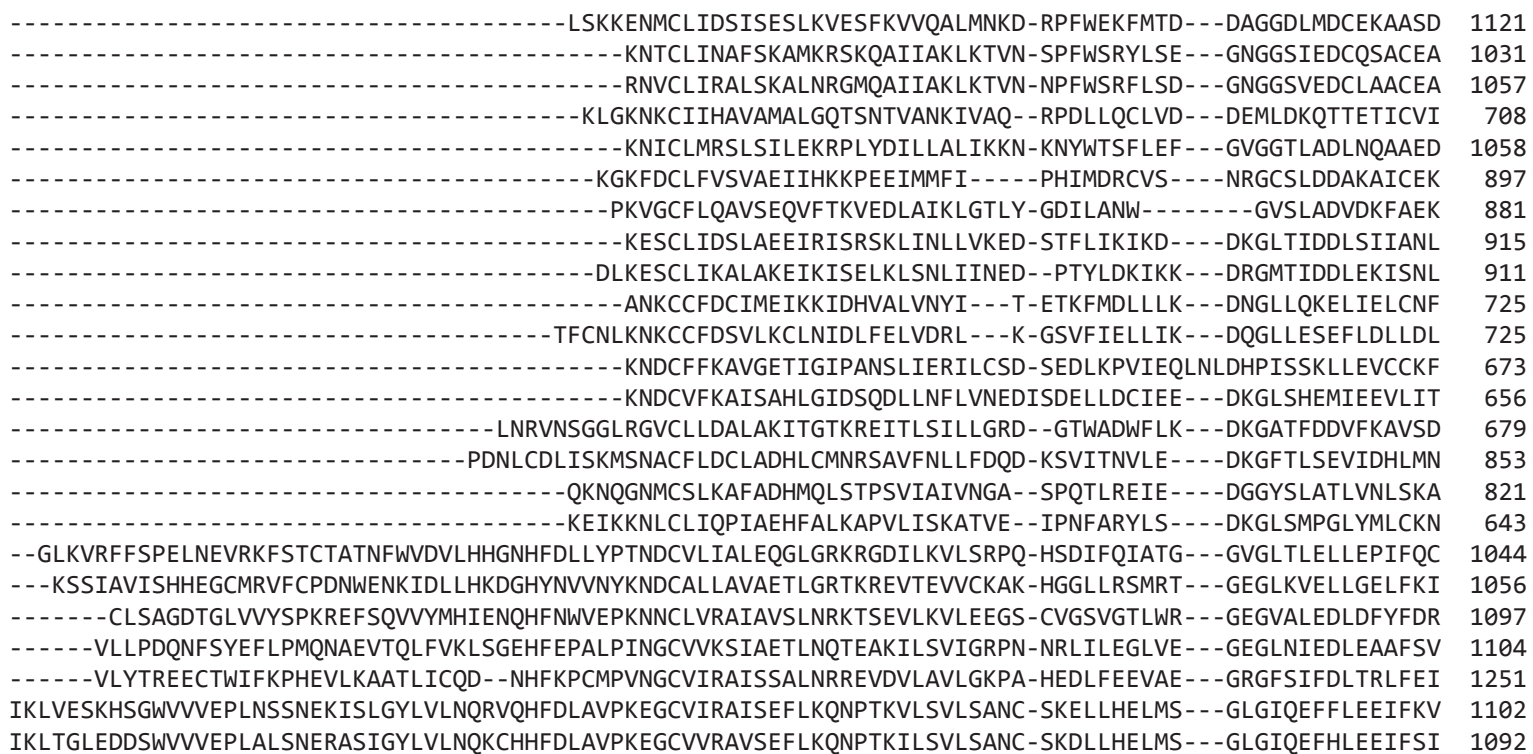

LLLNLEVHC -Q-DRINVFKFGQTIVKIT - - - LTNGHFSK - - LEEIKELPRSLVSQFKGKTRIDVVEGLVESLESE - - - - - -SH- -YNLFNFEANMSF LDVTVDLNV-N-GKCVVLGKGALRISMA- - - LRNNHFSV - - - INAAQLMERTFVSHLLEKGNVNVLEGFDAMLSGDV - - - - GAAG- -VNKIQFAANFEF LGITVDLFV-D-GKCLVLGEGAVRVSLA - - LKDNHFSV - - -VEEHRSIQRTFVSHLAKKSNLRVMDGLDEMLQSEM- - - - - STG- -VNCVQFIADFEH MNLHATIVNEDEGETMELNPEGLIKSSFS - -VLDEHMMVLSDIPNCRSKKGIDICMSPDLANSNCAANYEVTCQN- - - - - - - - - LQVIQYQADHER LSFRFELYM-N-EKWIAGGNRGPIYRLN - - -LSDDHFSV--- - - HRELSGNVEDTQLNFSKAKSKQSNFSSSDDDNSFDLDSIEH--VNKSLFEPLNDA YEIKIECEG-D-CGLVECGTSGLSIGRML - - LRGNHFSV - - -ASVRRSSMDSLANSSKEIKSDGVLDHVTFNFHKRLKLVEPD- - - - LTNADIKVDSSR LGIRLVLTN-G -FEVIRAGVEGPEVSMS- - -FSNGHFRS - - -NSNSRMNKGKGVESFPKEFARKVSDEMSNDANPQIIQNLNEVYGVFLNQTIFKLDPKR LNLSVRVLI-D-GQWSYFGVKESNYRLISLKLIKRHFSV - - -YNGEVLKLALGDESSDKLIKLSDSGFYNSFLNKI- - - - DPRNKFVNKFDRMINFER LCLKVRVLI-D-GQWQYFGVKESNFRGVSLRLSGSHFDA- - -YYKESIKIALGDEVSEKKVQKSADGAFFEGFLQRIDPKNEF - - - - VNRFERVIDLDR LNIKVNIIN-Q-SGTRLIYENDNDNTLI - - LTERHCKL - - -VKTESISDWLLDDN - - - - - KDFLDVTGV - - - - LGLNENVMN-A-AGFLVKECTESSGIFI - - -LSSNHCRF - - - ISKEECGDWFNKVK - - - - - - - GGLISLPGV- - - - - - - NYLLKDINCIKR LGYRVHIYY-G-DSIIKLNDDINMHAIHIGGKPGHLFCI - - NQERSKIPKDSQIKVPEVGPQSFIGSIFSKTYGS - - - - - GSSAPIHLGQIDITK KGLSMVYTS-DFKEMAVLNRKYGVNGKMYCTIKGNHCEL- - - - - -SSKECFIRLLKEGGEAQMSNENLNADSL - - - - - - - FDLGRFVHNRDR LDLNCTICT -KEGSFNAHVNRNYKHNFLY - LFDEHVSL - - ERPKVMLFEQVRHQKIDFL - - - - GAFEKCPG - - - - - - -

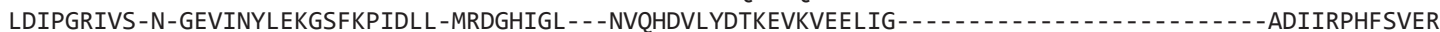

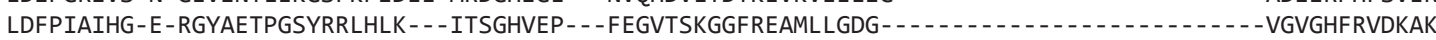

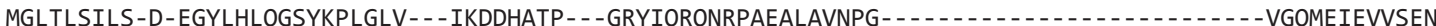
FQIDARVLC-G-SEVFTYPTSGTFALDFE - - LADGHLSF - - -KSARRKETVSSCLKVVEASP - - - HGRLVLNCA - - - - - - - - - GTGVLFEICSNT FSIKALVKC - N-GQLHRLNPEGKILGHYR - - LEDGHIQV - - -DKGLIKQLGSAPVKVIEHP - - -VHGSGMVLIAAV- - - - - - - - - ATQLSFEINAEI FGINAMVEF-E -GKAYNFNETGHLPAAFT - - LKDGHIEF - - - - - IGRNSPSTVPMMRGRVNTLTVSPNSLLIIKAA- - - - - - - - - -GSTIEFRPDYSR FGICARVST-E -RGVFTLNKEGNLHANYE - - LKSGHIMY - - LKKASSSQFAPTNPIQNFNGQ - - - GPEIFLRGI - - - - - - - - VSEVDYKPSWGR

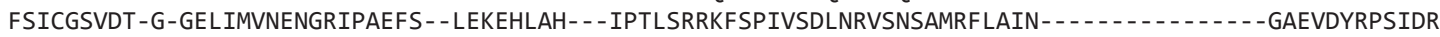
FDICAEVSD-G-EKSRILNANGSRSAKFT - -VEQDHFSF - - -SPGVKASTNLGSFKAPSGGQTIPIEQYETFLRGN- - - - - - - - ANVIPFTPSLIA FDICAEVSD-G-ASSRVLNKKGSRSAKFI - -VDKDHFSF - - - CPGTKASTNLGVFKAPSGCPMIAIEKYDEFLRSS- - - - - - - ANVVPFTPSLPL
1203 1116 1141 793 1145 985 973 1004 1001 794 794 759 734 754 921 886 708 1120 1134 1176 1181 1328 1183 1173
SdRV1: AYE54584 CLBV:NP 624333.1 CLBV:AFA43536.1 AVCaV:YP_008997790.1 CPrV:AKN08994.1 ACLSV:NP_040551.1 GPGV:YP_004732978.2 CtChV-1:YP_009103999.1 CtChV-2:YP_009103996.1 DiVA:YP 006 905850.1 ObRV1:YP_009408144.1 CVA:NP_620106.1 ASGV:NP 044335.1 PVT:YP_002019748.1 PrVT:YP_009051684.1 GVA:NP 619662.1 GVE:YP_002117775.1 GCLV:YP̄_004936159.1 CVNV:YP_001430021.1 PhIVB:YP_001552317.1 APV1:YP 009094347.1 ASPV:NP_604464.1 CTLaV:YP_o09046478.1 CRMaV:YP_007761581.1
AGPLMNSFLNRSTG-- - - - ICLGSVINNGGKYF- - - - EDVFSLDESSYRICS- - - - - - - - - - AIHALNGFAGSGKSKVMQNWLSKRRC

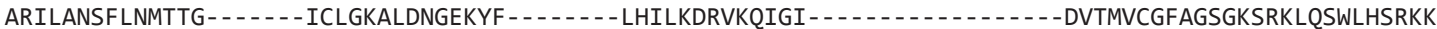

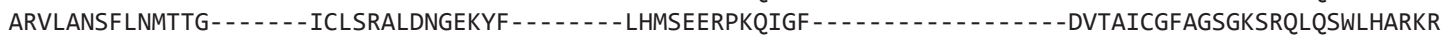

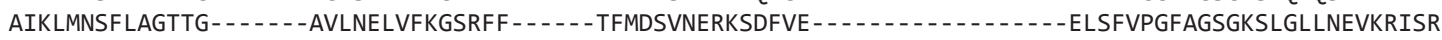
AELLRQSFLNRTTG - - - - KILSDAFGENGAHLR - - - - RIRIVKSDDPFPE - AGKLLKSLMDGMTG--.- - - IVSHNSTHEGWRMIKGINSTSEMRSFMNMVRGQIEEPKSDLFDKVQELNFMKVKIYGIFGFAGSGKSHAIQNLIQTEFK AQRLLKSLLDGSTG-- - - - -VHCNSSLKEGWKMI - - - - PNAKSSEFVTKNYIN--SSDIWKGSALWN-AKINISGIFGFAGSGKSHGIQRLLNEKFS AALLIHSFLRGSTG - - - - - VVTSSGFNNGVDYF - -SGRKRNIDPESFRDPKFLK - - - - - EMTKGDAVVKGAVILGFAGCGKSRPVQMALDSMDS

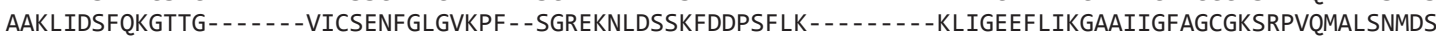
SKKLYDSLSKGTSG - - - - - VFFNMIKKKNDESE- - - - - KKKDKNRVIEMMNFFFEDEINEKRKLTGRSEPIYGFFGFAGSGKSREIQNYINTNYN AGRLFKSLSRGNTG - - - - - - ILFNSIKKKSDESE - - - - - - KRKDKNRVIEFLNLIFDNDDDHEVEIVKRDEPIYGFFGFAGSGKSRAIQGFINSEFN ALVLVSAFESMNLGVRVDRKAILEGQLISNGFLAF - - - - - LKRKNNEGHKVIK- - - - - - IQSLPVYPFIGFAGSGKSFGLTEKLINGDC

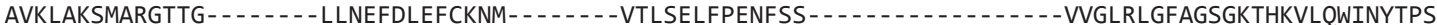
GSLLASALKDNLTG- - - - VISSKFNWDPKCEF - - - - - ARVLVKSMMEGMTG- - - - - VILNRFKHAFNELL - - - - ADRLAQSFYNGNTG- - - - - VLLGKYNKGKMHTG - - - - - - -

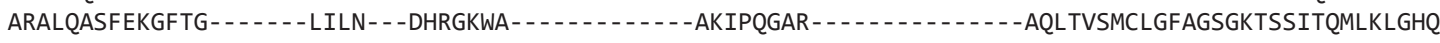

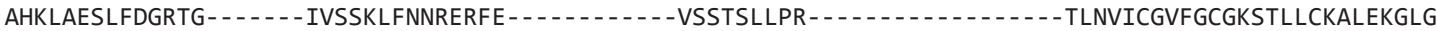
GDKLAECLVSGRTG - - - - - VISSQLFNGRQKLT - - - - AKCLADCFHTGCTG - . - - VMNSKIFNNSEHLL - _ -

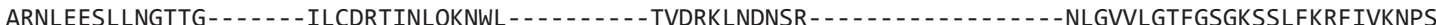
ASTLLDSFEIGATG - - - - VLCQGIKEAQKDLA - - - - -

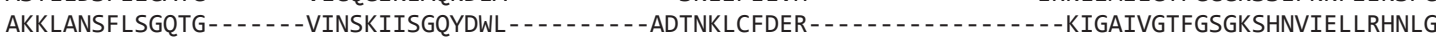

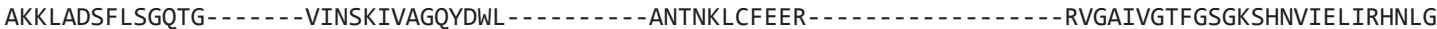
$* \quad * * *$ : 
SdRV1: AYE54584 CLBV:NP_624333.1

CLBV: AFA43536.1 AVCaV:YP_008997790.1 CPrV:AKN08994.1 ACLSV:NP_040551.1 GPGV:YP_o 04732978.2 CtChV-1:YP_009103999.1 CtChV-2:YP_009103996.1 DiVA:YP_006905850.1 ObRV1:YP 009408144.1 CVA:NP_620106.1 ASGV:NP 044335.1 PVT:YP_002019748.1 PrVT:YP_009051684.1 GVA:NP 619662.1 GVE:YP_002117775.1 GCLV:YP 004936159.1 CVNV:YP_001430021.1 PhIVB:YP_001552317.1 APV1:YP_ö09094347.1 ASPV:NP_604464.1 CTLaV:YP_009046478.1 CRMaV:YP 007761581.1

SdRV1:AYE54584 CLBV:NP_624333.1 CLBV:AFA-43536.1 AVCaV:YP 008997790.1 CPrV:AKNO8994.1 ACLSV:NP 040551.1 GPGV:YP $\overline{0} 04732978.2$ CtChV-1:YP_009103999.1 CtChV-2:YP 009103996.1 DiVA:YP_006905850.1 ObRV1:YP_009408144.1 CVA:NP 620106.1 ASGV:NP_044335.1 PVT:YP 002019748.1 PrVT:Y $\bar{P} 009051684.1$ GVA:NP_619662.1 GVE:YP 002117775.1 GCLV:YP_004936159.1 CVNV:YP_001430021.1 PhIVB:YP_001552317.1 APV1:YP_o09094347.1 ASPV:NP_604464.1 CTLaV:YP 009046478.1 CRMaV:YP_007761581.1

\section{SdRV1: AYE54584} CLBV:NP_624333.1 CLBV:AFA43536.1 AVCaV:YP_008997790.1 CPrV: AKN08994.1 ACLSV:NP_040551.1 GPGV:YP_004732978.2

CtChV-1:YP_009103999.1 CtChV-2:YP_009103996.1 DiVA:YP $00 \overline{6} 905850.1$ ObRV1:YP_009408144.1 CVA:NP_620106.1 ASGV:NP 044335.1 PVT:YP_002019748.1 PrVT:YP_009051684.1 GVA:NP 619662.1 GVE:YP_002117775.1 GCLV:YP 004936159.1 CVNV:YP_001430021.1 PhIVB:YP_001552317.1 APV1:YP 009094347.1 ASPV:NP_604464.1 CTLaV:YP_009046478.1 CRMaV:YP_007761581.1

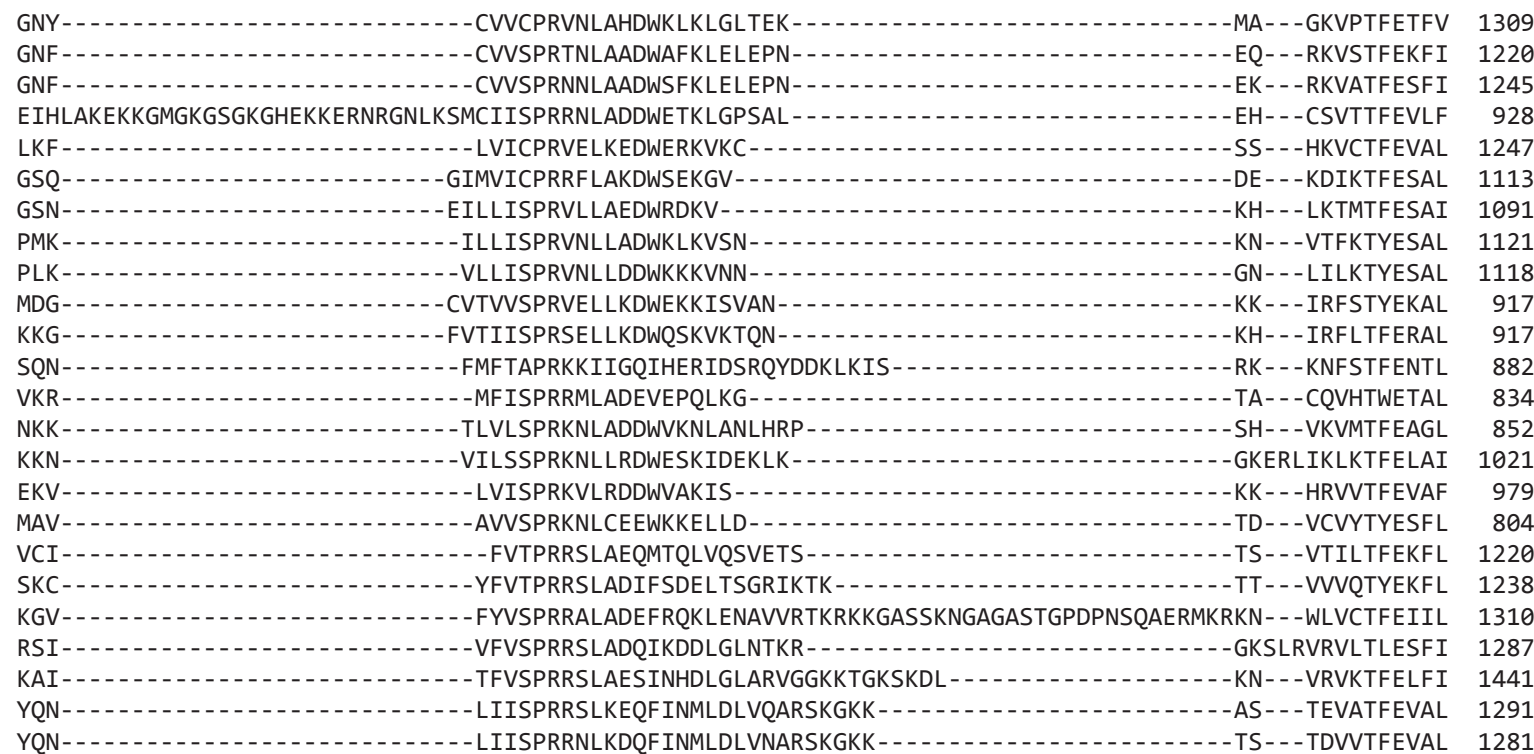

1309

247

1113

1091

1121

1118
1245
928

-

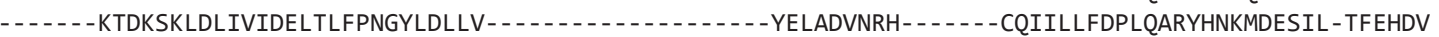
- - KMDKSKLDMIVLDELTLFPNGYLDLLI- - - - - - CHLILLFDPLQARYHNKMDEAVL-NFEHDV - - KASISKIKLIVVDELTLFPNGYIDLLI- - - - - - CKLILIFDPLQARYDSAQDRAIL-GSEHDV LQNLSRVELIVIDELGLFPRGYLDLMI- - - - - - FKLRTEKNFK- - - GKVMLLFDPLQARYHSDSDERFL-HEIHEC

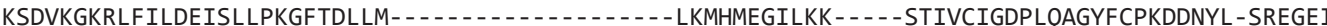
- - - KGCLAGYKWIILDEVTLFPNGYLDLLV- - - - - - - - LKLAHYNEINL - - - KHITLVGDPLQANYFNERDCNLL-GSVKMV 作 KENFAEFSMIVIDEFLLVPRGYLDVAA . . . . . . . . . . . FKSKMDCKVCKSKPRIPKFLLLGDPLQAGYYNALDDHLI-PEKSEM TLSYYEDELVVVDEIGLLPPGYISLLSLVTAFRVNKISHN - . - . - - IRLSKRNYSKYVENQSSRLVLLGDHLQGRYYNESDFRSL-SQPDEI -TVTYQESELIVIDEIGLMPPGYMSLLNIITSIKFEEVSNN- - - - - - FRLSRRNFRNFIGSPRSRLVVLGDHLQCRYYNDSDVRSL-DPKDEI - - - - LSLVNKPLVIMDECSLNPPGF IDLVLIKSLDSIIRKSNKDFDHFFSSSVLSEGIIANVASPIACIAVTGDTLQSSFYSESCGKLM-QYKNDI -KKIDGTFMEVFVDEIGLYPPGYLTLLQMCAFRKIVKGQSENFLKGKLLELSKTC- - - - . - LNIRCFGDPLQLRYYSAEDTNLL-DKTHDI

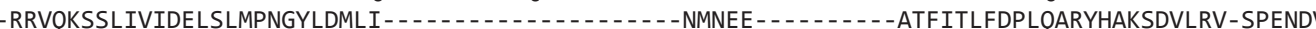
SAITRMVKKEQDGKLTVIIDEATLLPGGYLDLVN-_... -MDDGCKDIVI-DEIGLLPPGYIDLVI-

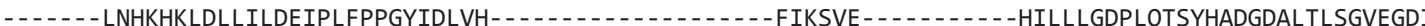

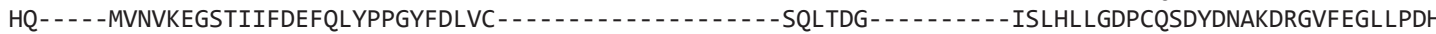

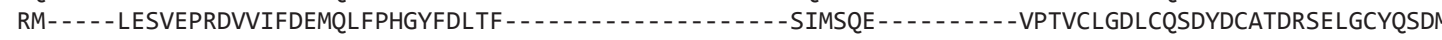
KK - . - IHLVKPGMALILDEIOLYPPGYLDLIC . . . K- - - AVFTFKAASVVLDEVQLYPPGYLDLVM - . LH----LDSIKEGHTVVIDEIQLFPPGYIDLII - - K- - - KTGMLKKVRIFIDETQLLPPGYLDLVC - .

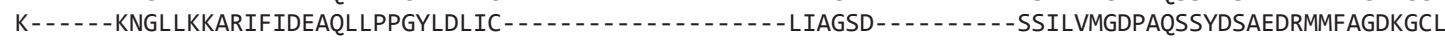

DRM - - ISGQR

DRL - IGGQN -

DRL--IGGQD-1 -

DLI--LGDSE-

DRI--TSGAK--

KRL--FKGG-1-

DSV--FKD-1-1

SSL--EIDY-1

ETL-DEIRK-1-

DFI--MMNEE-1-1-1-10-10

VFL- $M E N E E-1.0$.

KTLCALSHTR-1-1

DLM--IKTIK-_.

DRI-1 - KV -

TPI---FGQE--

FNR--VRGK - .

FRR-- LQAKKSGICPCGMHFKPSRYMGPNVEFDFDEADKLKGREAIFFSRGGEGYKYNGGDHKSSGWVGELDQIIDACNINADSFDHCLVQRYIPGGSIK 964

QRI--LQGIQ-

QRL--LQSAK-

DSV--LEGAE-

FEV--LSGKK-1._-

MRI--LSGRS-1

DVL--LDNKR-1-1

DRL--LEGKK-_. 


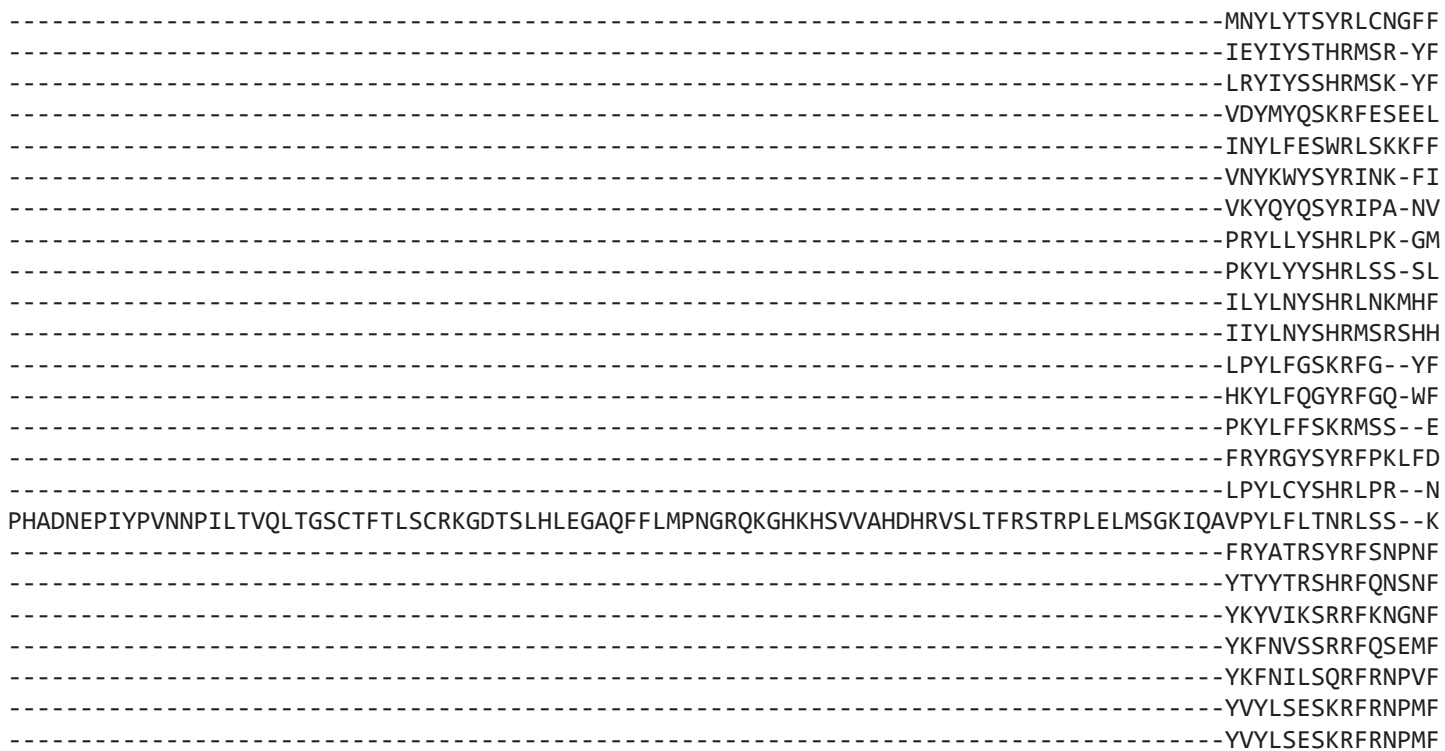
MNYLYTSYRLCNGFF RYIYSSHRMSK-YF NYKWYSYRINK-FI PKYLYYSHRLSS-SL IIYLNYSHRMSRSHH LPYLFGSKRFG--YF 作 FRYRGYSYRFPKLFD PPYLFLTNRLSS- $-K$ (T) YKYVIKSRRFKNGNF KFNVSSRRFQSEMF

KDVFEKLOLPIKO- - - - - - GDEERRFLYHNPFRIOEDSSETIK-VDVLIVESOLEKK - - - -TFGSAIKCMTFGESOGLTF-DHVCILLSESSANSC NRFF-DVPCFNQADR - - - - - - TEEQRLWIFDDVYSIPSICSDRQEPCDVLLVESDLEKK--.--AFSPIINVMTFGESQGLTF-NHVCILLSESSAASN NRFF-DVPCFNQAET-- - - - TKEQRLWILDDVYSITSVCIDQGEPCDVLLVESDLEKK- - - -AFSPVINVMTFGESQGLTF-NHVCILLSESSAASN FNLF-EDLKKNEVDAESRETGKGAKFRPRMYTNLLTMKVEEENOGNPIDVLLVGSFDEAG - . - - LFASSIKTMTFGESOGLTV-DHAAILLSENSALSD GNFFVDIELRNSG- - - - - - -SVNYELDFFDNHIVAANEAKKRGFPIDLILVASRDEKN-----SFAGKVNVLTFGEAQGLTV-KHSCIVLSEYAEKQD AKKL -AIETMNDFI - - . - - - GIDEOSSIYKDMPSAHHFMEKKGNHIEVILVASMVEKE- - - - LYSNYGNVMTFGESOGLTF-NCGVIVLSEEAKLCS AGRF-DVWDKNRHE- . - . . - _ PIDCHGTFYSDLSSAKLHAKRCNOKIDVVLVASELEKK - . - -YYFSNOCKCITYGESOGLTF-DYGLISLSEESRLCS KSMM-DINMLGSF - - - - - - - EGETKWKLYNSAAAAFSEKA - . - -FDVILVAGRQEK- - - - TFFGNFTVMTFGESQGLTF-NKVCIALTEDSLLAS GGIL-DVPMLGPI - - - - - - - - NELNQLNLYNSAAAAFSERA- . - - FDVILVAGRQEK - . - . - SFFFSNYTVMTFGESQGLTF-DKVAIALSEDTLLCS YKP--GVEMLGE - - - . - . - - DENIISRRFSNVFSAKKTIP - . - -EAQLLVASRDEQ- - - - -VRFKELDAKTFGESQGLTF-DEIIIVLSPPAVNCS YKP--GVVFLND- - - - - - - ADSVPTKRFLNTLVAKKSIP - - - -NAQVLVASHDEQ- - - - IRFRDLGAKTFGESQGLTF-DESIIVLSPPSTNCS TGFL-KLGYYN- - . - _. - - OMESKAFTIDNMETLOKAIGTSMDKFGVLVTSRADKSD- . - - FELDFPNVCTINEAOGSTF-NSVILIVTRDFFSNP

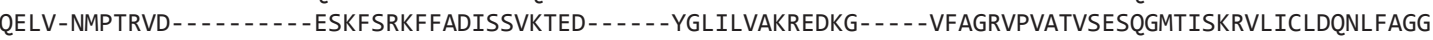
LDFF-DVRCSSDQK - - - - - - KWELHGKQYREPAALFRDIKGQE --FTILSPSFETAREMSKYADIKDGCKSMTFGESQGLTV-NKAVIVVDQDLVATS LEDF-EYGHGD - - - - - - - - - -VDPNHMRVFAOPOAVREAIK - - - -RPVFLCPSDDKRS - - - -ELSNFGEAYTFGTSOGLTF-DFVCISIDMDGSVTS CKLF-EIECM - - - - - - GAESEKRVVYRSNRLKDEPT - - - - - ICATRAMKE - - --EKGSG - WYTVSETQGLSF-KSCLIYLDEHWAKKE

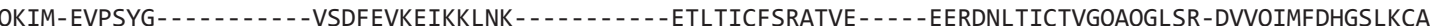
VG---RLPCA-_.....--ISNTNEDDFEDFEILEGIEQVQEIDVECYLVSSFIEKQAVRA-LVGLDKVVQTFGESTGLTY-DCVAVVVSEASKLAS AG - - -RLPCQFQPEYF- - - - - TNHEDFTILHGFESLNDIAG - - - - LDCILVSSFNEKTAVKALTFGR-VSVQTFGESTGLTF-NSGAIFISEVSKLAS QG---RLPCEFGTQMEG-----QATEEHLLYSGLEHLHVIPQEF---SKVFLVSSFEEKKIVEAHFPGSNPTVLTFGESTGLNF-KYGTIIITNVSAHTS VG - - -RLPCRMDTKAM- - - - - TENENFHWLESIESAAEVSNTE - - YDVVLVSSFEEKKIVWA-HLGRDLEVLTFGESTGLTF-NRGIILISHESTLTS YG - - -RLPCNLNKTRLT - - - - - LDEEEYTLWDSIQEFFSMMGRKD - - CPVVLVSSFEEKKIVAA-HLGLKMKCITYGESTGLNF -QKGAILVTYESALTS LG - - -RLPCTFDOSRMT - - - - LEKEEYAVFSSFFRDFKNDYLSPK - - IKTFLVSSFTEKTVVKA-NMGRNVLVYTFGKSTGMNF -DYVCVLLTODSMLVD VG - - -RLPCTFDSSRLT - - - - LEKEEYAVFDSFKAFKADYLSPK - IKTFLVSSFTEKTVVKA-NMGRNVSIFTFGESTGMNF-DYVCVLLTQDSMLVD ASPV:NP 604464.1 CTLaV:YP 009046478.1 CRMaV:YP_007761581.1

\section{SdRV1: AYE54584} CLBV:NP_624333.1 CLBV:AFA43536.1 AVCaV:YP_008997790.1 CPrV:AKN08994.1 ACLSV:NP_040551.1 GPGV:YP_004732978.2 CtChV-1:YP_009103999.1 CtChV-2:YP_009103996.1 DiVA:YP_006905850.1 ObRV1:YP_009408144.1 CVA:NP_620106.1 ASGV:NP 044335.1 PVT:YP_ō02019748.1 PrVT:YP_009051684.1 GVA:NP 619662.1 GVE:YP_002117775.1 GCLV:YP_004936159.1 CVNV:YP_001430021.1 PhIVB:YP_001552317.1 APV1:YP 009094347.1 ASPV:NP_604464.1 CTLaV:YP 009046478.1 CRMaV:YP_007761581.1
EFRWMVALTRAKK - -RLSFCCTHLGGIEDFTTNCKSQLFKSFFSRQT - - IKVDFLRSLCQAKMNMVKKE - IGGKIDEVDREERLSGDPFLKPFIFLGK EFRWMVALTRART--RFSLCSTFLGGIEEFKVKRKESLITSILOG-- - - EKITFNRLNLMLKCNLIRRE-KENGCRDEVDREERLEGDPFLKPFIFLGQ EFRWMVALTRAKT - RLSFCSTFLGGMDEFKIKRGESLVTSILEG- - - - KQITFERSNMMVKCNLIKQE-KKNGCSDEVDREERLEGDPFLKPFIFLGH DHRWLVALTRARK - -KVTFLCLHLSGLNGFLSTMENRLVAAVINKGLVTKKRLSSMVRAKLNYVKFKGLA- - - - GKDEVDREDRLEGDLFLKGVIFLGQ DYRWVVALTRAKE - -KISFITSHRSGLTGFMSSMIGRPIHAFLTG- - - - LPFTSNRMNWMVNCELVECH-RATGGRDEVDREDRLEGDPFLKPFVFLGQ DAHIMVAITRFRR - -GFCFALGSKGSKEDYMRSMKSGLLQRICSGVG - - ASKEFILGSSSVNLILSEKDIAKGAGIDEMDREARLEGDVWLKSMIYLGK DNHIYVALTRFKK --GFGFFQNFRGDLGTFKSNLGSKLLGRYINLRD---NLKPFMMOMLDINLDFMDDR - - -NQVGAGIEMENKMSGDPWLKGLLDLQA DNHMMVGLTRAKE - - TINFIKGFGYPLNEYVKKAGNKLIGKVLLGKVIKRAELENMSG - -MEDVTFITEP - - - - - - PTFGGHEDKVOGDPWMKSLLTLTO DNHIVVALTRARK - -QISLIKCFGYDEKEFFKRAGTKLIGKVLNKKKIKRVQLENM- - LALEDLKLISSE - - - - - PKFGTQEERTEGDPWMKGLLTHIQ INMWNVAMTRARK - -GVHFALNGFDTVDDFINRVKGTPVNAMILGSP - - -FEIHRTPGGKDKEIKIIKVC - - RLGMSNEDVEMKLMGDPFLKSIIPSLD LFMWNVAMSRSIK - -GVHFALNGFDSIDDFLNRVVKGTPVAAMILGMK - - -FDIHAQPMSTPEDCKIICSD - - -RLCLSSSDVENKLEGDPFLKSIIPSMD IESIIVAITRHQKNLLIYFPAAIQGEMDFLSRRF - - PIHSNVVLKNF - - - SVLDNLIKDKLNPFQLIQED - - - - - PFGHDFEVKLEGDPFLKSELSLVN ANAAIVAITRSKV- - GFDFILK - GNSLKEVORMAOKTIWOFIIEGKS - - - IPMERIVNMNPGASFYESPL - - - - - DVGNSSIODKASNDLFIMPFINLAE VLHWIVALTRSRQ- - GFVILVHKVFDMKTLIQPVQNSIIGLVLRGVKVQENIF INTAGKCLSEAEIVEEL - -ETFKRTEED- EDLLEGDPWLKGQLFLCQ DFHWMVALTRARR - -GFCFLTCASTSMRTFMDNNRAKLIGKVLKKEQISKKFWWNLGGRALEGARAVKKDEFSKLGKTREEFEESLEGDPWLKGMLNYLE DEDVMVALTRSRGEIGIHVTPALK - - -KKLITNAKSTLLKKVLKGETYRRSEIVAMVRKHIPETTVLFEE - - -SRLAETVDYEARLAGDPYLKSLLALYD DETIIIALTRARK - - AVHLFYKIG - - KTDLKNCSSPILRAFISNGKIPEKLLVDKVRGKLGDCRLLTEN - -VFIGADSATIGDHLAGDPGLKAMLLILE ERRWITALTRARK - -RVTFITNLGCSKHLIAEIFSNRALGRFLSCT - - - ASIDNLRCLLPGEPNFVEEL -VPTIGANLGVVEEKVSGDPWLKTMLFLGQ EQRWLTALSRFRM - -NLTFVSDLGCDSSMLAEVFSGRVLGRFLSGK - - - ANVCDLRGLLAGSPDLQEDF -PTTVGKNQGLVEEKVVGDPWLKAMLFLGQ EKRWVTALSRFSE - - NICFVNLVVNLSWSELARMYATRVLGRFLGKR - - - AKLSDLLEHLPGVAVFTDSY-DENIGKDEGVREEKVQGDPWLKGMVDLFQ ERRWITALSRFRL - -NIIFVNLVGNCLEDACOVFHDRTLDRFLTKR-- - -ATIANIVDQLPGLPELTNDF-GDKVGRSEGVMEAKLSGDPWLKTEIDLLQ DRRWWTALSRFSH - DIHFINGMGVTWDNAITHFVGKPLHKFFTKR - - - ACNDDI IDLLPGRPELIEGF - QSQVGADEGVREAKLVGDPWLKTKIFLGQ ERRWVVALSRAKI - -NMSFVNLSGLSLPEFCTQMVGGVVHKFFTGT - - - -ATFNDLRSLLPGDPIFSKKF - -QRLGSDEVDREARLSGDPWLKTKVFLGQ ERRWVVALSRAKI- NISFINLSGLTLPEFCTQMMGGVVHKFFTST $----A$ .: :* 
SdRV1: AYE54584 CLBV:NP_624333.1 CLBV:AFA43536.1 AVCaV:YP_008997790.1 CPrV:AKN08994.1 ACLSV:NP_040551.1 GPGV:YP_ō04732978.2 CtChV-1:YP_009103999.1 CtChV-2:YP_009103996.1 DiVA:YP_006905850.1 ObRV1:YP 009408144.1 CVA:NP_620106.1 ASGV:NP 044335.1 PVT:YP_ō02019748.1 PrVT:YP_009051684.1 GVA:NP_619662.1 GVE:YP_002117775.1 GCLV:YP_004936159.1 CVNV:YP 001430021.1 PhIVB:YP_001552317.1 APV1:YP_ō09094347.1 ASPV:NP_604464.1 CTLaV:YP_009046478.1 CRMaV:YP_007761581.1

SdRV1: AYE54584 CLBV:NP_624333.1 CLBV:AFA43536.1 AVCaV:YP 008997790.1 CPrV:AKN0̄8994.1 ACLSV:NP 040551.1 GPGV:YP_ö04732978.2 CtChV-1:YP_009103999.1 CtChV-2:YP 009103996.1 DiVA:YP_006̄905850.1 ObRV1:YP_009408144.1 CVA:NP 620106.1 ASGV:NP 044335.1 PVT:YP $\overline{0} 02019748.1$

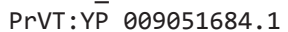
GVA:NP_-619662.1 GVE:YP_002117775.1 GCLV:YP_004936159.1 CVNV:YP_001430021.1 PhIVB:YP 001552317.1 APV1:YP_ō09094347.1 ASPV:NP_604464.1 CTLaV:YP 009046478.1 CRMaV:YP_007761581.1

SdRV1:AYE54584 CLBV:NP 624333.1 CLBV:AFA43536.1 AVCaV:YP_008997790.1 CPrV:AKN08994.1 ACLSV:NP_040551.1 GPGV:YP_0004732978.2 CtChV-1:YP_009103999.1 CtChV-2:YP_009103996.1 DiVA:YP 006̄905850.1 ObRV1:YP_009408144.1 CVA:NP_620106.1 ASGV:NP 044335.1 PVT:YP_ō02019748.1 PrVT:YP_009051684.1 GVA:NP 619662.1 GVE:YP_002117775.1 GCLV:YP̄ 004936159.1 CVNV:YP_001430021.1 PhIVB:YP_001552317.1 APV1:YP 009094347.1 ASPV:NP_604464.1 CTLaV:YP_009046478.1 CRMaV:YP_007761581.1
RVEDREIV-IDECDPEEVKCKTHLYLSEPNFAGAYNFDLIREKESREYRESM-MSTDQFCDNYNKKHPKACMRTVGPMRFKSIYPKHSSDDDMTFWMAVK RVEKDEDE - VEEVKIREPTCQTHLYITEPNFGLCYNFDFIREKEQREYREDM-LVTNQFCDSYDKVHINGKRETPGPLRFKAIYPKHSADDDMTFWMAVR RIQKSHDE - VGEIEVREPTCQTHLYITEPNFGLCYNFDFIREKEQREYREDM-LVTNQFCDSYDKVHINGKRETPGPLRFKAIYPKHSADDDMTFWMAVK RCEIMEPE - IVEPVMAKEDMKTHFFVCQENFAQCYNFDNIRAKELREFRIGH - RVTNQFIDNYEIVQHVQKKHTAGPLRFEAIYPRHCADDDVTFLMAVH RINSEEYE - IIEPEVIEPKGRVHLCISOENYALARNFDLIRAKEYREAKLMG - LETNOFCHDYNRVGAOGSRHVASPLRFESIFPRHRSDDDLTFWMAVK RYHMVEPL-GQVIKLTDDAIKCHIPVCSSQ-TLGPELDNIQAREYREFKGKN-GWSNQFREEAG-PNWKFPYKVNQAMSYEAVYPRHKMDDDLTFLAAIK VEEVEDMF - FEDLNIIEPTGKVHLPLASRN - - - DEFEKIRARESRELKKLDFDWSMQFEDCGV- - KIKRVLNGNLCENFSAVYPVHQACDEMTFLAAVK REDSOEVE - LIEPDIVESKMKVHINITDKSYALMIINDQLRAKENREFKSKD-SWSNQFKDNDQ - - NLNLETSTGPVNFEAIFPRHQTFDDVTFWMAVK EVIMEECE - PQMAKPDDVKMKVHVPITDKSFALTIINDQIRAREYREFKVGD-SWSTQFKDDNK - - NLKLESSTGPVNFESIFPRHTSFDDVTFWAAVK EGLSIEQE - YHDIICESPVPKIHLPIESIQGHVSYVSSMLKERGEREFKGDG - CMSEQFPDFWK - -TGEPGHYLSQSERFQSIFPKHQNSDSLTFLAAVK EGLCVHHE - YQDVNFELPTPKIHLPIESIQSHVAFVSSMIRNREFREFIGDG - EMSEQFPDFFK - -OSETGSFLSOAERFQAIFPKHSNGDSLTFFAAVK EIKLQQIE - ENSIESKENL - KTHLPI - SYSGLWNLEISEMRAREDREFKKFGVGWSKQFKDEPN - - QKDQVEDNCAMLPEAVFPRHFANDDLTFWSAVK EEVDPEEV-VGDVIOPVEWFKCHVPVFDTDPTLAEIFDKVAAKEKREFQSVL - GLSNOFLDMEK - - - NGCKIDILPFARONVFPHHOASDDVTFWAGVQ SVELDEVT - PEEPLRHESPPRTHLPL - PVEGLTPLLMSNVKAREDREFITPS - GWSKQFRDDKE - - - NVDWRNVSYADAFETIYPKHEASDDITLWAAIQ GDDANDPE - PEEPIRKDSPPRTHLMIAPVEHQFAEEMHLLRAREFREFRNSN - LWSEQFDDCRK - - - TRKVIHNRAETFEQIYPSHKNSDTLTFWAAIK EIEMEDIE - IEEPVTLEPT -KTHLALSTKMNELAPF - -DLKAKEHREOHTEA-GRTEOIDENGY - - - - - OGEVGDPMTHKALYLRHTSDDTATFMMSVK AEEMEPEI -FEEETVPETI-RTHLGVTT - -FANEQFAFGLKAKEEREHHIHGTGFSTQIRDNIA - - - - - SEFHPGPSAPSSIYLHHTAEDDVLFILSIK VADVADEI -DVDEALQIEPFKTHVARSNLEGVRALWHDKIRLKEHREKRMGY - LVSEQFTDMHS - -KNMGKKLTNAAERFETIYPKHKGSDTVTFIMGAR ETDEOEVA-PEVAEVALEVFKTHAPRCELEGVRARWHEKIOAKEFREKRMGY - LVSEQFTDEHS - KNNGKKLTNAAERFETIYPKHKNSDTVTFIMGAR IEDVEEEE - EQLEEMQTEWFKVHLPQAELESVRAKWVHKILAKEFREVRIGH-LVSEQFTDEHP - -REQGKQLTNAAERFEAIYPRHKANDTVTFMMAVK DEDQEMEE - LAEEVKHEPWFKTHLPLFELESIRASWVHRIMNREYREVRCGS - ETTTQFPDDHP - SGAKITLANAAERFEAIYPRHRGSDSVTFLMAVK NPDFEIEI -ADEVEAAEDWFKTHIPIMSLEAVRAQWVHKLISREDREFRIGD-ITTEQFTDDHS - KNRGQELTNAAERYEAIYPRHKGTDTATFLMAVK REVRPVEEPISVENLKDIKIKVHCPVGSMGATFAEVQSKLKVKEAREHRIDT - IVTEQFAEVHK - - GRGKILTAAPDNFEAIYPRHKAGDTATFVMAAR REEKIESIHVNDEGLKDIKVKVHCPIGSIGSTLADIQAGVRVKEAREFRIDN - LVTEQFSEVHK - - GKGKVLTAAPDNFEAIYPRHKAGDTATFVMAAR

KRLRFQSEEENRMKLSEAHLIGGILYQNFKEKFD-LDFSHDQGLFERCVN-EFEEKKLGKSQAVIKSHSNRSDTDWKINDIFLFMKNQLCTKFEKOYVDA KRLVFREEEENYQRLSRAHLVGGLLYTNFKKKMG-LEFTFDQGLLEESIN-AFEKKKLEKSCGTIKSHSIRSDIDWALNDVFLFMKSQLCTKYEKQFVDA KRLVFREEEENYQRLSRAHLVGGLLYRNFKNKLG-LEFTFDQGLFEESVN-AFEKKKLEKSCGTIKSHSIRSDVDWALNDVFLFMKSQLCTKYEKQFVDA KRLRFSNEMKEREKLERAHGTGSILFHNLIQKLG-LNFTWDNQLFEECVN-DFECKKLEKSKAVLANHSIRSDNDWSPNWVFLFMKSOLCTKYEKOYVDA KRLRFSEEFLERAKLKDSYSVGNLLYQNLKEKLS-LSFSWDQGLLDECLN-DFETKKLLKSKATLANHSIRSDIDWSMDKIFLFMKSQLCTKYEKQYVDA KRLRFDNVANNYAKFKAAESRGKYLTKIFLKHVP-IKCGRDQRLLDQCRQ-EFEETKLSKSAATIGAHSQRSDSDWPLDKIFLFMKSQLCTKFEKRFTEA KRLRFSNPAKNLTKFRGATAAGKILLKNFLKFIP-IPSETFPELLSEAKR-EFOEVKLKKSEGTIAGNSGRSDPDWSWDRVFLFMKSOOCTKFEKRFCEA KRLSFSNPLVESEKLNKAWIKGSILHKEFTRLIR-VNSHFRPDLFEKALN-DFEDVRMRKSEKLIMAHAGRSDPDWDIRNFLLFMKSQLCKKAEKAFCDA KRLSFSNPITEGEKLKSAFVKGSILYKEFRKIIR-VOGDFRPDLFDKALS-DFERVRVAKSKKLIEAHAGRSDPDWDVKKFLLFMKSOLCKKAEKAFSDA KRLKFSSPSVERERFEKVRHLGNEMLDIFLDKIK - IDNKLNSEMMARSYN-EYVLKKVSKTANTIASHSSRSEPDWKLNEIFLFMKTQLCTKFEKRFSDA KRLKFSSPQIEREKFEKVRHLGSEMFELLLEKIP-LDNKNDDLMMQICVN-EYIERKVSKPAGTIKSHSGRSDCDWKLNDVFLFIKTQLCTKYEKRFSDA KRLVFKNPLSNAHDFEKAKPFGKELLNIFLRKVP-LMPNFDQRMYDESVS-EFEEKKISKNAAMIGAHHDRSTTDWPTNEIFLFIKSOLCTKKEKMFCDA KRIRKSNWRREKSKFEEFESQGKELLQEFISMLP-FEFKVNIKEIEDGEK-SFLEKRKLKSEKMWANHSERSDIDWKLDHAFLFMKSQYCTKEGKMFTEA KRIVMADPFRNAMKLQKVEPISAEIFNEMNKILL - LNPHVSVDR-DQVYK-EFLRKRLNKSKKLIESHSERSSDDWPIDHFFLFMKSQLCTKFEKRFVDA KRMKMSDPYSERRKLERCMPVGENLCRLFVEEYG-LKRGWOVDI -ESTER-EFLLKRVEKAKKMIEAHSERSDPDWMVNHFFLFMKTOLCTKFEKRFSDA KRLRFRNYEANRRKYKTCHGIGHQMFSVFKDTYQ-LKEIDSLPELERCEM-EFMKKRIEKSTGLIEKHAGRSDPDWPSNYLKIFLKQQTCTKMEKRGVDA KRLRFADFEKNCASFERKKKLGESIFTEFLKRADFMNFTYPPOVDETSMELDFTMKRIOKSARILEAHSYRSDADWPSNYLKIFIKNODCTKMEKRGSDA KRLRFSKPAVEARKLMDASNFSEFMLQEFLKHVP-LKKPHNQAFMDASLA-DFEEKKTSKSAATIANHAGRSCRDWLIDTGLVFMKSQHCTKFDNRFRDA KRLRFSKPLVEARKLQDAKVYGEFMLQNFPKYVP-LRKQHNKGFMDKALR-DFESKKVSKSAAIIANHAGRSCRDWLADVGLVFMKSQHCTKFDNRFRDA KRLKFSRPATEKAKLVEAMPYGKFMLKEFLKKIP-MNKSRDTKMMEOSKL -EFEEKKLSKSAATIENHSGRSCRDWLIDIGLIFSKSOLCTKFDNRFRVA KRLSFSQPSKESAKLNRAKPYGKFLVSEFLKRIP-LRGNLDPILFAKAKR-DFEEKKTSKSAAVIENHSGRSCRDWLADVGFIFMKSQFCSKWDNRFRDA KRLSFSSPAAEHAKLRRAKPFGKFLLDTFLKRVP-LNSSHDEKMMQEAVH-AFEEKKLSKSMATIENHSGRSCEDWPVDKALIFMKSQLCTKFDNRFRSA KRLKFSLPAKEKOKFMSAIPYGDTMLKVFLNKVR-LKPNFDHRLFEEARN-DFEEKKLOKSMATLENHSGRSDPDWEIEKALIFMKSOLCTKFDNRFRDA KRLKFSFPARERQKYMAAIPYGVSMLQVFLKRIK-LQSNFDHRLFEEARA-DFEEKKLQKSMATLENHSGRSDPDWSVEKALIFMKSQLCTKFDNRFRNA $* *$ :

KAGQTLACFQHLILVFFAPWCRYMEHQIRSQLPEEIYIHSNKNFDDLNEWTKKFF- -ARDICVESDYEAFDASQDEYILSFEMHLMEDMGIPRKVIDAYV KAGQTLACFQHLILVQFAPWCRYLETQIRNQLPEEIYIHSNKNFDDLNAWVKKFF - -QRDICVESDYEAFDASQDEYILSFEIHLMKDAHFPQKIIDAY I KAGQTLACFQHLILVQFAPWCRYLEAQIRNQLPEEIYIHSNKNFDDLYRWVKNFF - -QKDICVESDYEAFDVCQDEYILSFEIHLMKDAHFPQRVIDAYI KAGQTLACFQHMILVTFAPYCRYMEKQLRAQLPGEIYIHSNKNFNDLNEWVKKHA- -GDDLCVESDYEAFDASQDQYILSFELFMMRHMHIPEQIIQAY I KAGQTLACFSHLVLAKFAPYCRYMEKMLRRNLKEEIYIHSNKNFNDLNDWVVKFF - EEGEKVESDYEAFDASODHYVLAFEVCVMEDMGLPNWFINDY I KAGQTLACFPHKILVEFSPWCRYTEKVLTANLPDNYYIHQRKNFSELEDFARRFS - -NGSICVESDYTAFDVSQDHTILAFEVELLRHFGWDDRVLQSYI KAGQTLACFSHEILCHFSPWCRYMEKVFSKYCPENFYIHQRKDFDKLAEFSRKYC--KGGFCIESDYVAFDVSQDHNVLAFEVQLMEHIRIPECVISDYI KAGQTIACFAHGVLFKFSAWCRYAELKINEVMPEAFYVHSKKNFDELERWVKGNF - - IGPICVESDYEAFDASODSTILAFECLILKDVGWPHDLIEDYK KAGQTIACFAHGVLFKFSAWARYAELKMMEKMPDSFYIHSRKNFDELEKWVKMNF - - IGPICVESDYEAFDASQDATILAFEVQFLKEVGWPQDLIEDY I KAGQTLACFSHIILNRFAAPTRYVEKKISEGLGKNFYIHQKKNFDVLNDWVVANN- -FDSYCLESDYEAFDSSQDCLILAFEYELLKYLGWDQSLLDDY KAGQTLACFSHVILNRFAAPARYIEKKLSLCLPDNYYIHQKKNFDMLNAWVVRND--FSDECLESDYEAFDSSQDCLILAFEYELLKYMGWSQSLLDDYL KAGQTLACFSHLILCKFAPLNRYIEKKVTQSLPGNFYIHQKKNFDELERWVKSYN - FSGVCTESDYKAYDASQDSCTLAFEYKLLRYLAFSNSLIEDY KAGQTLACFOHIVLFRFGPMLRAIESAFLRSCGDSYYIHSGKNFFCLDSFVTKNASVFDGFSIESDYTAFDSSODHVILAFEMALLOYLGVSKEFOLDY KAGQTLACFSHKLLTRFGPAFREFEKKFTANLPPSWYIHTMKNFDQLNNWVINYV- -DQEEGTESDYEAFDRSQDAIILGLEIECLKLFGWDQDLIDDYR KAGQTLACFSHQVLARFGVPIRVAEKKLRAQLGENIYIHSGKQLDELNEWCMGYA- - KGYGTDSDYESFDRSODALILAFELHLLRFLGWSVDQVEDYV KAGQTIACFAHSVLCRFGPILRQTEKALRELLPEKLMIYSQKKYMDLDKWAKTWV- -ESMMGTDSDYEAFDRSQDEKVLDLEVEVLRFFLWPEDLIREY KAGQTIACFSHAVLCKFGPILRKTEAQLRKILPPHVMIFSQKNYEDLDKWSKDYF - NDHSGTDSDYEAFDRSQDGAILAFEICLLRHFLWPEELIEEYK KAAQAIVCFQHAVLCRLAPFVRYIERKIAEVLPEKFYIHSGKGLEELNAWVTRGR - FEGVCTESDYEAFDASQDQYILAFELHVMKYLGLPRDLIEDYK KAAQSIVCFOHAVLCRFAPYMRYIEMKLQEVLPSNFYVHSGKGLDELSEWVKKGK - -FEGICTESDYEAFDASODQYIMAFELKVMEYLGIPKDLIADYV KAAQSIVCFQHEVLCRFAPYMRYIEKKLHQALPGNFYIHSGKGLEELNEWVMRGR - FDGVCTESDYEAFDASQDQYIMAFEIEMIKYLGLPADLISDY KAGQTLACFHHSILCRFAPYMRYIEYKLQAASPSNLYIHSGKNLEDLNEWVIRNK - -FSGMSTESDYEAFDSSQDHFILSFEIEIMKHLQLPWDLIEDYV KAGQTLACFQHSVLCRFAPYMRYIESKVTEVLPKNLYIHSGKNIDDLAAWVTTSK - -FNGVCTESDYEAFDASQDHFILAFELEVMKFLGLPSDLIADY KAGQTLACFHHNVLCRLAPYIRYIEKKVFDALPRNLYIHSGKNFDDLRDWVINSN - FSGMCTESDYEAFDSSQDANILAFEVSLMNYLNLPRDLIEDYK KAGQTLACFHHDVLCRLAPYIRYIEKKVFKALPSNLYIHSARNFDDLRDWVIKNN- -FTGVCTESDYEAFDSSQDVNILAFEVSLMEYLRLPRDLIEDYK $* * *:: * * * *^{*}: *^{*}$ 
SdRV1: AYE54584 CLBV:NP 624333.1 CLBV:AFA43536.1 AVCaV:YP_008997790.1 CPrV:AKN08994.1 ACLSV:NP 040551.1 GPGV:YP_ō04732978.2 CtChV-1:YP 009103999.1 CtChV-2:YP_009103996.1 DiVA:YP_006905850.1 ObRV1:YP 009408144.1 CVA:NP_620106.1 ASGV:NP 044335.1 PVT:YP_002019748.1 PrVT:YP_009051684.1 GVA:NP 619662.1 GVE:YP_002117775.1 GCLV:YP_004936159.1 CVNV:YP 001430021.1 PhIVB:YP_001552317.1 APV1:YP 009094347.1 ASPV:NP_604464.1 CTLaV:YP__009046478.1 CRMaV:YP_007761581.1

SdRV1: AYE54584 CLBV:NP_624333.1 CLBV:AFA43536.1 AVCaV:YP 008997790.1 CPrV:AKN08994.1 ACLSV:NP 040551.1 GPGV:YP 004732978.2 CtChV-1:YP_009103999.1 CtChV-2:YP_009103996.1 DiVA:YP 006 905850.1 ObRV1:Ȳ̄_009408144.1 CVA:NP 620106.1 ASGV:NP 044335.1 PVT:YP 002019748.1

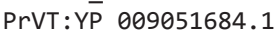
GVA:NP_- 19662.1 GVE:YP 002117775.1 GCLV:YP_004936159.1 CVNV:YP 001430021.1 PhIVB:YP 001552317.1 APV1:YP_009094347.1 ASPV:NP 604464.1 CTLaV:YP 009046478.1 CRMaV:YP_007761581.1

SdRV1: AYE54584 CLBV:NP 624333.1 CLBV:AFA43536.1 AVCaV:YP_008997790.1 CPrV:AKN08994.1 ACLSV:NP_040551.1 GPGV:YP 004732978.2 CtChV-1:YP 009103999.1 CtChV-2:YP_009103996.1 DiVA:YP 006905850.1 ObRV1:YP_009408144.1 CVA:NP_620106.1 ASGV:NP 044335.1 PVT:YP_ō02019748.1 PrVT:YP_009051684.1 GVA:NP 619662.1 GVE:YP_002117775.1 GCLV:Ȳ̄ 004936159.1 CVNV:YP_001430021.1 PhIVB:Y⿸厂二_001552317.1 APV1:YP 009094347.1 ASPV:NP_604464.1 CTLaV:YP_009046478.1 CRMaV:YP_007761581.1
DLKCTLGCKLGHFAIMRFTGEFCTFLFNTTANMAFTMCRYEWR-RGQPIAFAGDDMCALNNLPLNHD- -FDDLFDKLSLKAKVERTDCPMFCGWRLTQYG DLKCKLGCKLGHFSIMRFTGEFCTFLFNTLANMAFTMCRYEWR-RGQPIAFAGDDMCALNNLAVCHD--FDDLFELISLKAKVERTETPMFCGWRLTPYG DLKCKLGCKLGHFSIMRFTGEFCTFLFNTLANIAFTLCRYEWR-RGQPIAFAGDDMCALNNLPICHD--FDDLFELISLKAKVERTESPMFCGWRLTPYG DLKVNLGCKLGHFAIMRFTGEFSTFLFNTLANMAFTMCRYEWN-SGDPIAFAGDDMCALKNLKVTDQ- -FNNVFEKISLKAKTQITEVPMFCGWRLSRFG DLKCTLGCKLGHFAIMRFTGEFSTFLFNTLANMAFTFARYECD-HKTPIAFAGDDMCMLKACKVSDK - -FEDVLSKLSLKAKVIRTEMPMFCGWNLSRYG KMKCTLGCRLGGFAIMRFTGEFSTFLFNTLANMVFTFCRYEVP-DGTPICFAGDDMCALRNLREIDT--HEFILSKLSLKAKVNRTKVPMFCGWRLCCDG RMKTELGCKLGNFAIMRFTGEFCTFLFNTFCNMAFTFMRYQMS-GHEPICFAGDDMCALADLKESDE--YNAFFKSFSLKAKVCRTVKPLFCGWRLTKFG TLKLELGCKLGMLAIMRFTGEFGTFFFNTLANMAFTFCRYNVN-RTTPICFAGDDMCILTNAKIRNE - -MNDFIGSLKLKAKVEWKINPIFCGWILSRRG ELKVNLGCKLGNLAIMRFTGEFGTFFLNTLANMAFTFCRYNVN-RTTPICFAGDDMCILTDAKVRHD--LDEFINSLKLKAKVEWKINPIFCGWILSRYG DLKFNLGCRLGNLAVMRFTGEFGTFLFNTLANMVFTFMTYDLN-GTESICFAGDDMCCNRGIKARVDGKYDHILKRLTLKAKAVITKEPTFCGWRLTKYG DLKFNLGCRLGNLAVMRFTGEFGTFLFNTLANMVFTFMSYDLT-GKEAICFAGDDMCCNKGIRRRTDGRFDHILNRLSLKAKAVITTEPTFCGWRLTKYG YLKMHLNCKLGNLAIIRFTGEFCTFLFNTLTNMLFTFMKYDVR-KTHAICFAGDDMCANVRLPENHE - -YSSLLKKFSLKAKVDFTRSPTFCGWNLSRYG RLKLTLGCRLGSLAIMRFTGEFCTFLFNTFANMLFTQLKYKIDPRRHRILFAGDDMCSLSSLKRRRGERATRLMKSFSLTAVEEVRKFPMFCGWYLSPYG KLKLRMGCRWGAIAIMRFTGEFGTFFFNTIANIAFTCLRYNIT-RDTVIAFAGDDMYASGKLEIRKD- - REDLLAHLTLKAKVQFTEKPMFCGWYIKKMG TLKLRLGCRLGYLAIMRFTGEFGTFFLNTCCNMLFTCLRYKIN-KNTPIAFAGDDMFSPGRLEVRRD- -REFLLNRFSLKAKVNFSKEPMFCGWRMTPYG ELKLMMGCALGDLAVMRFSGEFGTFFFNTVCNMVFSCMRYHID-RNTPMCFAGDDMYSPGILRVKKD- - YEATLDOLTLKAKVHISEEPLFCGWRMSPFG TLKLMMGCQLGDLAVMRFSGEFGTFFFNTMCNMAFSYLRYQLG-PYQPIAFAGDDMVAPGRLVVNES - -MNSVLNQLELKAKVNYSDSPLFCGWRMSPYG FIKMHLGSKLGNFAIMRFSGEASTFLFNTMANMLFTFLRYEIK-GHERICFAGDDMCANARLRHRLD--QEKFLGLLKLKAKVSFTQKPTFCGWNLCSDG YIKTHLGSKLGNFAIMRYSGEASTFLFNTMANMLFTFLRYDVK-GNEFICFAGDDMCANTKLRKVDT--HESFLSKLKLKAKVGFVNKPTFCGWNLCSDG FIKTHLGSKLGNFAIMRFSGEASTFLFNTMANMLFTFLRYEIR-GNEFICFAGDDMCASKRLPLSRA--YEGFLSKLKLKAKVFFVKSPTFCGWHLSPDG YIKTHLGSKLGNFAIMRFTGEASTFLFNTMANMLFTFLRYDLN-GSEAICFAGDDMCANRRLRVSKK - -NENFLGKIKLKAKVQFTEKPTFCGWNLCMDG FIKTHLGSKLGSFAIMRFTGEASTFLFNTMANMLFTFLRYDLN-GREAICFAGDDMCANSRLKVTNR - -FSNFLDKIKLKAKVQFTATPTFCGWGLCEHG HLKFNTHSKLGQFAVMRFTGEAGTFLFNTLANMVFTFMRYETN-GRESICFAGDDMCANKLLRKKKE - -YEHVLDRMTLKAKMQHTTEPTFCGWRLGPFG YLKFHTHSKLGQFAVMRFTGEAGTFLFNTLANMVFTFMRYEIN-GREAICFAGDDMCANKLLRKKSE - -FEHILDRMTLKAKVQHTTEPTFCGWRLGNFG

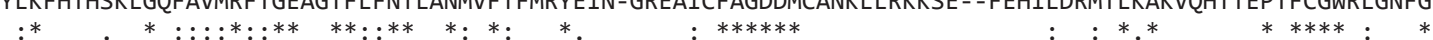

IIKEPELVYNRFQVAIEEGKVAECLENYAIEVSYAYKLSERLFEVLKSEKQLQYHQAVVRFIVKNIRKLKTNVK IVKEPELVYNRFQVAIEEGKVLECLENYAIEVSYAYSLSERLYEVLKSERQVQYHQAVVRFIVTHIDKLKTKVR IVKEPELVYNRFQIAIEEGKVMECLENYAIEVSYAYSLSERLYEVLKSERQIQYHQAVVRFIVTHIDKLKTRVK IVKEPELVYNRFMVALERGNVKDCLENYAIEVSYAYSLGERLFDILKREEQLEYHQAVVRFIVKHLGNLRTKVK IVKEPELVFNRFMVAKKRGNIDECLENYAIEVSYAYSLGEKLYEVLKREEQVEYHQAVVRFIVQRLDKLKTKVK LIKEPCLIYERLQVAIENGRLMDVIDSYFLEFSFAYKLGERLYSHLE-IEQLNYHQVLTRFFIRNKHLLRGDSRHNISEL - - . LYKEPVLVYERLKIAIEKDKLDLVIDSYFLEFCYAYKLGSWLDWVLD-EEQADYQQRLSRFFVKKRHLLKGKSLDYITHC - - - - - - - ILKLPSLVYYRLNIAKEKGNLKDCIDSYMIEAGYAYRKGAFIEELLD-EDQMSFHQLVIRSMIKSKHLMKGSSI ILKLPSLVYYRLCIALEKGNLKDCIDSYMIEAGYAYRKGAFIDELLT-EEOMNFHQLVIRRMIKAKHLMKGSSV IFKKPELVLERFLIAIEKGRLLDVIDSYYIECSYAYNLGERLFECFS-EKDFSAHYCCIRIVHKNKSLLKGLSLERYRENR - .

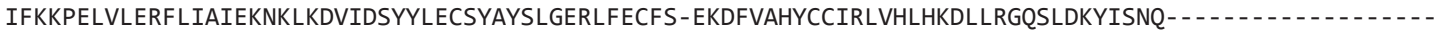
IVKKPELIAARLAVARQKGEVNLVLDSYFLEHLYAYNKGDHLFEILS-EKELEHHYNLTRFFVKNSKLLKGESKKKFMETKEIEGGLFGECDFGNDSIFK IIKSPKLLWARIKMMSERQLLKECVDNYLFEAIFAYRLGERLYTILK-EEDFEYHYLVIRFFVRNSKLLTGLSK - . IVKEPRLVLERWLIAERKKVIDQCFINYSIEVSYGYRLGEYLWEYFD- - NLEDFQAIVRLVIKKKKQLPPAIR IVKEPKLVLERFKIAEERGCFKECLINYCLEVSFAYRLGERLYDVIK- - NIQDKQALVRIVVKNKKFLPKKIRKEF IIKEPNLILDRWKIALRSGNLSLCLVNYAIEASFGYRLSEHLYDV - - NIDVDAQQELVREIVIKKHLLPKKIS IVKDPNLLLDRLEMKRAEGKLDDCIANYALEASYGYRLSDHLYDL- - NIDLDAFQELIRKIVMLKHKLPPAIA IYKKPQLVLERLCIAKETNNLACCIDNYALEVAFAYKMGERAVLRMD-EEELQSHYNCVRIILQNKNLIKSNVL IYKKPQLVLERLCIAKETNNLTSCIDNYAIEVSYAYRMSEKATMRMS-EEELDSHYNCLRIIIKNKHLMKSEAA IYKKPQLVMERMCIAKEKGNLIDCIDNYAIELSYAYKMGELALCRMD-EEETEAFYNCVRIVVKNKHLLKSDIK IFKRPQLVLERLCVAREKDNLANCLDSYAIEVGYAFALGEKILQYMD-EEALQNHYNCVRFIIKHSHLLKSSVR VFKKPDLVLERLQIARETRNLENCIDNYAIEVSCAYKMGENLNLYLT-PQEVDAHYNCVRFIVQHNHLLKSNIR IIKRPOLVOERIPIALEKGNFNECIDNYAIEVSHAYNLGDRLISIMS-EKELDAHYFCVRTFLOHKSLFSSNAL - $\ldots$ IVKRPQLVQERILIALEKGNFHECIDNYAIEVSYAYNLGERLISIMS-EKELDAHYFCVRTFLQNKKLFSSNAL $: * * * * * * * * *$

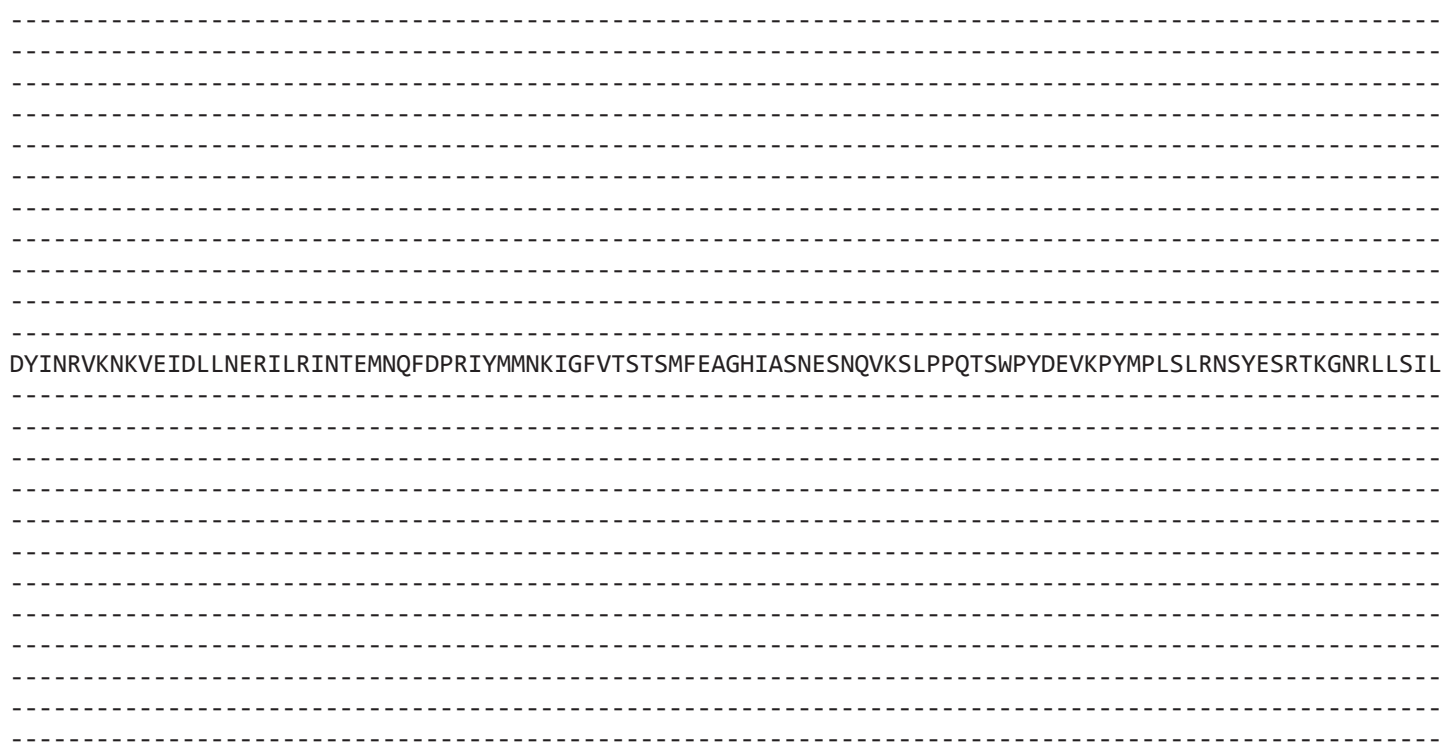


SdRV1:AYE54584 CLBV:NP 624333.1

CLBV: AFA43536.1 AVCaV:YP_008997790.1 CPrV:AKN08994.1 ACLSV:NP_040551.1 GPGV:YP_o 04732978.2 CtChV-1:YP 009103999.1 CtChV-2:YP_009103996.1 DiVA:YP_006905850.1 ObRV1:YP 009408144.1 CVA:NP_620106.1 ASGV:NP 044335.1 PVT:YP_ö02019748.1 PrVT:YP_009051684.1 GVA:NP 619662.1 GVE:YP_002117775.1 GCLV:YP 004936159.1 CVNV:YP 001430021.1 PhIVB:YP_001552317.1 APV1:YP 009094347.1 ASPV:NP 604464.1 CTLaV:YP_009046478.1 CRMaV:YP_007761581.1

SdRV1:AYE54584 CLBV:NP_624333.1 CLBV:AFĀ43536.1 AVCaV:YP 008997790.1 CPrV:AKNÖ8994.1 ACLSV:NP 040551.1 GPGV:YP $\overline{0} 04732978.2$ CtChV-1:YP_009103999.1 CtChV-2:YP_009103996.1 DiVA:YP_006905850.1 ObRV1:YP_009408144.1 CVA:NP 620106.1 ASGV:NP_044335.1 PVT:YP_002019748.1 PrVT:YP_009051684.1 GVA:NP_619662.1 GVE:YP 002117775.1 GCLV:YP_004936159.1 CVNV:YP_001430021.1 PhIVB:YP 001552317.1 APV1:YP_009094347.1 ASPV:NP_604464.1 CTLaV:YP 009046478.1 CRMaV:YP_007761581.1

\section{SdRV1:AYE54584} CLBV:NP_624333.1 CLBV:AFA43536.1 AVCaV:YP_008997790.1 CPrV:AKN08994.1 ACLSV:NP_040551.1 GPGV:YP_004732978.2 CtChV-1:YP_009103999.1 CtChV-2:YP_009103996.1 DiVA:YP 006905850.1 ObRV1:YP_009408144.1 CVA:NP_620106.1 ASGV:NP 044335.1 PVT:YP_-002019748.1 PrVT:YP_009051684.1 GVA:NP 619662.1 GVE:YP_002117775.1 GCLV:YP 004936159.1 CVNV:YP_001430021.1 PhIVB:YP_001552317.1 APV1:YP 009094347.1 ASPV:NP_604464.1 CTLaV:YP 009046478.1 CRMaV:YP_007761581.1

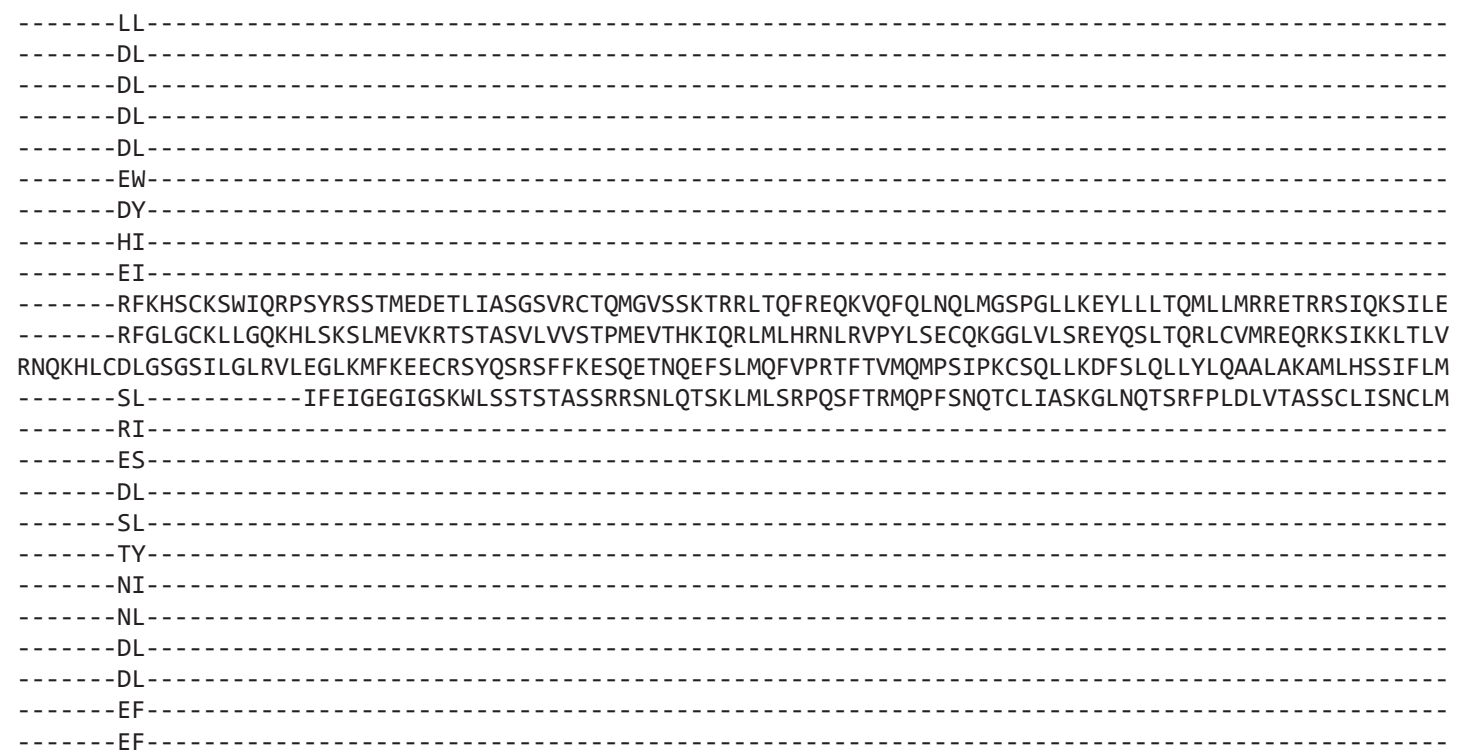

-

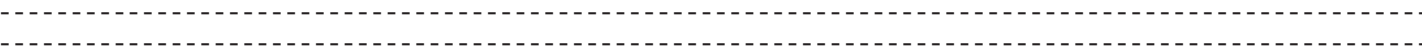

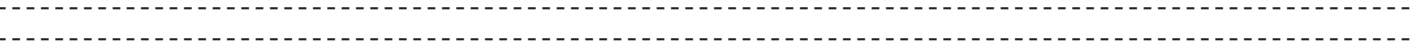

QLSSVSTSLVIMNVRCQEEDVCWLTVEEVEEVELSKPLSLIYPKDQPTSCSYQMQSLTFMMSYLTGPVKCSSSLTMLITVVVPTHLLLRLGQYIACLMSS 1857 QLLSVYTSMATMTKKPAKENACWLMEGEMMKMVSLTPSGLMFQKVQLILSLHQMLFLISMMSCLTRPVSCTFHLKVLSTEKVQDRLLLRLELSTGCQMHS 1857 KLSWRLSKKPLLSTQCFILGQSSYVLHAFSSLKSRSMAELYTLIPGFWTKMMHAKQVLVSSCKLDQPITFIGQIIQCPHMIQTCIGLLESSLNSMQSMLL 1953 TPKLIQSGRKATSTNTYTMESSWLGSKQCCQTLEAWKGESLYMMEPAWIRKEATFARIFSSLSLTVATLVSGQSTVCLPQTQIWPKGLDFVWTLIVHNMN 1753

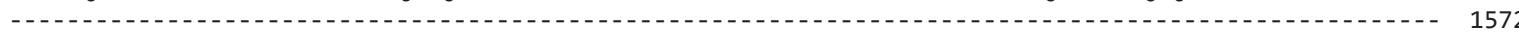

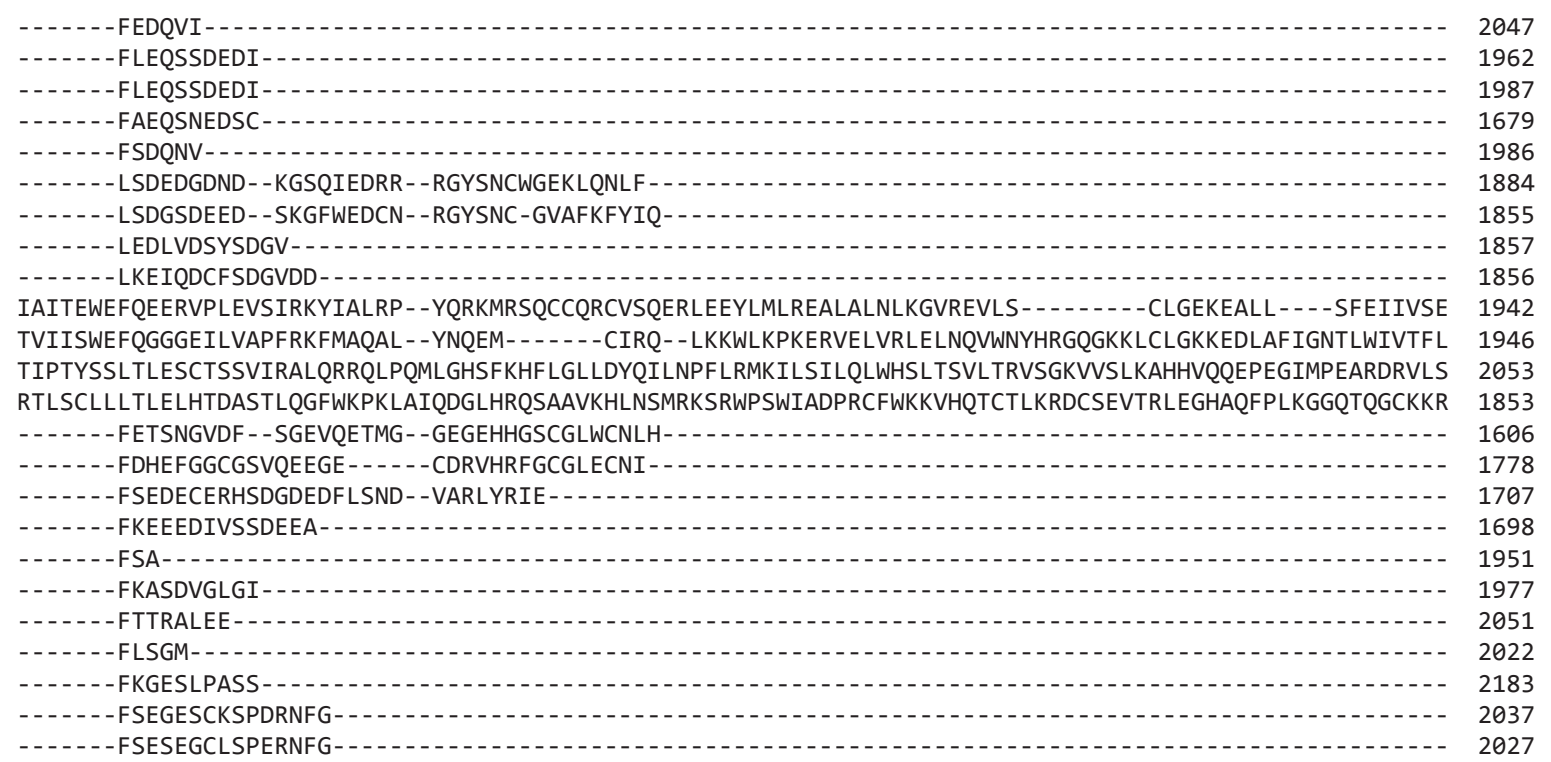


SdRV1: AYE54584

CLBV:NP_624333.1

CLBV:AFĀ43536.1

AVCaV:YP_008997790.1

CPrV:AKN08994.1

ACLSV:NP_040551.1

GPGV:YP_ō04732978.2

CtChV-1:YP 009103999.1

CtChV-2:YP_009103996.1

DiVA:YP_006̄ 905850.1

ObRV1:YP 009408144.1

CVA:NP_620106.1

ASGV:NP 044335.1

PVT:YP_002019748.1

PrVT:YP_009051684.1

GVA:NP 619662.1

GVE:YP_002117775.1

GCLV:YP_004936159.1

CVNV:YP 001430021.1

PhIVB:YP_001552317.1

APV1:YP_009094347.1

ASPV:NP 604464.1

CTLaV:YP_009046478.1

CRMaV:YP_007761581.1

SdRV1: AYE54584

CLBV:NP_624333.1

CLBV:AFA-43536.1

AVCaV:YP 008997790.1

CPrV:AKN0̄8994.1

ACLSV:NP 040551.1

GPGV:YP 004732978.2

CtChV-1:YP_009103999.1

CtChV-2:YP 009103996.1

DiVA:YP_006̄ 905850.1

ObRV1:YP_009408144.1

CVA:NP 620106.1

ASGV:NP_044335.1

PVT:YP $\overline{0} 02019748.1$

PrVT:YP_009051684.1

GVA:NP_б- 19662.1

GVE:YP 002117775.1

GCLV:YP_004936159.1

CVNV:YP_001430021.1

PhIVB:YP 001552317.1

APV1:YP_0̄09094347.1

ASPV:NP 604464.1

CTLaV:YP 009046478.1

CRMaV:YP_007761581.1

SdRV1: AYE54584

CLBV:NP 624333.1

CLBV:AFA43536.1

AVCaV:YP_008997790.1

CPrV:AKN08994.1

ACLSV:NP_040551.1

GPGV:YP_004732978.2

CtChV-1:YP 009103999.1

CtChV-2:YP_009103996.1

DiVA:YP 006 905850.1

ObRV1:YP_009408144.1

CVA:NP_620106.1

ASGV:NP 044335.1

PVT:YP_ō02019748.1

PrVT:YP 009051684.1

GVA:NP 619662.1

GVE:YP_002117775.1

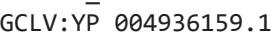

CVNV:YP_001430021.1

PhIVB:YP_001552317.1

APV1:YP 009094347.1

ASPV:NP_604464.1

CTLaV:YP_009046478.1

CRMaV:YP 007761581.1

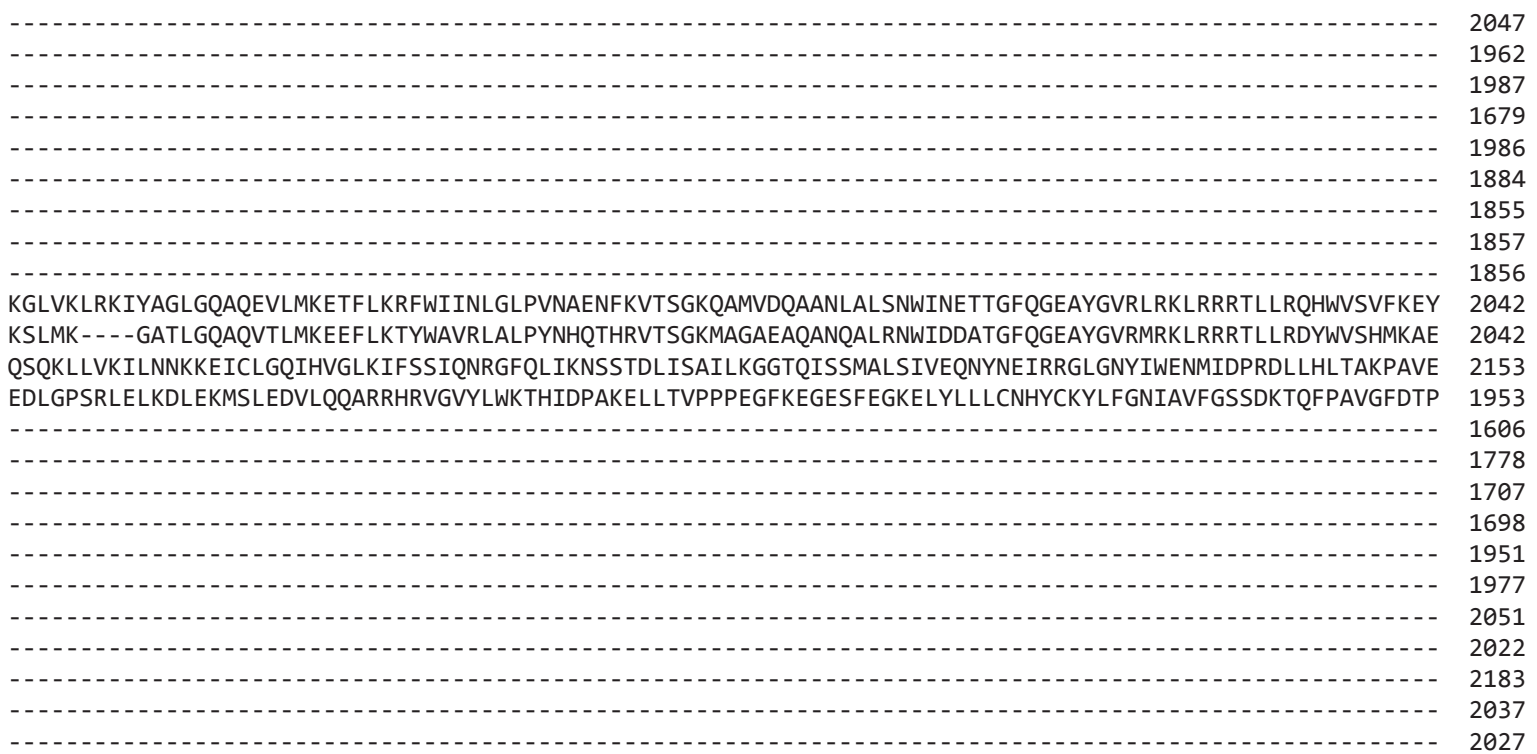

0

-

-

-

1679

1884

1855

1857

1856

VKNLGHANTPAEFTAAESEIYGRVMSDFAAYAFGIMAEEGFSPATIYN- - - EVPASYTIEYPQPVGALNVSFSPAEVSRQFKYYANSSGNSCFANITWR 2138

FQNLGHANEPQSFTAAESTLYGNIMSDFASHAFGVLAEDGFSPATVYS----SVNASYTVDYRAPVGNKTVEFSPAEVARVFKYLYQSSANPIFENMTWR 2138

ASEGVAATPAITLSENQRAVKNTIRNYYLRIMFGNLAVMGTSEQTDYPGEHLAIPRPVIENOEALTAHLPAGMSLLTFATNVKAWGVVGAEGKFAGLTFR 2253

PVHYNLTTTPKEGETDEGRKARAGSSGEKTKIWRIDLSNVVPELKTFA -

1606

1778

1707

1698

1951

1977

2051

2022

2183

2037

2027

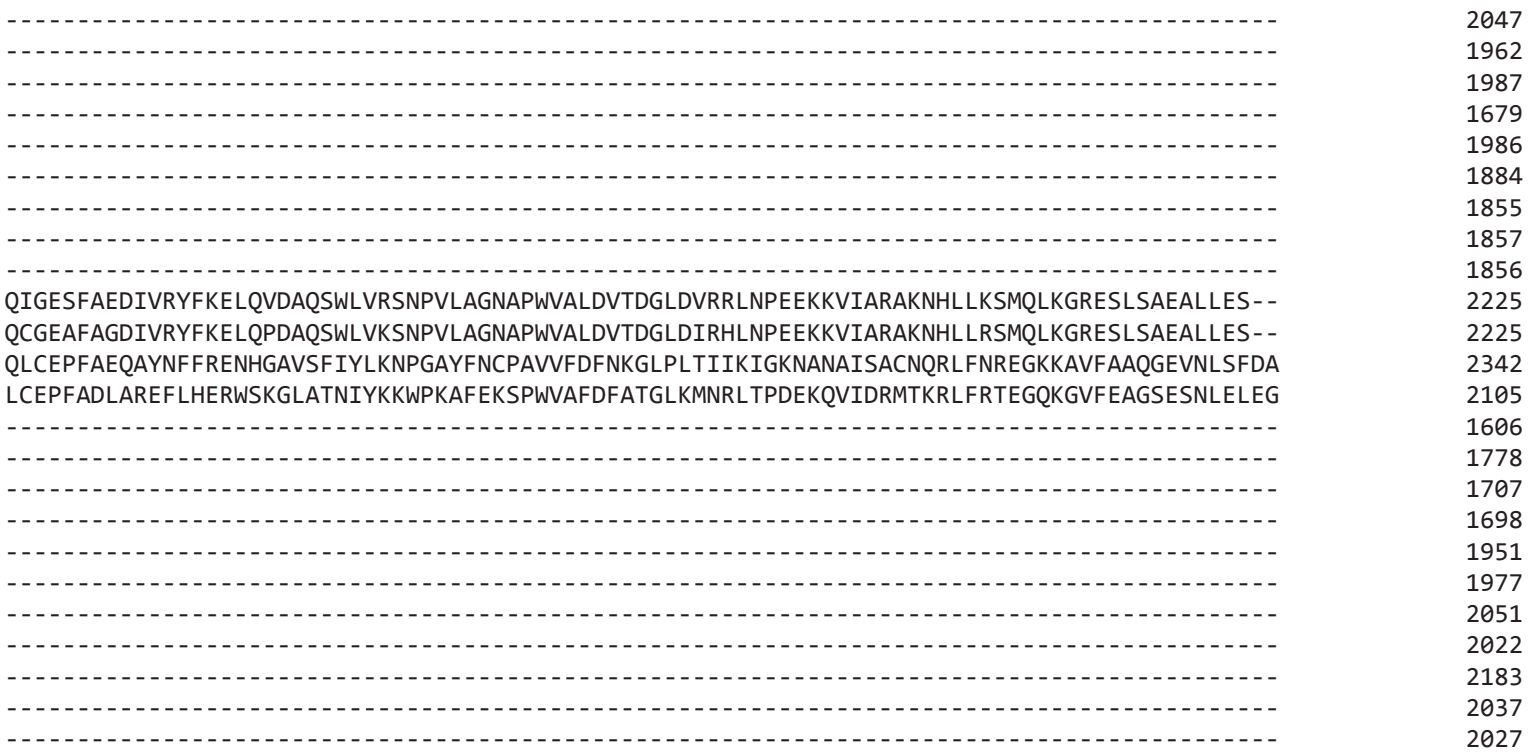


Fig. S2

Multiple sequence alignment of movement protein (MP) sequences of SdRV1 and representative members of Citrivirus and Prunevirus

SdRV1: AYE54585 CLBV:NP 624334.1 CLBV:AFA43557.1 AVCaV:YP_008997791.1 CPrV:AKN08995.1

SdRV1:AYE54585.1 CLBV:NP_624334.1 CLBV:AFA43557.1 AVCaV:YP_008997791.1 CPrV:AKNŌ8995.1

SdRV1:AYE54585.1 CLBV:NP 624334.1 CLBV:AFA43557.1 AVCaV:YP_008997791.1 CPrV:AKN08995.1

SdRV1: AYE54585.1 CLBV:NP 624334.1 CLBV:AFA43557.1 AVCaV:YP_008997791.1 CPrV:AKNŌ8995.1

SdRV1:AYE54585.1 CLBV:NP_624334.1 CLBV:AFA 43557.1 AVCaV:YP 008997791.1 CPrV:AKN0̄8995.1

MASLIRVDRLVSRIENNQSMLGSNEINNLYGSE-CARVFKDEVKLIVPGNLDGSPVVMQAPILTDERIEEIRATRIKHRGVHKRPCYLHLGFVPIAINSL MASLINVSSLVNRVKLDOSIIGSDEINKLYGSD-APLVFKDEVKMVIPGNAEGEAIKLQANILTADRLOSIRNAKVNGKEA- - - AYLHLGFVPIAIRSL MASLINVSSLVNRVKLDQSIIGSDEISKLYGSD-APLVFKDEVKMVIPGNAEGEAIKLQANILTADRLQSIRNAKVDGKEA-- - -AYLHLGFVPIAIRSL -MALINVQSLCEKLSLDESILGSSEINKLYPKDHHEFIFKDEVKLMIGGNLDGSLVSLQAPILTSERLSQIKTAKPKS- - - - - -AYLHLGFVPIVLQSL MSKAIKVSSFVNRVNLDKSLLGSNEINALYGNGFAPLVFKDEIKMTIPGNVLGKPIKVQANVLTKKRLEQIRAQKFKGKAC- - - SYIHLGVVPIAIQSL

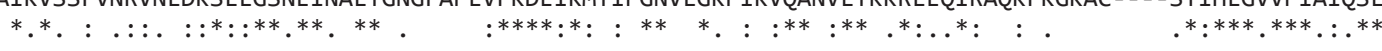

LPSGNDDVTGICALVDTSRSSYKNAVIDAFNFKWTKDEPYAAKLLTINAAIDIDCDVSVRSLOIIFKISGIDLDKERSVAAITVGLSCVPTVDMFOLPRL LPSGNEQIWGRCALVDTSRTRAETAVIDEFEFKFTKKQPFAAKLLTINAAVDINCKVSVGSIQVLLELHGVDLREERSVAAIITGLTCTPTNKMVLLHKI LPSGNEOIWGRCALVDTSRTRAETAVIDEFEFKFTKKOPFAAKLLTINAAVDINCKVSVGSIOVLLELHGVDLREERSVAAIITGLTCTPTNKMVLLHKI LPSGNDLIKGTCALIDTSRCSLSTGLIDIFKFKFTSKNPRAGKLLTINAPIDINDEVSVGSVQLLLQVEGVDLREKRSVMSITVGMSCVPTTNASLLHKL LVSGHENVWGRCSLVDLSRGSEETALIDRFKFRFTNDEPYAAKILTINAAVDINCDTSVGSLQVLLEIHGIDVRSERSVAAITVGLSCVPTNNMVMLPGL

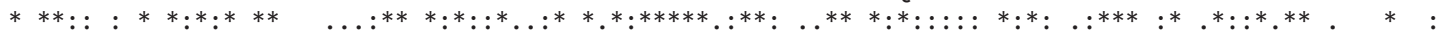

KRECPKISIMNIISSIESDVESREAFVEMFNAKKIDLLGSGKSEMLDKGKRWGF - - - - - - - FGPVVKPVSRRNLTSANRLKN- -VQIKSERC - - - - - ECDTPKWSLCNIIEQVEDEEESKKAFENMFNASSSNLIDLGQEQWLDEGKRTPLIGSLAIKGFGRKVMPVRRRNLTTRNLMKDYVSHVKSETA - - - - - ECDTPKWSLCDIIEQVEDEEESKKAFENMFNASSSNLIDLGQEQWLDEGKRVPIIGNLAIKGFGKKVMPVRRRNLTTKNLMKDYVSHVKSETA- - - - - SGERPSWNLLNVESLSSEDKESEQAFQDLFLNCKTGVVETGKIEYLKGGRKLPF - - - - - - FGKRFQPVFRRDIKTTGLLE - - - - IPTESR - - - - - KRSTPKWSLVNVFNVPEDSEAEKNAFENLFDAANPGLVDMGNDKLLETGKRMHL- - - - - WGNSLKPVYRRELSTRNLIKEQLSHVMSETAKSLKSEG

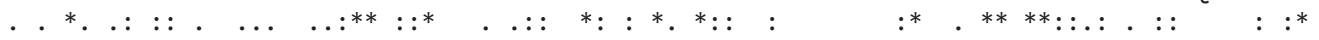
SLKRNGSLNSLKRSQSGRD- - - - SLKRSQSGGD- - - - - - EEQASEQARHSVDQQVARVPKLKTIEVTGLET SLSRSLSQR $*:$. * *

- FSRANHEEDDGLNELFTGEK- - - - - - - - -

VH- - - - - DLKDKIDKAESSTASDTGTK- - - - - - -

VH - - - - - DLKSKIDKAESSTASDTGTK -

Fig. S3

Multiple sequence alignment of coat protein (CP) sequences of SdRV1 and representative members of Citrivirus

SdRV1:AYE54586.1 MSEFKLMDRAKWPFWSSLIDGLIRFAEENDSMSEGVVEEF - -LLGSLGKNTIEEDPKKNELVYLRLCMKLISRIISNNARHKEEIRKIESDLGKRPRKEV CLBV:AFA43538.1 -MKITNDNATTINYWLAIVEPFLTSDEERN--SDDIIQKFRAVVAEHG-DTEEVDPEVFFTIFAILATKY-GRVYS---- - - --KRVE-ELNESLKVAI

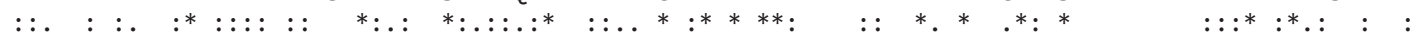

SdRV1:AYE54586.1 EVESENEKLKKIIAELHMK-ISDMEKESERSES-ETEKDRNAERRFFAGGTRS- - - - - - - - - LTNDLSCFYLGEDEFPAVTQSISTEKIATAEQIK CLBV:NP 624335.1 LAGAEAEDLRNKLKDISQRYASQLEITADREQQLESLKKKGHEQPLTGSGSSEPVHAESAHAPQLHVVNDLQQFYIPFNEYPSLTQSIGTSDIANDEHLK CLBV:AFA43538.1 LAGAEAEDLRNKLKDISQKYASQVEITADRELQIENLRKKGHEQPLAGSGSSEP - - - -AHTPQLHVVNDLQQFYIPFNEYPSLTQSIGTSDVANDEHLK

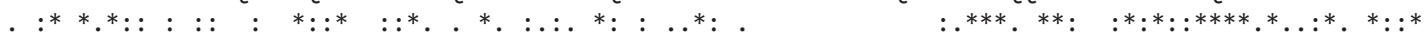

SdRV1:AYE54586.1 RVMKALNIVDEKKFSRMAFEFVISCGSKSTSSKSQYAGTFSIDNISYKRSDIAYAITGTGLTVRRFCAAYANLYWNYNIRRNQAPENWRDKNFTDETKFA CLBV:NP_624335.1 RVQLTLKITDTKVFSRTGFEFAISCGSRSTSDKDPYDGIIKISGKSHMRKDIAYAIRTSGITVRQFCAAFANLYWNFNLARNTPPENWRKKGFTEGTKFA RVQLTLKVTDTKVFSRTGFEFAISCGSRSTSDKDPYDGITKISGKSHTRKDIAYAIRTSGITVRQFCAAFANLYWINFNLARNTPPENWRKKGFTEGTKFA

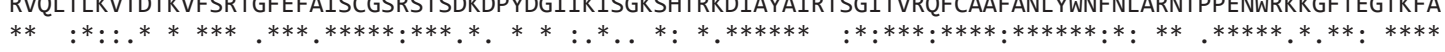

SdRV1:AYE54586.1 AFDFFYAVGSNAAIPTEPNGDVNLIRNPTKEENEANDAVKWVKIAKAKSOSMGHVTSSMFLNKGVAYNVPKSGSLSITEI CLBV:NP_624335.1 AFDFFYAVGSNAAIPTEADGSVRLIRPPTNEENEANSAMRYADIYEQNSKTAGHVTSSPLYNRGSSYESKNKAKL - - LEM CLBV:AFA43538.1 AFDFFYAVGSNAAIPTEADGSVRLIRPPTNEENEANSAMRYADIYEQNAKTAGHVTSSPLYNRGSSYESKNKAKL - - IEM 